\title{
Matter in 2+1 Dimensions at Finite Density with Chern-Simons Interactions
}

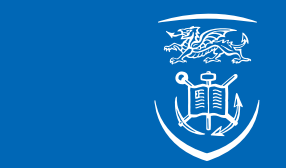

Swansea University

Prifysgol Abertawe

\author{
Stanislav Stratiev \\ Department of Physics \\ Swansea University
}

Submitted to Swansea University in fulfilment of the requirements for the degree of

Doctor of Philosophy 


\begin{abstract}
We study several matter Chern-Simons models at finite chemical potential. In the $\mathrm{SU}(\mathrm{N})$ theory we discover a colour-flavour locked Bose condensed ground state with vacuum expectation values for both the scalar and gauge fields. We identify this ground state with the non-commutative Chern-Simons description of the quantum Hall effect. We compute the quadratic spectrum and discover roton excitations. We find a self-consistent circularly symmetric ansatz for topological non-abelian vortices. We examine vortices in abelian Chern-Simons theory coupled to a relativistic scalar field with a chemical potential for particle number or $U(1)$ charge. The Gauss constraint requires chemical potential for the local symmetry to be accompanied by a constant background charge density/magnetic field. Focusing attention on power law scalar potentials $|\Phi|^{2 s}$, $s \in \mathbb{Z}$, which do not support vortex configurations in vacuum but do so at finite chemical potential, we numerically study classical vortex solutions for a large winding number $|n| \gg 1$.
\end{abstract}




\section{Declaration}

This work has not previously been accepted in substance for any degree and is not being concurrently submitted for any degree.

Signed:

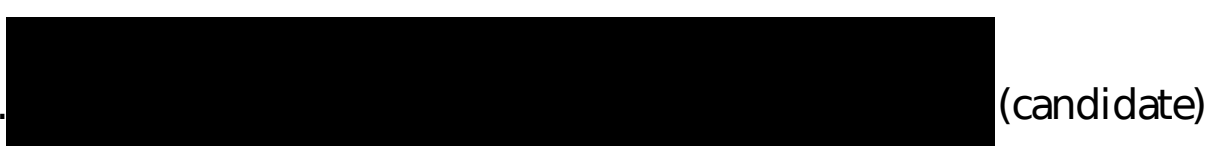

Date: $29 / 07 / 2020$

\section{Statement 1}

This thesis is the result of my own investigations, except where otherwise stated. Where correction services have been used, the extent and nature of the correction is clearly marked in a footnote(s). Other sources are acknowledged by footnotes giving explicit references. A bibliography is appended.

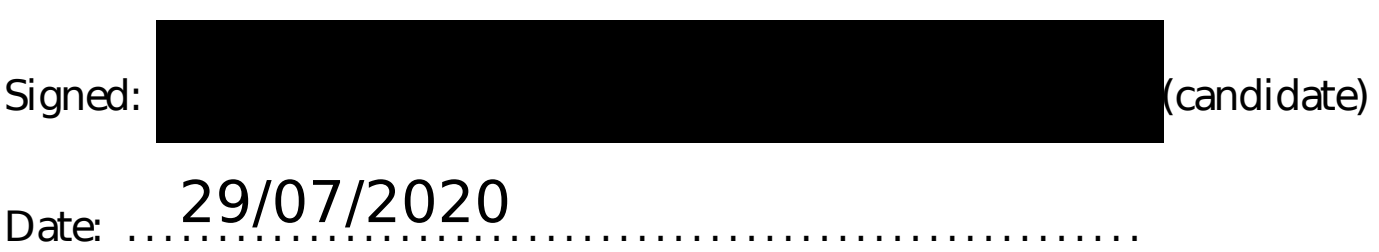

\section{Statement 2}

I hereby give consent for my thesis, if accepted, to be available for photocopying and for inter-library loan, and for the title and summary to be made available to outside organisations.

Signed:

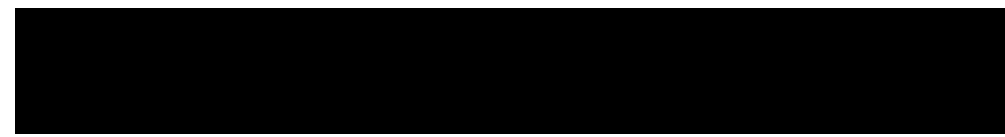

(candidate)

Date: . 29/07/2020 


\section{Contents}

List of Figures

List of Tables Xi

Introduction

1 Background 7

1.1 Chern-Simons Theory . . . . . . . . . . . . . 8

1.1.1 Chern-Simons-Maxwell theory . . . . . . . . . 18

1.1.2 Chern-Simons-matter theory . . . . . . . . 19

1.2 Statistical Physics . . . . . . . . . . . . . . 21

1.2.1 Functional integral representation of the partition function 22

1.2.2 Grand canonical ensemble . . . . . . . . . . . 25

1.3 Fractional Quantum Hall Effect . . . . . . . . . . . . . . . 28

1.3.1 Classical Hall effect . . . . . . . . . . . . . . . 29

1.3.2 Quantum Hall effect . . . . . . . . . . . . 32

1.3.3 Landau levels and the integer quantum Hall effect . . . . 32

1.3.4 Fractional quantum Hall effect . . . . . . . . . . . 36

1.3.5 Landau-Ginzburg and Chern-Simons theory . . . . . . 39

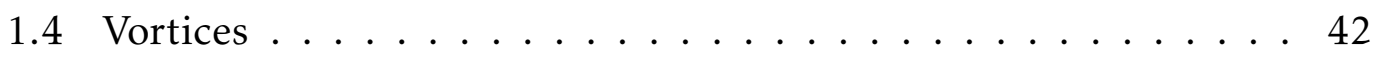

1.4.1 Global U(1) vortex . . . . . . . . . . . . 44

1.4.2 Local U(1) vortex . . . . . . . . . . . . . 45

1.4.3 Particle-vortex duality ............ 5 50

1.5 Fermi-Bose Duality . . . . . . . . . . . . . . 56

1.6 Non-Commutative Fluids . . . . . . . . . . . . . . . 58 
1.6.1 Review of non-commutative geometry . . . . . . . . 60

1.6.2 Non-commutative field theory . . . . . . . . . . 62

2 Abelian Chern-Simons Vortices at Finite Chemical Potential 65

2.1 Introduction . . . . . . . . . . . . . . 65

2.2 The Abelian Theory at Finite Chemical Potential . . . . . . . 68 68

2.2.1 Perturbative spectrum ............. 70

2.3 Vortex Equations . . . . . . . . . . . . 74

2.3.1 Qualitative features .............. 75

2.4 Vortex Energy and BPS-like Scaling . . . . . . . . . . 79

2.4.1 The quartic potential $s=2 \ldots \ldots \ldots$

2.4.2 General power law potential $(s \geq 2) \ldots \ldots 8$

2.4 .3 Positive flux vortices ............. 83

2.5 Numerical Results . . . . . . . . . . . . . . . . . 83 83

2.5.1 Quartic potential $(s=2) \ldots \ldots . \ldots 84$

2.5.2 Sextic potential $(s=3) \ldots \ldots \ldots 1$

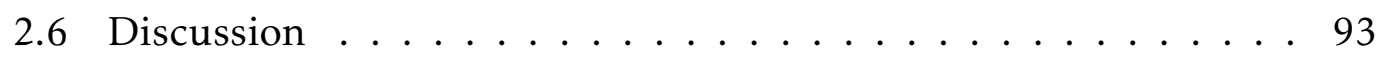

3 Roton-Phonon Excitations in Chern-Simons Matter Theory at Finite

Density

3.1 Introduction . . . . . . . . . . . . . . 95

3.2 The $S U(2)_{k}$ Theory . . . . . . . . . . . . . . 98

3.2.1 Classical ground states with $\mu_{B} \neq 0 \ldots \ldots \ldots$

3.2.2 Colour-flavour locked symmetry . . . . . . . . . . 104

3.3 Spectrum of Fluctuations . . . . . . . . . . . . . . 105

3.3.1 The $k \rightarrow \infty$ theory . . . . . . . . . . . 106

3.3 .2 Finite, large $k \ldots \ldots \ldots \ldots$. . . . . . . . . . . . . . . .

3.3.2.1 Physical states . . . . . . . . . . 108

3.3.3 Roton minimum and complete spectrum . . . . . . . . 112

3.3.4 Landau critical velocity . . . . . . . . . . . . . . 115

3.3 .5 The $U(2)_{k}$ theory $\ldots \ldots \ldots \ldots \ldots$

3.4 The $S U(N>2)$ Case . . . . . . . . . . . . . . 117

3.4.1 Vacuum configuration . . . . . . . . . . 118

3.4.2 Interpretation as quantum Hall droplet state . . . . . . . 120 
3.5 Vortices in Non-Abelian Chern-Simons Scalar Theories. . . . . . 123

$3.6 \mathrm{U}(2)$ Theory . . . . . . . . . . . . . . . . . . 124

3.7 Summary and Future Directions . . . . . . . . . . . . . 128

Conclusions

A Determinant of fluctuation matrix for $S U(2)$

References 


\section{Acknowledgements}

I'd like to thank professor Prem Kumar for his patient supervision over the past few years. 


\section{List of Figures}

1.1 This figure provides a simple example that illustrates the idea behind large gauge transformations. . . . . . . . . . . 14

1.2 This figure depicts magnetic flux attachment. . . . . . . . . . 21

1.3 This figure depicts the magnetic and scalar field profiles in the abelian Higgs model. . . . . . . . . . . . . . . 449

2.1 Dispersion relations for the abelian Chern-Simons theory in the symmetry broken phase. . . . . . . . . . . 74

2.2 This figure showcases the scalar and magnetic field profiles of vortices in the abelian Chern-Simons model at finite chemical potential and negative magnetic flux. . . . . . . . . . 84

2.3 This figure shows that the electric field profile for the vortex solutions (in the abelian Chern-Simons model at finite chemical potential and negative magnetic flux) have a ring-like profile. . . 85

2.4 This figure shows the vortex size dependence on the flux. . . . . 86

2.5 This figure shows the energy per flux for different values of the coupling parameter $\alpha \ldots \ldots \ldots \ldots 7$

2.6 This figure shows three quantities that ought to match in the BPS vortex. . . . . . . . . . . . . . . . 88

2.7 Plots of $\tilde{f}(r)^{\prime}-a(r) \tilde{f}(r)$ for 3 values of $\alpha$ and $\tilde{A}_{0}(0)$ for different values of $|n|$ and $\alpha \ldots \ldots \ldots \ldots \ldots$. . . . . . . . . . . . . . . . . .

2.8 This figure depicts the magnetic and electric field profiles for positive flux. . . . . . . . . . . . . . . 89

2.9 Magnetic field profiles comparison for $\mu=0$ and $\mu \neq 0$, in the case of $n=1 \ldots \ldots \ldots \ldots$. . . . . . . . . . . . . . . . . . . . 
2.10 Energy per flux for positive winding number $n$. . . . . . . 91

2.11 This figure shows the vortex profiles with negative winding number for quartic and sextic potentials. . . . . . . . . . 92

2.12 This figure shows the scaling of the energy of the $n$-vortex with $|n|$ and $\alpha$, with sextic potential. . . . . . . . . . 92

2.13 This figure shows value of $\tilde{A}_{0}$ at the origin for different values of $\alpha$, as a function of $|n|$, with the sextic potential. . . . . . 93

3.1 This figure shows the effective potential (free energy density) as a function of the VEV $v$ for $\mu_{B}=1, m=0.5$ and $g_{4}=0 . \ldots 104$

3.2 This figure shows the value of the speed of sound as a function of the dimensionless parameter $\tilde{\mu}=\pi\left|\frac{\mu_{B}}{g_{4} k}\right|$ for the massless theory.110

3.3 This figure shows how the inclusion of a Chern-Simons term causes a level-splitting. . . . . . . . . . . . . . 111

3.4 This figure shows the position and heigh of the roton maximum, in addition to the mass of the lowest state for large values of $k$. . 112

3.5 This figure shows two plots. One depicts the spectrum in the massive case, whereas the other shows the free scalar theory coupled to Chern-Simons. . . . . . . . . . . . . . . 114

3.6 This figure shows the Landau critical velocity as a function of the dimensionless parameter $\pi \frac{\mu_{B}}{g_{4} k}$ in the theory with $m=0$. . . 116

3.7 This figure shows the semi-classical spectrum of the $U(2) \simeq S U(2) \times$ $U(1)$ theory. . . . . . . . . . . . . . . 117 


\section{List of Tables}

1.1 This table shows the matching of the phases of the XY model and the abelian Higgs model. . . . . . . . . . . 53 


\section{Introduction}

On the second day of my graduate studies, the Nobel prize in physics was awarded to D. Thouless, M. Kosterlitz \& D. Haldane for the discovery of topological phase transitions? At the time I thought topology was a subject about open neighbourhoods, Hausdorff spaces and counting holes on mugs and doughnuts. I could not fathom how these ideas could have anything to do with the description of the real world. I had to understand how these concepts were connected. This thesis follows my path of exploring the mystery of topological physics from the perspective of high energy physics and quantum field theory (QFT).

Today, topology has become ubiquitous in the study of physics. It provides us with new models that give us insight into nature. In addition, topology provides the stability for new kinds of excitations in models whose initial purpose had more to do with the study of particles (for example, topological degrees of freedom were discovered in the Georgi-Glashow model [1] by 't Hooft and Polyakov in 1974 [2, 3]). Even very deep ideas such as the understanding of confinement and the attempts to study strongly correlated gauge theories seem to be intimately related to the study of continuous deformations. There are hints that confinement might be related to instanton condensation [4]. Specifically relevant to this work, we find that relating strongly and weakly coupled theories through dualities maps the conserved current of a theory to the topological current of its dual. It was our hope that we could shed some more light on the details of these dualities at finite matter density and, for this reason, this thesis has been centered around the study of the grand canonical ensemble of Chern-Simons matter theories, specifically scalar field theories.

\footnotetext{
${ }^{1}$ https://www.nobelprize.org/prizes/physics/2016/summary/
} 
Before we proceed to list the results of this thesis, we take the time to track down the history of the subject of dualities. In 1941, Kramers and Wannier [5] found that the two dimensional Ising model is dual to itself, under a transformation of the coupling, which allowed them to compute the critical point of the theory, several years before it was solved exactly by Onsager [6]. Then, in 1975, Coleman [7] showed an equivalence between the sine-Gordon model [8] and the massive Thirring model [9] in two space-time dimensions, establishing the first known Fermi-Bose duality. Later on, this was generalised by Witten [10] to non-abelian theories in $1+1$ dimensions. Also concerning a 1+1 dimensional system, level-rank duality relates two different Wess-ZuminoWitten models with different gauge groups and levels of their Kac-Moody algebras [11, 12]. Adding an extra dimension, Naculich, Schnitzer, Riggs \& Mlawer [13, 14] showed that the same type of duality applies to pure Chern-Simons theory, a topological quantum field theory (TQFT) in 2+1 dimensions.

Another example of a duality dates further back to Maxwell's equations in free space, which possess the property of being invariant under an exchange of electric and magnetic fields

$$
E \rightarrow B, \quad B \rightarrow-E,
$$

where $\boldsymbol{E}$ and $\boldsymbol{B}$ are the electric and magnetic fields, respectively. This duality can be preserved in the presence of sources if we were to also allow for sources of magnetic charge. Dirac [15] took the idea of a magnetic source (magnetic monopole) seriously and showed that if one allows for a singularity (we shall see later that a singularity is actually not necessary) in the vector potential, a configuration with the property of a magnetic monopole can exist. Further still, he proved that electric charge would be quantised if such a configuration exists

$$
e q=2 \pi \hbar n
$$

where $e$ is the electric charge, $q$ is the magnetic charge of the hypothetical monopole and $n \in \mathbb{Z}$. The idea that duality transforms elementary particles into non-perturbative objects (here the point electron turns into a Dirac string) 
often plays a prominent role in the dual description of a theory. This is precisely what happens in the non-abelian generalisation of electromagnetic duality.

In 1977, Olive $\mathcal{E}$ Montonen [16] proposed that, in the Georgi-Glashow model, electromagnetic duality is preserved in the full quantum theory and that the elementary excitations are exchanged with the monopoles discovered by ' $t$ Hooft and Polyakov [2, 3], and vice-versa. Today, this equivalence is known as S-duality, also referred to as strong-weak duality. Many checks for this conjecture have been made for the supersymmetric (SUSY) version of this theory [17-20]. In 1994, Seiberg [21, 22] and Seiberg $\mathcal{E}$ Witten [23] solved the $\mathcal{N}=1$ and $\mathcal{N}=2$ Super Yang-Mills (SYM) theories exactly, putting the duality on solid ground for supersymmetric theories in $3+1$ dimensions. This version of S-duality became known as Seiberg duality.

It is a natural question to ask what happens to Seiberg duality in a different number of dimensions, particularly of interest to us, in this work, would be the case of two spatial dimensions and one time dimension. The first cases of dualities that resemble Seiberg duality in $2+1$ dimensions were found by Aharony and Karch [24, 25]. These were later generalised to include a ChernSimons term by Giveon E Kutasov [26], generalising the level-rank dualities, that we mentioned earlier, to include supersymmetric matter.

Dualities also played a major role in the second superstring revolution, when several of the distinct self-consistent string theories were shown to be dual to each other [27-30] and possibly dual to an 11-dimensional theory, known as M-theory [31].

Another example of duality that comes from string theory is one inspired by the study of black holes and the holographic principle [32, 33]. This is the well-studied AdS/CFT correspondence [34, 35], which states that one can identify operators on the Anti-de Sitter (AdS) space with operators on the boundary of the AdS space, which is a conformal field theory (CFT). A special duality of this type involves a peculiar $3+1$ dimensional gravitational theory on an $\mathrm{AdS}_{4}$ background with a matter content comprising of an infinite tower of higher $(>2)$ spin fields. This higher spin theory is known as Vasiliev's gravity, named after its inventor [36]. It turns out that this theory is dual to 
free $O(N)$ and $S U(N)$ scalar and fermion theories in $2+1$ dimensions in the large $N$ limit [37, 38]. This connection between bosonic and fermionic theories through holographic duality, was the main motivation for researchers to consider the possibility that there is a duality connecting these bosonic and fermionic theories directly.

The existence of such a duality was first speculated by Minwalla et al. [39]. The statement of the duality was made precise by Aharony [40]. It has been shown that one can reach this duality through a renormalisation group (RG) flow from one of the supersymmetric dualities we discussed above [41].

The first novelty that we stumbled upon in the work outlined in this thesis, was a ground state that had been overlooked since it only manifests itself in the finite chemical potential regime. It is a ground state with non-zero expectation value for the gauge fields, seemingly breaking rotational symmetry. In reality, colour-flavour locking remedies this and the rotational symmetry is preserved. In order to confirm that the rotational symmetry is preserved, we computed the quadratic spectrum of the theory, which showed no signs of rotational asymmetry. We discovered further, owing to Goldstone's theorem and the broken $U(1)_{B}$ global symmetry, that the long wave-length dynamics of the $S U(2)$ theory behaved as a superfluid and predicts the existence of a roton excitation. What was even more surprising is that this non-trivial ground state exists in the zero coupling free scalar theory coupled to Chern-Simons. This is peculiar since we generally expect there to be a potential that drives the symmetry breaking.

Correlation functions for the scalar Chern-Simons theory at chemical potential $\mu=0$ have been computed exactly in the large $N$ limit [42]. Since the $\mu=0$ results cannot be extrapolated to finite $\mu$, we would need to take the ground state discussed in the previous paragraph into account. Further, Jain et al. [43] have computed the partition function for all values of temperature and chemical potential. However, this result again hinges on the assumption that the ground state of the system is trivial. We show this is not the case. A natural question that arises is, what do the correlation functions look like in the presence of this new condensate at large N? Answering this question 
was the ultimate aim that we had in commencing this work, i.e. solving this theory in the large $N$ limit. In order to pursue this direction, we set ourselves the more conservative goal of computing the quadratic spectrum in the $S U(N)$ theory, for general $N$. The ground state in the $S U(N)$ theory has the same algebraic structure as the non-commutative Chern-Simons theory used as a model for the fractional quantum Hall effect (FQHE). The approach to diagonalising the large $N$ spectrum of fluctuations that we outlined involves translating the problem to a non-commutative Chern-Simons theory and using the solutions of that model to arrive at an exact result.

Aside from studying the ground state and the fundamental excitations of finite density Chern-Simons models, we have also explored the existence and properties of solitons (vortices) in these models. Our main results in this line are the discovery and study of an approximate numerical Bogomol'nyi, Prasad E Sommerfeld (BPS) state in the abelian theory. Additionally, we find a selfconsistent vortex ansatz for the $S U(2)$ and $U(2)$ theories. We have left the numerical study of the resulting equations for future work.

This thesis is structured in the following way:

In Chapter (11), we cover the necessary background required to understand the rest of the thesis and how it fits in the wider field of research. We present a condensed introduction to the topics of Chern-Simons field theory [44-47], the quantum Hall effect [48-55], vortices [56-69], particle-vortex duality [7077], Fermi-Bose duality [26, 39-441, 43, 78-81], the statistical grand canonical ensemble in quantum field theory and non-commutative field theory [82-93].

Chapter (2) presents the analytical and numerical study of abelian vortex solutions interpolating between a symmetric and asymmetric phase, where the symmetry breaking is chemical potential driven.

Chapter (3) showcases the derivation of the peculiar ground state, which we mentioned earlier, and the spectrum of fluctuations is analysed in the $S U(2)$ theory. This is followed by the $S U(N)$ ground state and the observation that it resembles non-commutative Chern-Simons theory. At the end of the chapter, we switch gears back to vortices, except this time we consider the possibility of topological solutions in the non-abelian theory. We show that 
there is a self-consistent circularly symmetric ansatz for a global vortex in the $S U(2)$ theory and a local vortex in the $U(2)$ theory. 


\section{Chapter 1}

\section{Background}

This chapter provides the necessary background information and technical details required to understand the main results of this thesis and their implications to related fields of physics. This background material includes both the theoretical framework used in this thesis and associated real-world phenomena. As the title hints, in this text, we will concern ourselves with the study of $2+1$ dimensional systems at finite density. We will approach the study of these systems through the framework of quantum field theory (QFT).

There are two important aspects of QFT that will play a major role for us. One of them is the relation between the grand canonical statistical partition function and QFT. The other aspect is the distinct way in which the gauge principle manifests itself in $2+1$ dimensional theories. The latter is addressed in Section (1.1), where we review the basics of Chern-Simons theory (CS). The former is discussed in Subsection 1.2.2, where we will spend time reminding ourselves of some basic statistical mechanics.

After we have covered these, we will spend some time looking at the problems of the fractional and integer quantum Hall effects (FQHE/IQHE) in Section (1.3) and emphasise their relation to Chern-Simons matter theories. This will help us understand the applications and motivate the study of the models we are concerned with in this text.

Then, we shall continue by reviewing the properties of vortices in Section (1.4). Specifically, we look at vortices in the abelian Higgs model, pure abelian 


\section{BACKGROUND}

Chern-Simons theory with scalar matter and the mixed case where both classical Maxwell dynamics and the topological Chern-Simons term play a role. We emphasise the importance of a Bogomol'nyi-Prasad-Sommerfield (BPS) [68, 69] bound for these systems. Finally, we discuss some subtleties relating to nonabelian vortices.

We continue by addressing Fermi-Bose and particle-vortex dualities in Section (1.5), which provided the primary motivation for this work. We outline important aspects of the dualities in order to highlight where our work fits in that context.

Finally, we include a section concerning the physics of non-commutative field theory and its relation to the theory of fluids. In particular, we include the description of the quantum Hall fluid in terms of a 'fuzzy' underlying space, in which the coordinates do not commute. We shall see that this description is a Chern-Simons theory and it seems to arise naturally out of the ground state in the finite density non-abelian model, studied in Chapter (3).

\subsection{Chern-Simons Theory}

Chern-Simons theory is a very unusual type of QFT both in its origin and its applications. It is a testament to the power and universality of mathematics, first conceived of as an abstract tool in the theory of differential operators and their connections to topology [94]. The theory has grown to encompass many facets of modern physics and mathematics, from the computation of knot invariants [47] to the prediction of non-abelian anyons [95], which are the ingredients necessary for performing a topological quantum computation [96]. This is currently one of the most promising approaches to fault tolerant quantum computation [97], due to the topological stability of the qubits. CS theory is an example of a topological quantum field theory (TQFT) of Schwarz type [98], which means that it is independent of the metric and so any observable is related to a topological invariant. The Einstein-Hilbert action in $2+1$ dimensions can be written as the CS action [99]. This means that studying CS theory also leads to insight into three dimensional gravity [100-102]. CS theory also provides a field theoretic explanation for the quantum Hall effect 
and anyonic physics [48, 103], has been instrumental in the classification of rational 2 dimensional conformal field theories (CFTs) [104], creates a bridge between theoretical physics and knot theory [47] and, recently, it has uncovered an equivalence between two types of matter, previously thought to have been fundamentally different - namely, Fermi-Bose duality [26, 39, 40, 42, 105]. These are among the most noteworthy applications of CS theory. Therefore, in this section we justly take the time to review essential aspects of CS theory. Most of the results seen in this section can also be found in the excellent review by Dunne [46].

In the study of fundamental physics, we should assume that any term in the Lagrangian that is not forbidden by a basic principle (e.g. a symmetry or gauge-invariance) should be included in order to account for all aspects of the system at hand. From gauge theory we are familiar with the idea of introducing a gauge field to render a Lagrangian gauge invariant and then including a gauge-invariant Yang-Mills term that is responsible for the dynamics of the gauge potential. Modulo subtleties, this is true in any number of dimensions. Taking these subtleties into account, it turns out that in $2+1$ dimensions, the Yang-Mills term is not the only gauge invariant term one can include. One can write down a term in the action, which is the integral of a three-form, depending solely on the gauge field $A_{\mu}$. This term, whilst not manifestly gauge invariant, leaves the partition function unchanged under gauge transformations. This three-form is the Chern-Simons Lagrangian, which we write down here

$$
\mathcal{L}_{C S}=\kappa \epsilon^{\mu \nu \rho} \operatorname{Tr}\left[A_{\mu} \partial_{\nu} A_{\rho}+\frac{2}{3} A_{\mu} A_{v} A_{\rho}\right] .
$$

Here the coupling constant $t^{1} \mathcal{K}$ is conventionally referred to as the Chern-Simons level. This name hints at the discrete nature of this quantity and we shall shortly see that, under certain conditions, one can derive a quantisation constraint for $\kappa$. The gauge field $A_{\mu}$ takes values in a finite dimensional representation $\mathcal{R}$ of the (semi-simple) gauge Lie algebra $\mathscr{G}$. The $\operatorname{Tr}$ operation is the

\footnotetext{
${ }^{1}$ Why we are referring to it as a coupling constant will become apparent when we look at the inclusion of matter in CS models
} 
standard matrix trace for a $\operatorname{dim}(\mathcal{R}) \times \operatorname{dim}(\mathcal{R})$ matrix. In an abelian theory, the gauge fields $A_{\mu}$ commute, and so the trilinear term in 1.1 vanishes due to the antisymmetry of the $\epsilon^{\mu \nu \rho}$ tensor density. In the non-abelian case (just as in Yang-Mills theory) we write

$$
A_{\mu}=A_{\mu}^{a} T^{a}
$$

where the $T^{a}$ are the generators of $\mathfrak{b}$ (for $a=1, \ldots, \operatorname{dim}(\mathbb{b})$ ), satisfying the commutation relations

$$
\left[T^{a}, T^{b}\right]=f^{a b c} T^{c}
$$

and the normalisation

$$
\operatorname{Tr}\left(T^{a} T^{b}\right)=-\frac{1}{2} \delta^{a b}
$$

We can define the field strength tensor in the usual way for a non-abelian gauge theory

$$
F_{\mu \nu}=\partial_{\mu} A_{v}-\partial_{\nu} A_{\mu}+\left[A_{\mu}, A_{\nu}\right]
$$

$F_{\mu \nu}$ transforms under a gauge transformation in such a way that any expression that is the trace of a string of $F_{\mu \nu}$ is gauge invariant. More precisely, under a gauge transformation $g \in G=\exp [\overleftarrow{b}]$,

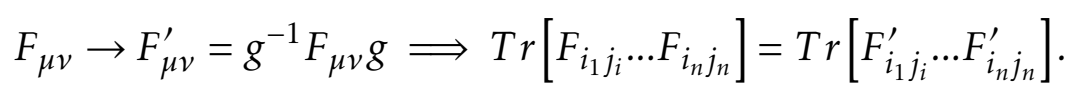

Since the Lagrangian 1.1 is written in terms of $A_{\mu}$ as opposed to $F_{\mu \nu}$, gauge invariance is not manifest.

Before we delve into the details of the gauge invariance of this Lagrangian, it is helpful to venture into a quick digression about topology. Topological invariants are such properties of spaces (and hence of field configurations defined on those spaces) that do not change under continuous transformations. And since most phenomena in the real world are continuous, we should expect 
that these invariants are important tools that we can use to better understand the physical world. Indeed, we shall see that these expectations are justified.

One way topologists use to classify spaces is through homotopy groups. An example is the first homotopy group $\pi_{1}$ or the fundamental group. This group is an indicator of the "connectivity" of a manifold. Its definition has to do with whether a given loop can be contracted to a point. More precisely, one can define a group at each point on a manifold, whose elements are equivalency classes of loops that start and end at that point and are continuously deformable into each other. For example, if all loops on a given space can be contracted to a point, then the fundamental group $\pi_{1}$ is trivial, since all elements of the group are the identity. The group operation on these loops is such that we create a new loop, $g_{1} \circ g_{2}$, from $g_{1}$ and $g_{2}$ by going around $g_{1}$ first and then going around $g_{2}$. It turns out that loops that encircle a singular point (or a hole in the space) $n$ times are not continuously deformable into loops that encircle said point $n^{\prime}$ times, if $n \neq n^{\prime}$. This is an intuitive way of understanding the fact that for each hole in the space-time manifold, the fundamental group acquires a Cartesian factor of $\mathbb{Z}$. This means that loops can wind around singular points arbitrarily many integer number of times and the number of loops around one singular point is independent of the number around another such singular point.

Such a classification is not restricted to loops but can also be applied to higher dimensional spheres. For example, the classes of mappings from the 3 -sphere to three dimensional Euclidean space-time are classified by $\pi_{3}$. If $\pi_{3}$ is non-trivial, this tells us that not all gauge transformations are connected to the identity. Importantly, for the purposes of theoretical physics, we shall use the fact that $\pi_{3}(S U(N))=\mathbb{Z}$ for $N \geq 2$ is non-trivial [106]. We shall now see how this fact plays a role in the gauge invariance of Chern-Simons theory.

To convince ourselves of the gauge invariance, let us perform a gauge transformation

$$
A_{\mu} \rightarrow A_{\mu}^{\prime} \equiv g^{-1} A_{\mu} g+g^{-1} \partial_{\mu} g, \quad g \in \mathcal{G}
$$




\section{BACKGROUND}

where $\mathcal{G}$ is a compact semi-simple Lie group. Under this transformation, the Lagrangian changes in the following way

$$
\mathcal{L}_{\mathrm{CS}} \rightarrow \mathcal{L}_{\mathrm{CS}}-\kappa \epsilon^{\mu \nu \rho} \partial_{\mu} \operatorname{Tr}\left[\partial_{\nu} g g^{-1} A_{\rho}\right]-\frac{\kappa}{3} \epsilon^{\mu \nu \rho} \operatorname{Tr}\left[g^{-1} \partial_{\mu} g g^{-1} \partial_{\nu} g g^{-1} \partial_{\rho} g\right] .
$$

The second term is just a boundary term. If we are either considering manifolds with no boundary or we insist that the gauge transformation approaches 0 at $\infty$, this term vanishes. This will be the case in this thesis. For an $S U(2)$ transformation, the third term turns out to be proportional to the winding number density of the gauge transformation, whose integral is an integer $n$ homotopy invariant

$$
w(g)=\frac{1}{24 \pi^{2}} \epsilon^{\mu v \rho} \operatorname{Tr}\left[g^{-1} \partial_{\mu} g g^{-1} \partial_{\nu} g g^{-1} \partial_{\rho} g\right], \quad \int d^{3} x w(g(x))=n \in \mathbb{Z} .
$$

The integral of $w(g)$ is also known as the Brouwer degree of a mapping. Here, the factor of $24 \pi^{2}$ is proportional to the volume of the $\mathrm{SU}(2)$ group and depends only on the choice of gauge group and the convention we have chosen for the Killing form (the trace of the product of two generators).

For the case of a mapping between one $n$-sphere and another $n$-sphere (which is indeed the case for an $S U(2)$ gauge transformation on $S^{3}$ ), we are guaranteed that this quantity is a homotopy invariant by Hopf's theorem [107]. Hopf's theorem states that two mappings $f, g: S^{n} \rightarrow S^{n}$ are homotopic if and only if they have the same winding number.

Another way of seeing that $w(g)$ is a homotopy invariant is by varying $g \rightarrow$ $g+\delta g$ and showing that the integral of the first order variation of $w(g)$, which we define to be $\delta w(g)$, vanishes. Using the fact that $(g+\delta g)^{-1}=g^{-1}-g^{-1} \delta g g^{-1}$ to first order, and the cyclicity of the trace, we can show that

$$
\int d^{3} x \delta w(g)=\frac{1}{24 \pi^{2}} \epsilon^{\mu \nu \rho} \int d^{3} x 3 \partial_{\mu} \operatorname{Tr}\left[\left(g^{-1} \delta g\right) g^{-1} \partial_{\nu} g g^{-1} \partial_{\rho} g\right] .
$$

Using Stokes' theorem and requiring that the variation $\delta g$ vanishes at the boundary (or equivalently, considering a manifold without a boundary, which 
is the case for $S^{n}$ ), we see that

$$
\int d^{3} x \delta w(g)=0
$$

Thus we see that the winding number is unaffected by small variations of the mapping.

Gauge transformations with a non-zero winding number are called large gauge transformations. Another way of saying this is that they are mappings that are not continuously connected to the identity transformation.

Here we take a break from Chern-Simons theory to give a simple example to illustrate how these gauge transformations are different. Suppose we have a complex scalar field $\varphi$ defined on $S^{1}$

$$
\varphi: S^{1} \rightarrow \mathbb{C}
$$

such that $\varphi=f(\theta) e^{i \alpha(\theta)}$, where $f \in \mathbb{R}$ and $\theta$ describes the position on the $S^{1}$. Here the $e^{i \alpha(\theta)}$ can be absorbed into a $U(1)$ gauge transformation. Below (Fig 1.1 we have pictorially represented the phase of the scalar $\varphi$ at a point on the manifold (in this case $S^{1}$ ) as a red arrow pointing in a direction on the plane. In Fig $(1.1)$ ) we have $\alpha(\theta)=\frac{\pi}{2}$. It is not hard to convince ourselves that we can transform this configuration to $\alpha(\theta)=0$ by making the same infinitesimal changes at every point on $S^{1}$. Thus $\alpha(\theta)=\frac{\pi}{2}$ is an example of the familiar kind of "small gauge transformation". One can go from $(1.1 \mathrm{a})$ to $(1.1 \mathrm{~b})$ by setting $\alpha=\theta-\pi$. However, in this case we see that there is no way to make small transformations that are continuous. Configurations $(1.1 \mathrm{~b})$ and $(1.1 \mathrm{k})$ are said to be in a different homotopy class from $(1.1 \mathrm{a})$, but they are in the same gauge equivalency class as $1.1 \mathrm{a})$.

As a final remark to counter the point that "We do not live on a circle", I shall say that when studying a non-singular, circularly symmetric, planar setup, we would require boundary conditions which identify the point at infinity with the same gauge field configuration. It turns out that the point at infinity is an $S^{1}$, so inevitably abelian gauge transformations would be separated into distinct homotopy classes. A discussion of this fact can be found in 


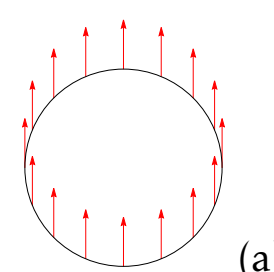

(a)

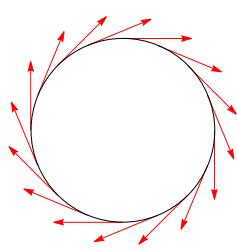

(b)

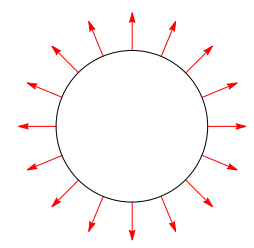

(c)

Figure 1.1: This figure provides a simple example that illustrates the idea behind large gauge transformations. The phase of a scalar field $(\in(0,2 \pi))$ has been pictorially represented by arrows pointing in some direction on the plane. The three configurations depicted can all be transformed into each other via a gauge transformation. Transforming (a) into either (b) or (c) involves a gauge transformation that is not connected to the identity, i.e. it cannot be represented as a sum of infinitesimal, continuous transformations and, for this reason, it is called a large gauge transformation. So we say that (a) is topologically distinct from the rest, yet they are all gauge equivalent configurations.

a review of Yang-Mills theory by Jackiw [108].

This above passage illustrates the essence of large gauge transformations in the simplest possible case, where we have a non-trivial homotopy group. The situation is precisely the same with more complicated homotopy groups but they just happen to be more difficult to visualise.

Going back to the Lagrangian transformation law, we pick $g$, such that $\int d^{3} x w(g)=n$. Therefore the action $S_{\mathrm{CS}}=\int d^{3} x \mathcal{L}_{\mathrm{CS}}$ transforms as

$$
S_{\mathrm{CS}} \rightarrow S_{\mathrm{CS}}+8 \pi^{2} \kappa n
$$

Here we see that the action is not gauge invariant! However, keep in mind that what plays a role in the path integral is $e^{i S}$, which transforms as

$$
e^{i S_{C S}} \rightarrow e^{i 8 \pi^{2} \kappa n} e^{i S_{\mathrm{CS}}}
$$

This implies that if we set $\kappa=\frac{k}{4 \pi}$, where $k \in \mathbb{Z}$, we have restored gauge invariance, which means that we ought to include this term in the study of planar physics. This argument for the quantisation of the CS level is due to Deser, Jackiw E Templeton [45].

The previous discussion can be easily generalised to gauge groups other than $S U(2)$, as long as they allow for non-trivial homotopies. This can be 
achieved by simply adjusting the normalisation of the winding number. The case of $S U(N)$ is identical, since one can consider an $S U(2) \cong S^{3}$ subgroup in $S U(N)$, which maps onto the space-time. Let us take the simplest example that differs from $S U(2)$, the case of $S O(3) \cong S U(2) / \mathbb{Z}_{2}$. The normalisation of $w_{S O(3)}(g)$ is half that of $S U(2)$, since $\operatorname{vol}[S O(3)]=\frac{1}{2} \operatorname{vol}[S U(2)]$. If $w_{S O(3)}(g)=n$, then the shift of the action becomes

$$
S_{\mathrm{CS}} \rightarrow S_{\mathrm{CS}}+4 \pi^{2} \kappa n
$$

Setting $\kappa=\frac{k}{4 \pi}$ leads us to a quantisation condition which requires an even Chern-Simons level $k \in 2 \mathbb{Z}$ in the case of an $S O(3)$ gauge group.

Next, we comment on the quantisation of the Chern-Simons level in the case of a compact abelian gauge group. The same argument, where we compactify $\mathbb{R}^{3} \rightarrow S^{3}$ and then perform gauge transformations with a non-trivial winding number, no longer applies for an abelian group, since $\pi_{1}\left(S^{3}\right)$ is trivial. In fact, the level for an abelian CS theory is not in general quantised [109]. We can, however, derive a quantisation condition with a small set of additional assumptions, more specifically these are

○ Finite Temperature $T$.

Studying a QFT at finite temperature is equivalent to performing a Wick rotation into Euclidean space and compactifying the time direction to an $S^{1}$. This leads to a space-time of the form $S^{1} \times M$, where $M$ is a two dimensional manifold. More information on the development of finite temperature field theory can be found in Kadanoff et al. [110].

- Consistency of the theory in the presence of a magnetic monopole.

As we have already touched upon in the Introduction, in 1931, Paul Dirac considered the possibility of the existence of a magnetic monopole [15]. This consideration is contrary to the statement of Gauss's law for magnetism, which states that magnetic monopoles cannot exist (since for such a configuration we would have $\boldsymbol{\nabla} \cdot \boldsymbol{B} \neq 0$ ). Dirac's construction required the existence of a singularity associated with itself. Later on, in 1975, Wu E Yang [111, 112] resolved the mystery of the Dirac string by 


\section{BACKGROUND}

showing how gauge fields are connected to the mathematical concept of fibre bundles, thus showing that a proper definition of the vector potential of a monopole should be defined in patches, which are related by gauge transformations on the intersections. Here we will consider the monopole on a sphere $S^{2}$. The presence of a monopole implies that

$$
\frac{1}{2 \pi} \int_{S^{2}} F_{12}=1 .
$$

With this setup, we can now perform a large gauge transformation along the time manifold $S^{1}$, and study its effect on the Chern-Simons action. However, this would lead us to a result that is incorrect. The reason for this is that our definition of the Chern-Simons action is ambiguous for a non-trivial fibre bundle (read for a gauge field that is not globally defined). The correct way of approaching this problem is by defining the $U(1)$ Chern-Simons action as the boundary term of a 4-dimensional action [44]

$$
S=\frac{k}{4 \pi} \int_{B} F \wedge F,
$$

where $B$ is a four manifold that bounds $S^{1} \times S^{2}$. Here we shall take $B=D \times S^{2}$, where $D$ is the disk with boundary $S^{1}$. We know that the size of the $S^{1}$ is $\beta=\frac{1}{T}$, as dictated by thermodynamics. This implies that the radius of the disk is $R_{D}=\frac{\beta}{2 \pi}$. Since the total space-time is a direct product, we can write the field strength as a sum of contributions to the separate parts of the manifold

$$
F=F_{D}+F_{S^{2}} .
$$

Since the wedge product of 2 -forms commutes, we have

$$
S=\frac{k}{4 \pi} \int_{B} F \wedge F=\frac{k}{2 \pi} \int_{B} F_{D} \wedge F_{S^{2}} .
$$

Let us consider a large gauge transformation on $S^{1}$. Let $A_{D}$ be the gauge field defined on the disk $D$ and let a position on that disk be described in polar coordinates $(r, \alpha)$, where $r$ is the radial position and $\alpha$ the polar angle. A candidate 
for a non-single valued function on the boundary of $D\left(S^{1}\right)$ is a transformation of the form

$$
A_{D} \rightarrow A_{D}+\frac{r}{R_{D}} d \alpha
$$

This is not a gauge transformation on $D$, but it is a perfectly well-defined gauge transformation on $S^{1}$ and, as such, we expect the boundary theory to be invariant under it. Since 1.20 is not a gauge transformation on $D, F_{D}$ changes

$$
F_{D} \rightarrow F_{D}+d(r d \alpha)=F_{D}+d r \wedge d \alpha
$$

This implies that the action changes accordingly

$$
\begin{aligned}
\delta S & =\frac{k}{2 \pi} \int_{B} \frac{1}{R_{D}} d r \wedge d \alpha \wedge F_{S^{2}} \\
& =\frac{k}{2 \pi} \oint_{S^{1}} d \alpha \int_{S^{2}} F_{S^{2}}=2 \pi k
\end{aligned}
$$

where we have used Stokes' theorem, the monopole flux condition 1.16 and the fact that on the $S^{1}, r=R_{D}$. Just as in the non-abelian case, we see that if we would like the path integral over the boundary to remain invariant, we require that

$$
\delta S \in 2 \pi \mathbb{Z} \Longrightarrow k \in \mathbb{Z}
$$

We remark that if the local definition of the Chern-Simons action had been used in this derivation, we would be off by a factor of 2 , which would mean that the level would be quantised to only even integers, leading to incorrect physical predictions. Finally, it is important to note that even though the arguments above are classical in nature, there is a result that guarantees the absence of radiative corrections of the Chern-Simons level beyond 1-loop. This is known as the Coleman-Hill theorem [113, 114]. 


\section{Equations of Motion}

Let us look at some physical properties that the action functional $S_{\mathrm{CS}}$ possesses. Performing the functional variation, we get the equation of motion $F_{\mu \nu}=0$, implying only pure gauge solutions exist. This might make one wonder, what could possibly be so interesting about CS theory if the classical equations of motion are trivial!? The reason that this theory is in fact interesting and non-trivial, despite the fact that $F=0$, is two-fold. Firstly, CS is a topological field theory. It has trivial local properties in the sense that local observables vanish. All of the physics is encoded in the topology of the manifold and in the non-local observables such as Wilson loops that require us to study the full quantum theory. This was done by Witten [115] in 1989, when he showed that the expectation values of Wilson loops can be expressed in terms of their Jones polynomial - a knot invariant that characterises the loop - thus establishing a deep connection between QFT and knot theory.

Secondly, CS teaches us the old lesson that sometimes the whole is greater than the sum of its parts. In this case, adding matter or a Maxwell term to the Lagrangian alters the pure Maxwell/matter theories immensely. Let us see how these alterations play out.

\subsubsection{Chern-Simons-Maxwell theory}

Here we consider the familiar Maxwell theory deformed by a Chern-Simons term

$$
S_{\mathrm{MCS}}=\int d^{3} x\left[\frac{-1}{4 g^{2}} F_{\mu \nu} F^{\mu \nu}+\frac{k}{4 \pi} \epsilon^{\mu \nu \rho} A_{\mu} \partial_{\nu} A_{\rho}\right] .
$$

This leads to the equation of motion

$$
\partial_{\mu} F^{\mu v}+\frac{k}{4 \pi} g^{2} \epsilon^{v \alpha \beta} F_{\alpha \beta}=0
$$

If we rewrite this equation in terms of the dual field strength $\tilde{F}_{\mu}=\frac{1}{2} \epsilon_{\mu \nu \rho} F^{v \rho}$ by contracting with an $\epsilon$ tensor density, followed by a $\partial$ contraction, and then using the equations of motion again, and finally noting that $\partial_{\mu} \tilde{F}^{\mu}=0($ Bianchi 
identity), we arrive at

$$
\left[\partial_{\mu} \partial^{\mu}+\left(\frac{k}{2 \pi} g^{2}\right)^{2}\right] \tilde{F}^{v}=0 .
$$

Observing that $\tilde{F}^{\mu}$ is gauge-invariant, we deduce that this equation describes a propagating wave with mass $\left(\frac{k}{2 \pi} g^{2}\right)$. Even though in this work we shall promptly be ignoring the Maxwell contribution to the planar physics, it is important to note that despite the fact that the gauge bosons are heavy, we still observe global effects. This provides us with a more general lesson about CS. Namely, that we can have significant long-range global effects, such as the anyonic phase change interaction, even in the case of a gapped phase (such as the quantum Hall droplet). Next, we turn to the matter sector.

\subsubsection{Chern-Simons-matter theory}

We begin by coupling the Chern-Simons gauge field to a general conserved matter current

$$
S=\int d^{3} x \frac{k}{4 \pi} \epsilon^{\mu v \rho} \operatorname{Tr}\left[A_{\mu} \partial_{v} A_{\rho}+\frac{2}{3} A_{\mu} A_{v} A_{\rho}\right]+J^{\mu} A_{\mu}
$$

This action leads to a modified equation of motion

$$
\frac{k}{4 \pi} \epsilon^{\mu v \rho} F_{v \rho}^{a}=J^{\mu a}
$$

where $a=1, \ldots, \operatorname{dim}(\mathbb{b})$ and $F_{\mu \nu}^{a}=\partial_{\mu} A_{v}^{a}-\partial_{\nu} A_{\mu}^{a}+\left[A_{\mu}, A_{v}\right]^{a}$. Note that covariant current conservation is equivalent to the non-abelian Bianchi identity

$$
D_{\mu} J^{\mu a}=\frac{k}{4 \pi} \epsilon^{\mu v \rho} D_{\mu} F^{v \rho a}=0,
$$

where $D_{\mu} F_{v \rho}=\partial_{\mu} F_{v \rho}+\left[A_{\mu}, F_{v \rho}\right]$.

Restricting ourselves to the abelian case, we examine the equations of mo- 
tion component by component. If $J_{\mu}=\left(\rho, J^{i}\right)$ then

$$
\begin{aligned}
\rho & =\frac{k}{2 \pi} B, \\
J^{i} & =\frac{k}{2 \pi} \epsilon^{i j} E_{j},
\end{aligned}
$$

where $B$ is the magnetic field perpendicular to the plane and $E_{j}$ is the electric field. The first equation (1.31) relates the charge density to the magnetic field. So for a non-zero value of $k$, we find that anywhere there is charge, there is also magnetic flux penetrating through it perpendicular to the plane. This is something quite unlike Maxwell theory, where charge movement generates a magnetic field, as opposed to charge presence. This is illustrated in Figure (1.2) for a collection of localised charge distributions. The second equation 1.32 ensures that this charge-flux relation is preserved under time evolution. If we act with $\partial$ on 1.32 then we arrive at

$$
\begin{aligned}
\partial_{i} J^{i} & =\frac{k}{2 \pi} \epsilon^{i j} \partial_{i} E_{j} \\
& =\frac{k}{2 \pi} \epsilon^{i j} \partial_{i}\left(\partial_{j} A_{0}-\partial_{0} A_{j}\right) \\
& =-\frac{k}{2 \pi} \dot{B}=-\dot{\rho},
\end{aligned}
$$

which is just the continuity equation $\dot{\rho}+\partial_{i} J^{i}=0$.

The final aspect of Chern-Simons theory that we will use is the fact that it is a topological quantum field theory (TQFT) of Schwarz type. This means that the theory is independent of the metric. This consequently implies that the CS term does not contribute to the energy-momentum tensor, since

$$
T^{\mu v}=\frac{-2}{\sqrt{-g}} \frac{\delta S_{\mathrm{CS}}}{\delta g_{\mu v}}=0 .
$$

One way to see that CS is a topological field theory is by writing it as an integral of a differential form

$$
S_{\mathrm{CS}}=\frac{k}{4 \pi} \int(A \wedge d A+A \wedge A \wedge A)
$$




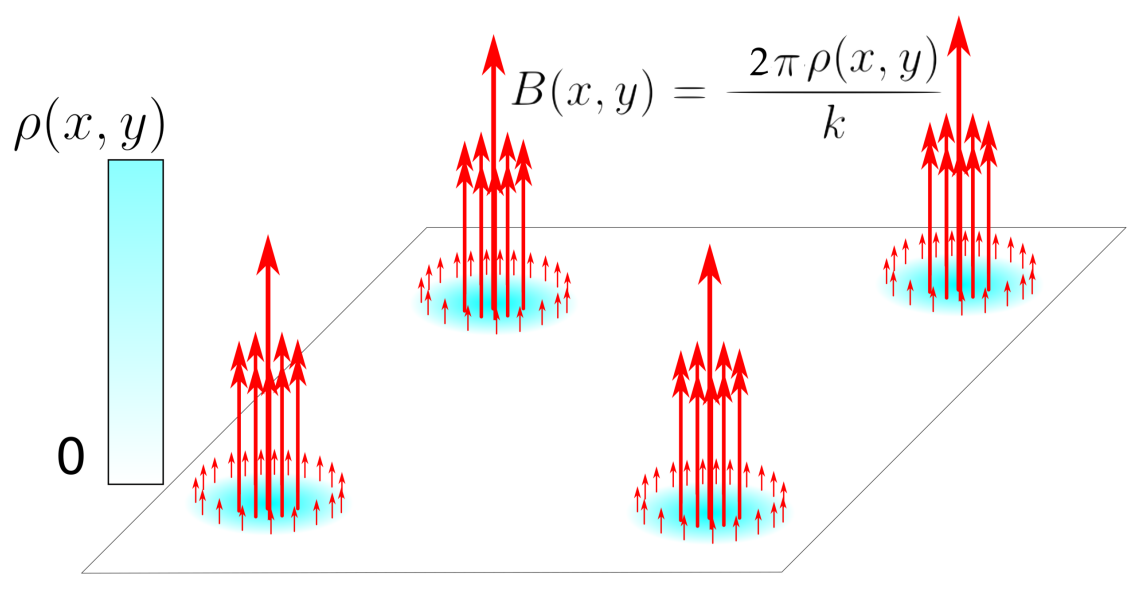

Figure 1.2: A collection of localised charge distributions, with magnetic flux lines of strength $\frac{2 \pi \rho(x, y)}{k}$ tied to the charges. Here the charge density distribution is denoted by $\rho(x, y)$ and the magnetic field strength is denoted by $B(x, y)$. Dark shades of cyan depict a large charge density and large red vectors are associated with a strong magnetic field perpendicular to the plane. The charge and flux are tied together throughout the motion of the particles as a result of the ChernSimons equations 1.31 - 1.32.

This construction does not require the metric to be defined, unlike the YangMills term, which involves the Hodge star (a metric dependent construct) in order to be defined through differential forms.

This concludes our review of CS theory. We now move on to a refresher of statistical physics.

\subsection{Statistical Physics}

Here we motivate the study of QFT as a gateway into understanding phenomena in many body physics. Physical phenomena at macro scale require micro scale explanations. The transition between a microscopic theory and its observable large scale properties is facillitated by the use of techniques from statistical mechanics. Here we review the connection between the microscopic Lagrangian formulation and the statistical partition function through the formalism of the path integral. We follow through by accentuating the treatment 
of a chemical potential and the subtleties related to it in Subsection 1.2.2. We build up to this with standard statistical physics material in Subsection 1.2.1.

\subsubsection{Functional integral representation of the partition func- tion}

In this section we derive the functional integral representation of the partition function for interacting, relativistic, non-gauge field theories. Good reviews on the techniques we discuss here can be found in Feynman E Hibbs's classic work on the path integral formulation [116] and a set of excellent lecture notes on thermal field theory by Laine $\mathcal{E}$ Vuorinen [117]. Let $\hat{\phi}(x, 0)$ be a Schrödinger picture field operator at time $t=0$. We define its conjugate momentum operator to be $\hat{\pi}(x, 0)$. The eigenstates of the field operator are labelled $|\phi\rangle$ and satisfy

$$
\hat{\phi}(x, 0)|\phi\rangle=\phi(x)|\phi\rangle,
$$

where $\phi(x)$ is the eigenvalue of $|\phi\rangle$. This is complemented with the completeness and orthogonality relation

$$
\begin{array}{r}
\int d \phi(x)|\phi\rangle\langle\phi|=1, \\
\left\langle\phi_{a} \mid \phi_{b}\right\rangle=\prod_{x} \delta\left(\phi_{a}(x)-\phi_{b}(\boldsymbol{x})\right) .
\end{array}
$$

And similarly for the conjugate momentum

$$
\begin{array}{r}
\hat{\pi}(\boldsymbol{x}, 0)|\pi\rangle=\pi(\boldsymbol{x})|\pi\rangle, \\
\int d \pi(\boldsymbol{x})|\pi\rangle\langle\pi|=1, \\
\left\langle\pi_{a} \mid \pi_{b}\right\rangle=\prod_{x} \delta\left(\pi_{a}(\boldsymbol{x})-\pi_{b}(\boldsymbol{x})\right) .
\end{array}
$$


Suppose we have a Hamiltonian with no explicit time dependence

$$
H=\int d^{2} x \mathcal{H}(\hat{\phi}, \hat{\pi})
$$

Assume that a system is in a state $\left|\phi_{a}\right\rangle$ at time $t=0$. After a time $t_{f}$ it evolves to $e^{-i H t_{f}}\left|\phi_{a}\right\rangle$, assuming that the Hamiltonian has no explicit time dependence. The transition amplitude for going from state $\left|\phi_{a}\right\rangle$ to state $\left|\phi_{b}\right\rangle$ after a time $t_{f}$ is $\left\langle\phi_{b}\left|e^{-i H t_{f}}\right| \phi_{a}\right\rangle$. In order to study a system at thermodynamic equilibrium, we are interested in the amplitude for the system returning to the same state after a time $t_{f}$. To be able to compute this amplitude, we divide the time interval $\left(0, t_{f}\right)$ into $\ell$ equal time steps $\Delta t=\frac{t_{f}}{\ell}$. Then at each time interval, we insert a complete set of states, alternating between field operators and their conjugate momenta

$$
\begin{aligned}
\left\langle\phi_{a}\left|e^{-i H t_{f}}\right| \phi_{a}\right\rangle & =\lim _{\ell \rightarrow \infty} \int\left(\prod_{i=1}^{\ell} \frac{d \pi_{i} d \phi_{i}}{2 \pi}\right) \\
& \times\left\langle\phi_{a} \mid \pi_{\ell}\right\rangle\left\langle\pi_{\ell}\left|e^{-i H \Delta t}\right| \phi_{\ell}\right\rangle\left\langle\phi_{\ell} \mid \pi_{\ell-1}\right\rangle \\
& \times\left\langle\pi_{\ell-1}\left|e^{-i H \Delta t}\right| \phi_{\ell-1}\right\rangle \ldots \\
& \times\left\langle\phi_{2} \mid \pi_{1}\right\rangle\left\langle\pi_{1}\left|e^{-i H \Delta t}\right| \phi_{1}\right\rangle\left\langle\phi_{1} \mid \phi_{a}\right\rangle .
\end{aligned}
$$

In order to evaluate this expression, we need to do several things. First of all, from single particle quantum mechanics, we know that

$$
\langle x \mid p\rangle=e^{i p \cdot x}
$$

So an appropriate generalisation of this inner product from phase space coordinates to field operators is

$$
\left\langle\phi_{i+1} \mid \pi_{i}\right\rangle=\exp \left(i \int d^{2} x \pi_{i}(x) \phi_{i+1}(\boldsymbol{x})\right) .
$$


Since $\Delta t \rightarrow 0$, we can expand as follows, keeping terms up to first order:

$$
\begin{aligned}
\left\langle\pi_{i}\left|e^{-i H_{i} \Delta t}\right| \phi_{i}\right\rangle & \sim\left\langle\pi_{i}\left|\left(1-i H_{i} \Delta t\right)\right| \phi_{i}\right\rangle \\
& =\left\langle\pi_{i} \mid \phi_{i}\right\rangle\left(1-i H_{i} \Delta t\right) \\
& =\left(1-i H_{i} \Delta t\right) \exp \left(-i \int d^{2} x \pi_{i}(\boldsymbol{x}) \phi_{i}(\boldsymbol{x})\right)
\end{aligned}
$$

where

$$
H_{i}=\int d^{2} x \mathcal{H}\left(\pi_{i}(\boldsymbol{x}) \phi_{i}(\boldsymbol{x})\right)
$$

In the end we arrive at

$$
\begin{aligned}
\left\langle\phi_{a}\left|e^{-i H t_{f}}\right| \phi_{a}\right\rangle & =\lim _{\ell \rightarrow \infty} \int\left(\prod_{i=1}^{\ell} \frac{d \pi_{i} d \phi_{i}}{2 \pi}\right) \delta\left(\phi_{1}-\phi_{a}\right) \\
& \times \exp \left(i \Delta t \sum_{j=1}^{\ell} \int d^{2} x\left[\frac{\pi_{j}\left(\phi_{j+1}-\phi_{j}\right)}{\Delta t}-\mathcal{H}\left(\pi_{j}, \phi_{j}\right)\right]\right) .
\end{aligned}
$$

Taking the continuum limit we arrive at the path integral representation of the partition function

$$
\begin{aligned}
& \left\langle\phi_{a}\left|e^{-i H t_{f}}\right| \phi_{a}\right\rangle=\int \mathcal{D} \pi \int_{\phi(x, 0)=\phi_{a}(x)}^{\phi\left(x, t_{f}\right)=\phi_{a}(x)} \mathcal{D} \phi \\
& \times \exp \left[i \int_{0}^{t_{f}} d t \int d^{2} x\left(\pi(\boldsymbol{x}, t) \frac{\partial \phi(\boldsymbol{x}, t)}{\partial t}-\mathcal{H}(\phi(\boldsymbol{x}, t), \pi(\boldsymbol{x}, t))\right)\right] .
\end{aligned}
$$

For a Hamiltonian that is quadratic in the canonical momenta, we can just complete the square, integrate out the momenta and arrive at the usual expression for a transition amplitude in terms of a path integral over $e^{i S}$. Since our purpose here is to illuminate the connection between the statistical partition function and the path integral, we shall perform a few more manipulations. 


\subsubsection{Grand canonical ensemble}

With the background of the previous section in place, we are now prepared to tackle the study of the grand canonical partition function in the path integral formalism. First, we note that the grand canonical partition function is defined as follows

$$
Z=\operatorname{Tr}\left[e^{-\beta(H-\mu N)}\right]
$$

where $\beta=\frac{1}{k_{B} T}$ is the inverse temperature, $\mu$ is the chemical potential, and $N$ is the particle number. To see that $N$ denotes particle number, we note that in a QFT, $N$ will be associated with a conserved charge. Hence $N$ counts the charge in the system, which, for particles with discrete charge, translates directly to counting the number of particles. We can express the trace in 1.50 in the $\phi$ basis

$$
Z=\int d \phi_{a}\left\langle\phi_{a}\left|e^{-\beta(H-\mu N)}\right| \phi_{a}\right\rangle
$$

We see that this expression is very similar to 1.49 . In order to match the two expressions, we need to do three things. First, we set $t \rightarrow-i \tau$, such that $t_{f} \rightarrow-i \beta$. Then we shift the Hamiltonian density in order to account for the inclusion of a chemical potential

$$
\mathcal{H}(\phi(x, t), \pi(x, t)) \rightarrow \mathcal{H}(\phi(x, t), \pi(x, t))-\mu \mathcal{N}(\phi(x, t), \pi(x, t))
$$

where $\mathcal{N}$ is a number density. Finally, we include the trace operation, which integrates over all possible boundary conditions. In the end we are left with

$$
Z=\int \mathcal{D} \pi \int_{\text {periodic }} \mathcal{D} \phi \exp \left[\int_{0}^{\beta} \int d^{2} x\left(\pi \frac{\partial \phi}{\partial t}-\mathcal{H}(\pi, \phi)+\mu \mathcal{N}(\pi, \phi)\right)\right] .
$$

This is the path integral representation of the partition function of the grand canonical ensemble of a single real scalar field. This expression is readily generalisable to more fields by integrating over the extra fields and their respective conjugate momenta. 
Here we shall make a few observations that will come in use frequently for the rest of this thesis. For the purposes of presentation, we will elaborate on these points in the context of a somewhat simpler model. This should not alarm the reader, because the properties we are discussing are general and model independent. With this in mind, we consider the Lagrangian description of a complex relativistic scalar in $2+1$ dimensions with a potential $U(\phi)$

$$
\mathcal{L}=\left(\partial_{\mu} \phi^{*} \partial^{\mu} \phi-U(|\phi|)\right)
$$

This system possesses a global symmetry

$$
\phi \rightarrow e^{i \alpha} \phi, \quad \phi^{*} \rightarrow e^{-i \alpha} \phi^{*}
$$

This leads to a conserved Noether current and consequently a conserved charge

$$
J_{0}=-i \int d^{2} x\left(\pi \phi-\pi^{\dagger} \phi^{\dagger}\right)
$$

where $\pi=\frac{\delta \mathcal{L}}{\delta\left(\partial_{0} \phi\right)}$ is the canonical momentum. Based on the assumption of identical particles with discrete charges, we postulate that $J_{0}=\mathcal{N}$. Next, we perform the momentum integrals in (1.53). Since now we have a complex field, we have to integrate over all of $\phi, \phi^{\dagger}, \pi$ and $\pi^{\dagger}$

$$
\begin{aligned}
Z & =\int \mathcal{D} \pi \mathcal{D} \pi^{\dagger} \int_{\text {periodic }} \mathcal{D} \phi \mathcal{D} \phi^{\dagger} \\
& \times \exp \left[\int_{0}^{\beta} \int d^{2} x\left(\pi \frac{\partial \phi}{\partial t}+\pi^{\dagger} \frac{\partial \phi^{\dagger}}{\partial t}-\mathcal{H}\left(\pi, \pi^{\dagger}, \phi, \phi^{\dagger}\right)-i \mu\left(\pi \phi-\pi^{\dagger} \phi^{\dagger}\right)\right)\right] \\
& =\int \mathcal{D} \pi \mathcal{D} \pi^{\dagger} \int_{\text {periodic }} \mathcal{D} \phi \mathcal{D} \phi^{\dagger} \\
& \times \exp \left[\int_{0}^{\beta} \int d^{2} x\left(\pi\left(\frac{\partial \phi}{\partial t}-i \mu \phi\right)+\pi^{\dagger}\left(\frac{\partial \phi^{\dagger}}{\partial t}+i \mu \phi^{\dagger}\right)-\mathcal{H}\left(\pi, \pi^{\dagger}, \phi, \phi^{\dagger}\right)\right)\right]
\end{aligned}
$$

Performing the path integral leaves us with

$$
Z=\int_{\text {periodic }} \mathcal{D} \phi \mathcal{D} \phi^{\dagger} \exp \left[\int_{0}^{\tau} d \tau \int d^{2} x \mathcal{L}^{\prime}\right],
$$


where $\mathcal{L}^{\prime}$ is the same as $\mathcal{L}$ except that the time derivatives acting on $\phi$ instead act as covariant derivatives in the presence of a constant background gauge field $A_{0}=\mu$, i.e.

$$
\partial_{0} \rightarrow \partial_{0}-i \mu
$$

This is a general result that we will be making use of numerous times.

\section{Chemical potential for a local symmetry}

Now, let us see how the grand canonical partition function changes when we try to introduce a chemical potential for a gauged symmetry. First, we modify the Lagrangian $\mathcal{L}$ by gauging the $U(1)$ and adding a Maxwell term $-\frac{1}{4 g^{2}} F_{\mu \nu} F^{\mu \nu}$. For simplicity, let us assume that the potential has the monomial super-renormalisable form

$$
U(|\phi|)=\lambda|\phi|^{4}
$$

Therefore the Lagrangian is

$$
\mathcal{L}=\left(D_{\mu} \phi\right)^{*} D^{\mu} \phi-\lambda|\phi|^{4}-\frac{1}{4 g^{2}} F_{\mu \nu} F^{\mu \nu},
$$

where $D_{\mu}=\partial_{\mu}-i A_{\mu}$. According to the rule 1.59 that we established above,

$$
\mathcal{L} \rightarrow \mathcal{L}^{\prime}=\mathcal{L}\left(\phi, D_{\mu}^{\prime} \phi, A_{\mu}\right)
$$

where $D_{\mu}^{\prime}=D_{\mu}-i \mu \delta_{\mu 0}$. Let us also express $\phi$ in terms of a modulus and a phase

$$
\phi(x)=\sigma(x) e^{i \alpha(x)} .
$$


If we assume a homogenous, constant configuration, the equations of motion tell us that

$$
\begin{aligned}
\sigma & =\sqrt{\frac{\mu^{2}}{2 \lambda}}, \\
\frac{1}{g^{2}} \partial_{\mu} F^{\mu 0} & =\mu \sigma^{2} .
\end{aligned}
$$

The first equation tells us that the scalars have condensed to the ground state. The second equation states that we have a non-zero charge density in this ground state. On the one hand, this makes sense, since the charged scalars have condensed. On the other hand, this is contradictory, since we are studying a system at equilibrium and a charged system cannot be in equilibrium by definition. In order to fix this problem, we introduce a background charge density, which guarantees that the system is neutral

$$
\mathcal{L}^{\prime} \rightarrow \mathcal{L}^{\prime}-A_{0} J_{0}
$$

where $J_{0}=\frac{\mu^{3}}{2 \lambda}$. This way of thinking about a chemical potential of a local symmetry was first suggested by Kapusta [118].

The statistical description of weakly interacting particles, for example in a fluid, can be thought of as the same as that of a free gas of non-interacting quasiparticles that are in one to one correspondence with the original interacting excitations of the material [119]. We find that in order to explain some of the surprising features of the charge carriers in a quantum Hall system, we need to go beyond the weakly interacting description of a Landau-Fermi liquid.

\subsection{Fractional Quantum Hall Effect}

Here we discuss what is perhaps the most applied aspect of TQFT in the real world. But before we get to the point of discussing the highly non-trivial physics of the various types of quantum Hall effects, we take a moment to review the classical picture. 


\subsubsection{Classical Hall effect}

In the year 1879, Edwin Hall [49] set up an experiment consisting of a conductor plate with two electrodes attached at opposite ends of the plate and a magnetic field perpendicularly penetrating the surface of the conductor. He found that he could measure a non-zero potential across the conductor, orthogonal to the plane defined by the driving current and the magnetic field. The generation of this potential difference has since been known as the Hall effect. Despite this being an exciting discovery at the time, from our modern perspective, this can all be explained through basic knowledge of conductors and classical electrodynamics. In the presence of a magnetic field, the charge carriers, which are responsible for the current in the conductor, get deflected in a direction orthogonal to their motion due to the Lorentz force

$$
\boldsymbol{F}=q(\boldsymbol{E}+\boldsymbol{v} \times \boldsymbol{B})
$$

where $q$ is the charge of the electrons, $E$ is the electric field due to the potential difference in the electrodes, $\boldsymbol{v}$ is the velocity of the charge carriers and $\boldsymbol{B}$ is the magnetic field in the material. If we account for the possible collisions that may occur within a sample of the conductor, which cause the charge carriers (in this case electrons) to slow down, we arrive at a slightly modified equation to the Lorentz law from above

$$
m \dot{\boldsymbol{v}}=q \boldsymbol{E}+q \boldsymbol{v} \times \boldsymbol{B}-\frac{1}{\tau} m \boldsymbol{v}
$$

where $\tau$ is the scattering time. It accounts for how frequently the electrons scatter, hence a large scattering time makes the last term in 1.68 disappear completely. This description of the charge carriers in a material, subject to both electric and magnetic fields in the presence of impurities, is called the Drude model, named after the German physicist Paul Drude who first proposed it [120, 121]. Before we look at the general solution of this model, we make several remarks about certain limiting cases.

First, assume that there are no collisions (i.e. the collision time diverges, 
$\tau \rightarrow \infty)$ and that there is no electric field. Then the system simplifies to

$$
m \dot{v}=q v \times B
$$

For a particle confined to the plane, the velocity vector is $v=(\dot{x}, \dot{y})$, which leads to the system of two coupled linear ordinary differential equations (ODEs)

$$
m \ddot{x}=q B \dot{y}, \quad m \ddot{y}=-q B \dot{x} .
$$

The general solution to this system is

$$
\begin{aligned}
& x(t)=x_{0}+R \sin \left(\omega_{B} t+\varphi\right), \\
& y(t)=y_{0}+R \cos \left(\omega_{B} t+\varphi\right),
\end{aligned}
$$

where $x_{0}, y_{0}, R, \varphi$ are all integration constants and

$$
\omega_{B}=\frac{q B}{m}
$$

is the cyclotron frequency. We see that the solution in the absence of collisions and an electric potential represents circular motion with a fixed, magnetic field dependent frequency $\omega_{B}$.

Let us go back to the full Drude model. We would like to find out what the equilibrium of the system looks like. This implies that $\dot{v}=0$. The equation of motion becomes

$$
\begin{gathered}
\boldsymbol{v}-\frac{q \tau}{m} \boldsymbol{v} \times \boldsymbol{B}=\frac{\tau q}{m} \boldsymbol{E} \\
{\left[\begin{array}{cc}
1 & -\frac{q \tau B}{m} \\
\frac{q \tau B}{m} & 1
\end{array}\right] \boldsymbol{v}=\frac{\tau q}{m} \boldsymbol{E}}
\end{gathered}
$$

Subsituting $\boldsymbol{J}=q \boldsymbol{v}$ and $\omega_{B}=\frac{q B}{m}$

$$
\left[\begin{array}{cc}
1 & -\omega_{B} \tau \\
\omega_{B} \tau & 1
\end{array}\right] \boldsymbol{J}=\frac{\tau q^{2}}{m} \boldsymbol{E} .
$$


After inverting we arrive at Ohm's law

$$
J=\sigma \boldsymbol{E}
$$

where

$$
\sigma=\frac{q^{2} \tau}{m\left(\tau^{2} \omega_{B}^{2}+1\right)}\left[\begin{array}{cc}
1 & \tau \omega_{B} \\
-\tau \omega_{B} & 1
\end{array}\right]
$$

is the conductivity tensor. It is related to the resistivity tensor by inversion

$$
\rho=\sigma^{-1}=\frac{m}{q^{2} \tau}\left[\begin{array}{cc}
1 & -\tau \omega_{B} \\
\tau \omega_{B} & 1
\end{array}\right]=\left[\begin{array}{ll}
\rho_{x x} & \rho_{x y} \\
\rho_{y x} & \rho_{y y}
\end{array}\right],
$$

where $\rho_{x x}=\rho_{y y}$ and $\rho_{x y}=-\rho_{y x}$.

This equation tells us that in the presence of a constant magnetic field, the charge carriers experience two types of resistivity. One is the usual type of resistivity, that you can expect from a conductor, $\rho_{x x}=\frac{m}{q^{2} \tau}-$ it is independent of the magnetic field and as the scattering time increases, i.e. the number of scattering events decreases, the resistivity decreases. The other type of resistivity is different. The off-diagonal component of the resistivity $\rho_{y x}=\frac{m \omega_{B}}{q^{2}}=\frac{B}{q}$ is independent of $\tau$. So somehow the resistivity in the direction orthogonal to that of the driving current does not depend on the impurities in the material. Another way in which $\rho_{y x}$ is different from $\rho_{x x}$ is that it depends on the magnetic field. Surprisingly, as $B \rightarrow 0$ so does $\rho_{y x} \rightarrow 0$. It would be incorrect to assume that the vanishing of $\rho_{x y}$ implies that we achieve superconductivity, since $\sigma_{x y}$ also vanishes and therefore there is no current being generated perpendicular to the generating electric field.

The Drude model is successful when compared to the classical experimental results obtained by Hall since it predicts correctly that the resistivity $\rho_{x y}$ would grow linearly with the magnetic field $B$. 


\subsubsection{Quantum Hall effect}

Now that we have an understanding of the classical Hall effect, we are ready to delve into the physics of the quantum Hall effect. It is important to distinguish two different types of quantum Hall effects, since they are qualitatively different, occur in different materials and seem to have fundamentally different physical explanations. The first type is the integer quantum Hall effect (IQHE). In 1980, von Klitzing et al. [50] performed the Hall experiment at ultralow temperatures with strong magnetic fields and found that the above relation between $\rho_{y x}$ and $B$ does not hold for large enough magnetic fields. They found that beyond a certain magnetic field strength, plateaux started appearing in the resistivity that could not be explained by the classical picture. Further, they found that in the plateaux, where $\rho_{y x}$ was constant, $\rho_{x x}$ was vanishing. The precise relation that they discovered was

$$
\rho_{y x}=\frac{2 \pi \hbar}{e^{2}} \frac{1}{v}, \quad v \in \mathbb{Z} .
$$

Thus the quantity $\frac{2 \pi \hbar}{e^{2}}$ is dubbed the quantum of resistivity. The explanation of the integer quantum Hall effect is based on the quantum mechanics of noninteracting particles confined to a plane. In the case of the fractional quantum Hall effect (FQHE), where $v \in \mathbb{Q}$, we need to study a highly interacting system, which makes the FQHE a very interesting topic for theoretical physics.

\subsubsection{Landau levels and the integer quantum Hall effect}

Before we attempt to tackle the intricacies of the FQHE, we first acquaint ourselves with the quantum mechanical analogue of the Drude model. Namely, we will be interested in the quantum mechanics of a charged particle confined to a plane, subjected to a magnetic field perpendicular to the plane (for now we will consider the electric field to be vanishing). The physics of this system can be described by a harmonic oscillator. The energy states of this system are today referred to as Landau levels. The name comes from Landau's description of diamagnetism in metals through the quantised orbits of free electrons in metals [51]. Historically, there have been others who have independently 
studied this system [122-124], but Landau's name ended up being attached to it.

For the purpose of quantisation, we consider a system, consisting of a particle in a magnetic field using the Hamiltonian formulation. The general Hamiltonian for a particle at position $x$ in a vector potential $A(x)$ is

$$
H=\frac{1}{2 m}(\boldsymbol{p}+e \boldsymbol{A})^{2}
$$

Here we take the special case of a vector potential such that $\nabla \times A=B \hat{z}$.

In order to solve the quantum system, we perform the canonical quantisation procedure and we promote the positions and momenta to operators

$$
\left[\hat{x}_{i}, \hat{p}_{j}\right]=i \hbar \delta_{i j}, \quad\left[\hat{x}_{i}, \hat{x}_{j}\right]=\left[\hat{p}_{i}, \hat{p}_{j}\right]=0
$$

Here we use Landau gauge

$$
A=x B \hat{y} .
$$

Now we can define raising and lowering operators

$$
\hat{a}^{\dagger}=\hat{p}_{x}+i\left(\hat{p}_{y}+e A_{y}\right), \quad \hat{a}=\hat{p}_{x}-i\left(\hat{p}_{y}+e A_{y}\right) .
$$

Substituting the form of $A$ in Landau gauge and using the canonical quantisation conditions, we get the usual ladder operator commutation relations

$$
\left[\hat{a}, \hat{a}^{\dagger}\right]=1
$$

In terms of $\hat{a}$ and $\hat{a}^{\dagger}$, the Hamiltonian takes the standard form

$$
H=\hbar \omega_{B}\left(\hat{a}^{\dagger} \hat{a}+\frac{1}{2}\right),
$$


where $\omega_{B}=\frac{e B}{m}$. This leads to a tower of energy eigenstates $|n\rangle$ with energies

$$
E_{n}=\hbar \omega_{B}\left(n+\frac{1}{2}\right), \quad n \in \mathbb{N}
$$

Since we are studying the QHE, we expect the magnetic fields to be large, hence the gap between the energy levels would consequently be very large too. If the gap is, in addition, very large in comparison to the temperature of the system, i.e. $k_{B} T \ll \hbar \omega_{B}$, then we expect only the first few Landau levels to be occupied. An important fact to take note of here, is that we are attempting to study a system of a macroscopic number of electrons, yet we see that they occupy only a few energy eigenstates. This tells us that these energy states are extremely degenerate. This degeneracy is what is going to play a role in the conductivity computation.

Let us extend this to include an electric field. We choose the electric field to be in the $x$ direction, which results in an electric potential $\phi=-E x$. With this potential, the Hamiltonian becomes

$$
H=\frac{1}{2 m}(\boldsymbol{p}+e \boldsymbol{A})^{2}-e E x
$$

The current of a particle is given by

$$
\mathbf{I}=-e \dot{\mathbf{x}}=e \frac{(\mathbf{p}+e \mathbf{A})}{m} .
$$

Quantum mechanically, we need to average over the available charge carriers. If we are working in the $k_{b} T \ll \hbar \omega_{B}$ limit, then only the first $v$ Landau levels contribute to the current, so we have

$$
\langle\mathbf{I}\rangle=-\frac{e}{m} \sum_{n=1}^{v} \sum_{k}\langle\psi|-i \hbar \nabla+e \mathbf{A}| \psi\rangle .
$$

The current in the $x$ direction is

$$
I_{x}=-\frac{e}{m} \sum_{n=1}^{v} \sum_{k}\left\langle\psi\left|p_{x}\right| \psi\right\rangle=-\frac{e}{m} \sum_{n=1}^{v} \sum_{k}\left\langle\psi\left|\frac{\hat{a}^{\dagger}+\hat{a}}{2}\right| \psi\right\rangle=0,
$$


where the last term vanishes, since we are summing over eigenstates. The current in the $y$ direction is

$$
I_{y}=e v \sum_{k} \frac{E}{B} .
$$

Assuming that the Landau levels are completely filled, which is the case if the Fermi energy is in the gap between the levels, the sum over $k$ is the number of electrons per Landau level. The number of electrons per Landau level can be approximately derived by taking a unit area $A$ and dividing it by the size of an electron orbit $2 \pi l_{B}^{2}$

$$
\sum_{k} 1=\frac{A B e}{2 \pi \hbar} .
$$

This leaves us with the current density

$$
\mathbf{J}=\left(\begin{array}{c}
0 \\
\frac{-e^{2} v E}{2 \pi \hbar}
\end{array}\right) .
$$

We can again use Ohm's law 1.77) and infer the conductivity tensor

$$
\sigma=\left(\begin{array}{cc}
0 & \frac{-e^{2} v}{2 \pi \hbar} \\
\frac{-e^{2} v}{2 \pi \hbar} & 0
\end{array}\right) .
$$

Inverting this expression and reading the off-diagonal terms, we find the Hall resistivity

$$
\rho_{x y}=-\frac{2 \pi \hbar}{e^{2} v}
$$

The above discussion shows that the IQHE effect comes out of simple quantum mechanics. We have omitted a lot of details and intricacies relating to impurities in the sample, edge states [125], the role of topology, the role of extended states and the irrelevance of localised states [126] and much more. We refer the reader to the following reviews [48, 52, 53] to learn more about these details. The main point that we would like to make here is that the IQHE 


\section{BACKGROUND}

can be understood in terms of a non-interacting system of electrons. This is in contrast to our current understanding of the FQHE, which requires strongly interacting electrons.

\subsubsection{Fractional quantum Hall effect}

Despite the fact that a Nobel prize had already been awarded for the IQHE in 19851, a second QHE Nobel prize ${ }^{2}$ was awarded to Laughlin, Störmer $\mathcal{E}$ Tsui [54, 55] in 1998 for the discovery of the FQHE! This fact alone should signal just how distinct and important the two effects are, despite arising from a system that is classically indisinguishable. As we saw in the IQHE, there is a certain, very large, value of the magnetic field $B$, for which we find the system completely in the lowest Landau level, due to the large energy gap $\Delta E \sim B$. Measuring the resistivity perpendicular to the external electric field, we find it to be equal to

$$
\rho_{x y}=\frac{2 \pi \hbar}{e^{2}}
$$

which corresponds to the case $v=1$. Decreasing the magnetic field, we find resistances corresponding to $v=2,3$,... etc. However, this pattern seems to break down at ultralow temperatures, when the material has high mobility [52]. We start seeing values of the resistance that are no longer in integer values, they are, however, still quantised. More specifically, they are quantised in rational fractional values of the basic unit of resistance $\frac{2 \pi \hbar}{e^{2}}$, hence the name fractional quantum Hall effect. This effect persists going the other direction, in increasing values of the magnetic field, which leads to a fractionally filled lowest Landau level. This is what Störmer, Tsui E Gossard [54] observed in 1982, when they measured the resistance for a fractional filling of $v=\frac{1}{3}$. It appears as though the Landau levels are only fractionally filled, yet the resistance in the direction along the electric field is still vanishing. What could be happening?

\footnotetext{
${ }^{1}$ https://www.nobelprize.org/prizes/physics/1985/summary/

${ }^{2}$ https://www.nobelprize.org/prizes/physics/1998/summary/
} 
It turns out that increasing the electron mobility, or equivalently, reducing the impurities in the material, allows the electrons to more freely interact [127]. This means that our treatment from the previous section, where the electrons were thought of as non-interacting particles, breaks down. In order to understand what is actually happening, we need to solve the interacting electron system. Unfortunately, this is extremely difficult, due to the extremely large number of degrees of freedom. In fact, to this day, there is no known model that can explain all of the fractional values that can be observed in the FQHE. It is an unsolved problem in physics, and as such provides an exciting playground for innovation.

It seems, however, that it is possible to write down a guess that captures the correct qualitative physics and reproduces some of the filling fractions, despite not being the exact solution to the highly interacting system. Such a well-informed guess was first written down not long after the discovery of the fractional states by Laughlin [55] and the distinct features of this guess (such as fractional charge and anyonic statistcs, that we will get to soon) keep informing a lot of the research that is being done both in condensed matter physics and in high energy physics. The wavefunction that he proposed was the following

$$
\psi_{m}(z)=\prod_{j<k}\left(\left(z_{j}-z_{k}\right)^{m}\right) \exp \left(-\frac{1}{4} \sum_{l}\left|z_{l}\right|^{2}\right),
$$

where $z_{j}=x_{j}+i y_{j}$ is the position of the $j^{t h}$ electron and $m$ is an odd natural number $m \in 2 \mathbb{N}+1$. The requirement that $m$ is odd comes from the fact that we are describing electrons, which are fermions, hence we expect the wave function to be completely anti-symmetric under the exchange of any two electrons. Since we have only made continuous changes to the system from the case of the IQHE, we expect the number of states in the lowest Landau level to remain the same. For a unit area $A$, we have $\frac{A}{2 \pi l_{B}^{2}}$ states. If we pick out any given $z_{j}$ from the polynomial prefactor of $\psi_{m}(z)$, we see that it is taken to the power of $(N-1) m$. The exponent of $z_{j}$ is also the eigenvalue of the angular 


\section{BACKGROUND}

momentum operator

$$
J=\hbar\left(z \partial_{z}-\bar{z} \partial_{\bar{z}}\right), \quad J \psi_{m}(z)=\hbar(N-1) m \psi_{m}(z)
$$

This means that we expect a single electron to occupy an area of $A=2 \pi R^{2} \approx$ $2 \pi m(N-1) l_{B}^{2}$. Substituting this into our expression for the number of Landau levels, we have

$$
\text { Degeneracy of lowest Landau levels }=m(N-1) \text {. }
$$

If we take the total number of electrons in the Laughlin wavefunction, and divide it by the number of occupied states, we get the filling fraction

$$
v=\frac{N}{m(N-1)} \stackrel{N \rightarrow \infty}{\longrightarrow} \frac{1}{m} .
$$

We see that the Laughlin wavefunction does indeed provide an explanation for some of the filling fractions.

One can also consider excitations above the Laughlin ground state. These excitations are one of the distinctive elements of the FQH liquid, since they have very peculiar properties. They are quasi-particles in the same sense as phonons, the excitations of an atomic lattice in the Debye model [128]. In the spirit of Landau-Fermi liquid theory [119], one can think of the quasi-particles as renormalised interacting electrons. The first of these peculiar properties is that they have a fractional charge, which is quite surprising, given that the fundamental particles underlying the system have a quantised integer charge. These fractionally charged quasi-particles have been observed experimentally in Hall systems by de Picciotto et al. and Saminadayar et al. [129, 130].

The second surprising feature of these excitations is that they provide an example of an exotic type of particle, which only exists in systems confined to two spatial dimensions. They are particles, whose phase under exchange is neither 1 nor -1 , but somewhere in between. Under an exchange the wavefunction changes as

$$
\psi(x) \rightarrow e^{i \pi \alpha} \psi(x)
$$


where $\alpha \in(0,1)$ is a parameter which characterises the statistics of particles. The value $\alpha=0$ corresponds to bosons, while $\alpha=1$ corresponds to fermions. The quasiparticles in the Laughlin state have a statistics parameter $\alpha=\frac{1}{m}$. In other words, in terms of their statistics, these particles are somewhere between bosons and fermions. So the name given to them is anyons, since they can have any statistics parameter. This property seems to follow from the fractional charge, which we mentioned earlier [131-133]. The existence of particles with statistics which is in between that of fermions and bosons was first speculated by Leinaas \& Myrheim [134] and, later on, by Wilczek [135].

The idea that anyonic statistics exist in $2+1$ dimensions but not in $3+1$ can be understood mathematically through the Braid group [136, 137]. However, going into the details of Braid group representations would take us too far from the main subject of our study. It suffices to say that in $3+1$ spatial dimensions, the interchange of $n$ particles is governed by the symmetric group $S_{n}$, since any world-lines that lead to an exchange can be pulled through and unbraided. This leaves us with either the trivial representation (bosons) of $S_{n}$ or the faithful representation (fermions). On the other hand, in $2+1$ dimensions, this is not the case. In order to exchange two particles, one must move them through space-time. This movement can in general braid the world-lines of particles. This braiding has the effect of leaving a non-trivial exchange phase that is, in general, neither 1 nor -1 .

\subsubsection{Landau-Ginzburg and Chern-Simons theory}

The Laughlin ground state describes a new phase of matter. One which we refer to as the fractional quantum Hall liquid. This phase is stable for small values of $m$ and we know that for very large values of $m$ (very high magnetic fields and small temperature), the two dimensional electron liquid starts to condense into a solid crystal, known as a Wigner crystal [138-140]. The fractional Hall liquid is a very special type of state of matter for several reasons. One of those reasons is that if we attempt to fit it into the accepted LandauGinzburg paradigm in condensed matter physics, we find that we cannot explain some of the observed states [141]. Such attempts to describe the quan- 


\section{BACKGROUND}

tum Hall liquid were first made by Girvin $\mathcal{E}$ McDonald [142], who showed that the Laughlin state contains an off-diagonal-long-range-order in a nonlocal operator. Later on, three separate groups - Zhang, Hansson E Kivelsson, Read and Ezawa E Iwazaki [143-145] showed that the effective field theory describing this state involves Chern-Simons theory. It seems that the fractional quantum Hall liquid belongs to a whole new class of matter, that is not governed by the classification of its symmetries. Rather, it exhibits what is now known as topological order [146, 147]. Topological order is physically characterised by a ground state degeneracy, which depends on the topology of the spatial manifold, quasiparticles with anyonic statistics and non-trivial edge states [146]. All abelian quantum Hall states have been classified in terms of emergent Chern-Simons gauge fields [148].

In 1982, Wilczek observed that a particle current coupled to a CS gauge field produced states with fractional statistics through the binding of particles to flux [132]. We will show how this takes place through the Aharonov-Bohm effect [149, 150]. The existence of charge implies the attachment of flux to charged particles, as we already observed in (1.31). Consider two particles of charge 1 in some units. This means that each of them has a flux $\Phi=\frac{2 \pi}{k}$ attached to them. Suppose we rotate one particle around the other by $180^{\circ}$ and then translate the configuration back to where it was. This action is clearly equivalent to just exchanging the two particles. Whatever this does to the wavefunction, it would have to tell us what type of statistics these particles obey. Performing the aforementioned operation would lead to an AharonovBohm phase in the wave function proportional to

$$
e^{i \frac{1}{2} \oint A \cdot d s}=e^{i \frac{\Phi}{2}}=e^{i \frac{\pi}{k}}
$$

where the initial factor of $\frac{1}{2}$ comes from the fact that we only went half way around the second particle. For $k=0, \infty$ (the 0 case follows from there being no flux in the first place), we have bosons and for $k=1$ we have fermions. So we see that we have found the anyons from Laughlin's ground state for finite values of the CS level $k>1$ !

Yet another feature of the quantum Hall effect pops out of Chern-Simons 
theory when we go back to the equation of motion 1.32 for the electric field in the CS-matter section. We see the off-diagonal conductivity appearing yet again

$$
J^{i}=\sigma_{i j} E^{j}
$$

where $\sigma_{x y}=\frac{k}{2 \pi}$. Inverting this expression and resinstating the units of resistance $\left(\frac{\hbar}{e^{2}}\right)$, we recover the IQHE conductivity for integer $k$

$$
\rho_{x y}=\frac{2 \pi \hbar}{e^{2} k}
$$

But how does CS recover the fractional filling if we have said that $k$ is fixed to be an integer? In order to recover the fractional fillings of the Laughlin state, we postulate the existence of emergent topological degrees of freedom $a_{\mu}$, which we describe through CS theory. Since they emerge out of the collective motions of the interacting electron system, we also expect them to couple to the familiar electromagnetic gauge field $A_{\mu}$. This leads to the effective action

$$
S_{\text {eff }}=\int d^{3} x \frac{k}{4 \pi} \epsilon^{\mu \nu \rho} a_{\mu} \partial_{\nu} a_{\rho}+\frac{1}{2 \pi} \epsilon^{\mu \nu \rho} A_{\mu} \partial_{\nu} a_{\rho}+A_{\mu} J^{\mu}
$$

The equations of motion of this model lead to the relation

$$
\begin{aligned}
J^{\mu} & =\frac{1}{2 \pi} \epsilon^{\mu \nu \rho} \partial_{\nu} a_{\rho}=\frac{1}{4 \pi} \frac{1}{k} \epsilon^{\mu \nu \rho} F_{v \rho} \\
& \Longrightarrow J^{i}=\frac{1}{2 \pi} \frac{1}{k} \epsilon^{i j} E_{j} .
\end{aligned}
$$

Again, inverting this equation and reinstating units, we get the resistivity of the Laughlin states

$$
\rho_{x y}=k \frac{2 \pi \hbar}{e^{2}}, \quad k \in \mathbb{Z} !
$$

One can obtain the resistivity of any abelian Hall system by adding more of these emergent gauge fields, coupling them to each other and computing their effect on the electromagnetic background gauge field. This is done through the 


\section{BACKGROUND}

K-matrix approach [148]. Despite our understanding of the abelian system, there are still fractional fillings that remain unexplained, such as the $v=\frac{5}{2}$ state [151], for example. Finally, we mention that there are other approaches to the understanding of the FQHE, such as Jain's composite fermion [152]. The composite fermion is a bound state of a fermion and an even number of vortices. The fact that such an excitation plays a role in the FQHE, gives us one more reason to be interested in studying vortices, which is the topic that we shall focus on in the next section.

Our exploration of the quantum Hall effect will stop here. We direct the curious reader to a list of very insightful reviews for further exploration of the world of the QHE [48, 52, 53, 103, 146]. What is important to take away from this section is that the understanding of the FQHE is underlied by the study of Chern-Simons theory. Thus studying the models, that are of interest in the present work, might lead to a better understanding of the fascinating problem that is the FQHE. A further connection to this problem is provided by the non-commutative Chern-Simons theory that we explore at the end of this chapter and at the end of Chapter (3). Next, we turn our attention to the study of vortices.

\subsection{Vortices}

This section takes up the task of summarising basic aspects about vortices in general and then more specifically in Chern-Simons-matter theories. Generally, the way we study a QFT is through perturbation theory. We take the free field action and treat all non-linear terms as small perturbations. This leads to predictions about the theory in terms a power series, where the coupling is assumed to be small. Unfortunately, this approach is not sufficient to tell us everything that we need to know about a theory. If one studies the classical equation of motion, one finds that some models possess solutions that diverge in the small coupling limit. Such solutions are referred to as non-perturbative, since they are inaccessible via a perturbative expansion. Specifically, in field theory, we are interested in localised, non-perturbative solutions. Such solutions are called solitons. This name is a kind of portmanteau between a solitary 
wave and the suffix “-on”, generally used for naming particles. The name first appeared in the literature in a paper by Zabusky $\mathcal{E}$ Kruskal [57], studying the Korteweg-de Vries equation - an equation known to exhibit the phenomenon of solitary waves.

Solitons are not just theoretical constructs that come out of our theories. They have found numerous applications in fields such as optics, fluid mechanics, condensed matter physics, quantum field theory and string theory. They are phenomena that are indispensible to our current understanding of strongly coupled field theories. A conjecture of major importance to high energy physics is the idea of a "dual Meissner effect", put forward independently by Nambu, ' $t$ Hooft, Mandelstam for 3+1 dimensions and Polyakov for 2+1 dimensions [3, 153-155]. Their idea is that non-abelian flux tubes, similar to their abelian counterparts that appear in superconductors, form between two quarks. The energy of the non-perturbative flux tube is proportional to its length, which leads to a confining potential for the quarks. This idea plays a central role in the understanding of confinement, and has found a concrete realisation in some supersymmetric models [23, 156], which hints at the possibility that the explanation of confinement in QCD is likely to be based on similar principles.

Another important tool for our understanding of physics at strong coupling are dualities. We shall get to discuss dualities in the next section. For now it suffices to say that duality can sometimes allow us to study a strongly coupled theory through a weakly coupled dual theory. As we hinted at in the Introduction, non-perturbative phenomena play a crucial role in the subject of dualities in any number of dimensions. Since our work is focused on $2+1 \mathrm{di}$ mensional systems, we are most interested in topological solitons, which exist in this number of dimensions. For more details about non-perturbative phenomena in QFT and their applications, the reader may consult Shifman's book on advanced QFT [58].

Vortices are localised, topologically stable, exact solutions to the classical equations of motion in 2 dimensions, which interpolate between two distinct vacua. They may or may not be finite energy configurations. In 1957, Abrikosov showed that if the surface tension between two phases of matter (in 


\section{BACKGROUND}

his case those were superconducting and non-superconducting phases) is negative, then a phase transition would be accompanied by the creation of a lattice of regions, whose interiors reside in a phase different from their exteriors [59]. He also recognised the similarity that these regions of non-superconductivity have with the vortices formed in superfluid helium. This similarity is due to the winding of the phase of the order parameter wave function.

Later on, in 1973, Nielsen E Olesen [60] showed that a solution similar to that of Abrikosov can come about through the study of a microscopic Lagrangian, as opposed to an effective Landau-Ginzburg description. This is the Lagrangian of the abelian Higgs model with a quartic interaction. Similar solutions have been found in the abelian Higgs model with a Chern-Simons interaction by Paul-Khare [61] and in the absence of a Maxwell term by HongKim-Pac [62], Jackiw \& Weinberg [63] and Jackiw, Lee \& Weinberg [64].

\subsubsection{Global $\mathrm{U}(1)$ vortex}

Now we look at the properties of these solutions more closely. Before we look at the gauge theory scenario, we focus on the simplest case of a complex massive scalar with a fourth order interaction, negative mass squared and a global $U(1)$ symmetry. This theory is described by the continuum version of the $X Y$ model, which leads to the Berezinskii-Kosterlitz-Thouless phase transition [6567], which we hinted at in the Introduction. The action functional for this model is

$$
S_{\mathrm{XY}}=\left|\partial_{\mu} \phi\right|^{2}+m^{2}|\phi|^{2}-\lambda|\phi|^{4},
$$

where $m$ is the mass of the scalar and $\lambda$ is the interaction coupling constant. This action leads to the equations of motion

$$
\partial_{\mu} \partial^{\mu} \phi=m^{2} \phi-2 \lambda|\phi|^{2} \phi .
$$

This system has an $S^{1}$ vacuum manifold, parametrised by $\phi=v e^{i \theta}$, where $\theta \in[0,2 \pi)$ is the planar polar angular coordinate and $v^{2}=\frac{m^{2}}{2 \lambda}$. Even before having solved the equations, we can already see the non-perturbative nature 
of the vortex, because a solution would have a size $v \sim \frac{1}{\sqrt{\lambda}}$, which is large for small coupling $\lambda$.

We may consider a field configuration that is $\phi=0$ at the origin but approaches a different point on the vacuum manifold at spatial infinity, where the point depends on $\theta$. More precisely, we are looking at a field $\phi$ such that

$$
\phi \stackrel{r \rightarrow \infty}{\longrightarrow} v e^{i n \theta},
$$

where $n \in \mathbb{Z}$ in order to guarantee that the physical configuration is singlevalued. We would like to compute the energy of such a static circularly symmetric configuration

$$
E=2 \pi \int_{0}^{\infty} r d r\left[\lambda|\phi|^{4}-m^{2}|\phi|^{2}+\left|\phi^{\prime}\right|^{2}+\frac{n^{2} v^{2}}{r^{2}}\right] .
$$

Close to infinity, the potential term vanishes and so does $\phi^{\prime}$. In the end we are left with

$$
E=2 \pi n^{2} v^{2} \int \frac{d r}{r}=2 \pi n^{2} v^{2} \log R
$$

where $R$ is the sample size on which our theory is defined. We see that the global vortex, if it exists, would only have a finite energy for finite samples and is not well-defined in the most general case. It turns out that once the global symmetry is gauged, the divergence disappears and we have a vortex configuration that is well-defined in the infinite volume limit.

\subsubsection{Local $\mathrm{U}(1)$ vortex}

The gauged $U(1)$ vortex, also known as the Abrikosov-Nielsen-Olesen vortex, is again a configuration that interpolates between two different vacua in a theory, but this time we introduce a gauge field, whose dynamics is governed by a Maxwell term and is minimally coupled to a $U(1)$ scalar with a symmetry 
breaking potential. This is the familiar abelian Higgs model

$$
S_{\mathrm{AH}}=\int d^{3} x\left[-\frac{1}{4} F_{\mu v} F^{\mu v}+\left|D_{\mu} \phi\right|^{2}-U(\phi)\right]
$$

where

$$
U(\phi)=\frac{\lambda}{2}|\phi|^{4}-m^{2}|\phi|^{2}
$$

Again, we have a non-trivial manifold of ground states and we can repeat the construction from above. This time the energy of the static radially symmetric configuration ends up slightly different

$$
E=2 \pi \int_{0}^{\infty} r d r\left(\frac{1}{2}\left(B^{2}+E^{2}\right)+\left|D_{\mu} \phi\right|^{2}+U(\phi)\right) .
$$

This time we are aiming to eliminate the divergence that arose from the kinetic term $\left|\partial_{\mu} \phi\right|$. We do this by requiring that $A_{\mu}$ asymptotes to a value that cancels said divergence, in such a way that this value can be arrived through a gauge transformation. This ensures that at $r \rightarrow \infty, A_{\mu}$ is pure gauge, thus yielding a finite contribution to the energy from the electromagnetic field. Such a choice for $A_{\mu}$ is

$$
A_{\mu}=n \partial_{\mu} \alpha(x)
$$

where $\alpha(x)=\theta$. Hence

$$
A_{0}=0, \quad A_{r}=0, \quad A_{\theta}=n .
$$

This implies that

$$
\int_{0}^{\infty} r d r\left|D_{\mu} \phi\right|^{2}=v^{2} \int_{0}^{\infty} r d r \frac{1}{r^{2}}\left|n-A_{\theta}\right|^{2}=0 .
$$

From here we see that the inclusion of a gauge field has cured the divergence and we are left with a sensible infinite volume limit.

Now that we know that these boundary conditions do not lead to a diver- 
gence in the energy, let us see whether a solution with such boundary conditions exists. We write down the equations of motion

$$
\begin{array}{r}
\frac{1}{g^{2}} \partial_{\rho}\left(r F^{\mu \rho}\right)=i r\left[\phi^{*}\left(D^{\mu} \phi\right)-\left(D^{\mu} \phi\right)^{*} \phi\right], \\
\partial_{\mu}\left(r D^{\mu} \phi\right)-i A_{\mu} D^{\mu} \phi+\frac{\delta U}{\delta \phi^{*}}=0 .
\end{array}
$$

After plugging in the ansatz

$$
\phi=\sqrt{\frac{m^{2}}{\lambda}} v(r) e^{i n \theta}, \quad A_{\mu}=n a(r) \delta_{\theta \mu},
$$

and rescaling $r \rightarrow \frac{\hat{r}}{m}$, we arrive at a system of equations for the dimensionless functions $v(r)$ and $a(r)$

$$
\begin{aligned}
\frac{1}{\hat{r}} \frac{d}{d \hat{r}}\left(\hat{r} \frac{d v}{d \hat{r}}\right)-\frac{n^{2}(a-1)^{2} v}{\hat{r}^{2}}-v^{3}+v & =0, \\
\frac{d}{d \hat{r}}\left(\frac{1}{\hat{r}} \frac{d}{d \hat{r}} a\right)-\frac{2 g^{2}}{\lambda \hat{r}}(a-1) v^{2} & =0 .
\end{aligned}
$$

In these variables, the boundary conditions become

$$
\begin{array}{cc}
v(0)=0, & a(0)=0, \\
v(\infty)=1, & a(\infty)=1 .
\end{array}
$$

Proving the existence of a solution to non-linear boundary value problems is, in general, not easy to do. One can show that this problem indeed has a solution for all $n \in \mathbb{Z}$. This was shown for the self-dual case (we explain what this means later in this section) by Taubes [157, 158] and in the general case by Plohr and Berger \& Chen [159, 160]. In general, non-linear boundary value problems are difficult to solve numerically. A common approach generally used is the so-called shooting method. This method takes advantages of the relative simplicity of solving initial value problems to solve the ODE as a function of the initial values. It then uses some form of algebraic solver (e.g. the NewtonRaphson method) in order to find the value of the initial condition, which most 
closely reproduces the correct value for the boundary value problem. Unfortunately, this method does not work well for a wide range of parameters, and numerical solutions become too cumbersome. Instead, here, and in the next chapter, we have employed a collocation method, which uses piece-wise continous cubic polynomials. This method is implemented in MATLAB as the function bvp4c [161]. One can think of this method as a type of multiple shooting method, where a mesh is predefined and a shooting method is applied on the individual intervals.

For small $r$, the solutions to the equations (1.123) and (1.124) look like

$$
\begin{aligned}
& v(r)=J_{n}(r) \sim \frac{r^{n}}{2^{n} n !}, \\
& a(r)=C r^{2} .
\end{aligned}
$$

And asymptotically for large $r$ we have

$$
\begin{aligned}
& v(r)=K_{0}(\sqrt{2} r) \sim 2^{-\frac{3}{4}} \sqrt{\frac{\pi}{r}} e^{-\sqrt{2} r}, \\
& a(r)=r K_{1}\left(\frac{\sqrt{2} g}{\sqrt{\lambda}} r\right) \sim 2^{-\frac{3}{4}} \sqrt{\frac{\pi r \sqrt{\lambda}}{g}} e^{-\sqrt{2} \frac{g}{\sqrt{\lambda}} r} .
\end{aligned}
$$

The system is solved numerically and plotted in Figure 1.3 below. From the asymptotic equations 1.129 and 1.130 , we can recover the masses of the Higgs field and gauge field $m_{H}$ and $m_{a}$, respectively, in the Higgsed phase of the theory

$$
m_{H}=\sqrt{2} m, \quad m_{a}=\sqrt{2} \frac{g^{2}}{\lambda} m
$$

From the form of the propagator for scalar and vector theories, we know that scalar fields act attractively, whereas gauge fields act repulsively for like-charged configurations. This tells us that when $m_{a}>m_{H}$, the scalar field decays slower than the gauge field, so if we were to place two vortices on the plane, we will observe an attractive force between them. Likewise, if $m_{a}<m_{H}$, we expect the vortices to repel each other. This line of thought hints at us that there 

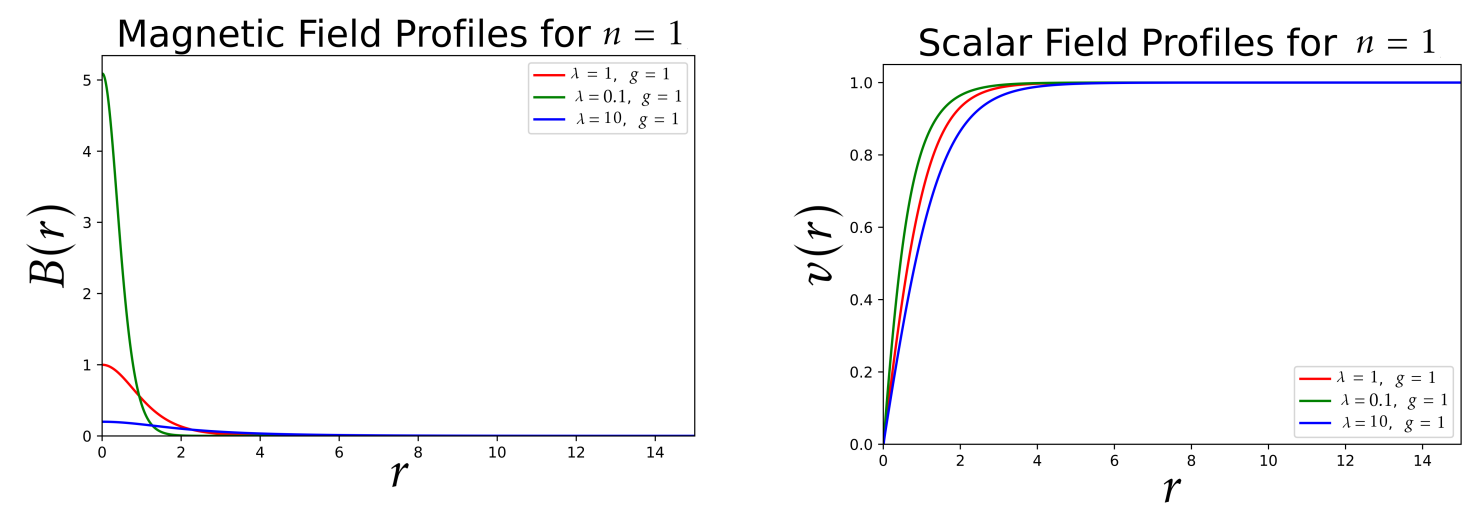

Figure 1.3: The profiles for the magnetic (left) and scalar (right) fields $(B(r)$ and $v(r)$, respectively) are depicted for winding number $n=1$, electromagnetic coupling $g=1$ and quartic interaction coupling $\lambda=(10,1,0.1) \times \lambda_{c}$. Here, $\lambda_{c}$ is the critical coupling, for which the BPS equations (1.137) hold.

is a critical value of the couplings, where vortices are free (non-interacting).

Here we present a more precise argument for this. In order to do this, we use

Bogomol'nyi's identity

$$
\left|D_{1} \phi\right|^{2}+\left|D_{2} \phi\right|^{2}=\left|D_{1} \phi \pm D_{2} \phi\right|^{2} \pm B|\phi|^{2}
$$

Further, we re-express the potential by completing the square

$$
U(\phi)=\frac{\lambda}{2}\left(|\phi|^{2}-v^{2}\right)^{2}-\frac{m^{2} v^{2}}{2} .
$$

Here we can ignore the constant term $\frac{m^{2} v^{2}}{2}$ since the energy is defined up to a constant (albeit an infinite one). Utilising 1.132 and 1.133 we reach the 
following expression for the energy of this configuration

$$
\begin{aligned}
E & =2 \pi \int r d r\left(\frac{1}{2 g^{2}} B^{2} \pm B\left(|\phi|^{2}-v^{2}\right)+\frac{\lambda}{2}\left(|\phi|^{2}-v^{2}\right)^{2}\right. \\
& \left.+\left|D_{1} \phi \pm i D_{2} \phi\right|^{2} \pm B v^{2}\right) \\
& =2 \pi \int r d r\left[\left|D_{1} \phi \pm i D_{2} \phi\right|^{2}+\frac{1}{2}\left(\frac{B}{g} \pm \lambda\left(|\phi|^{2}-v^{2}\right)\right)^{2}\right. \\
& \left. \pm\left(1-\frac{\sqrt{\lambda}}{g}\right) B\left(|\phi|^{2}-v^{2}\right)\right] \pm 2 \pi n v^{2} .
\end{aligned}
$$

The step between (1.134) and 1.135 uses Stokes' theorem

$$
2 \pi \int r d r B=\iint_{\mathbb{R}^{2}}(\nabla \times A) \cdot d S=\oint_{C} A \cdot d \theta=2 \pi n,
$$

where $C$ is the circle at infinity and $A_{\theta} \rightarrow n$ as $r \rightarrow \infty$. In order to have no interaction between the vortices, we need the energy of the $n$ vortex, the bound state of $n$ vortices, to be the same as the energy of $n$ separate vortices. This implies that there is no potential energy, hence no force between them. This condition is satisfied when

$$
\begin{gathered}
\left|D_{1} \phi \pm i D_{2} \phi\right|^{2}=0, \quad \frac{B}{g} \pm \lambda\left(|\phi|^{2}-v^{2}\right)=0, \\
\lambda=g^{2} .
\end{gathered}
$$

The above equations $(1.137)$ and $(1.138)$ ) are known as the BPS equations of the vortex, named after the people who first discovered a non-interacting limit for solitonic solutions in field theory - Bogomol'nyi, Prasad E Sommerfield [68] 69.

\subsubsection{Particle-vortex duality}

The considerations of this section up to now are more general and they apply to more than just systems in $2+1$ dimensions, even though the details differ. In a general QFT, we have roughly two different types of quantum exci- 
tations. One is the familiar type of elementary excitations that appear through the standard procedure of canonical quantisation, where we introduce creation and annihilation operators that insert particles at given positions. The other type is, as we have just seen from the previous section, of a solitonic nature (such as vortices). In other words, a solution that we reach not through expanding around the free field solution in a small coupling parameter, but by solving the classical equations of motion associated with a stationary point parametrically far from the free theory. The size and energy of these solutions is inversely proportional to the coupling, so studying them in the quantum regime becomes tricky. Luckily, because their masses become large at small coupling, they decouple from the theory so one can study the quantum theory of the elementary excitations on its own without worrying about the solitons. Unfortunately, going in the other direction in terms of coupling strength, the solitons become light and their importance grows but so do quantum corrections for the elementary excitations and we reach a regime, where we do not understand the theory. So how do we learn more about these strongly coupled theories?

One approach that has become ever more popular in the past few decades is the idea of a duality. Duality suggests that instead of having one model or one Lagrangian that describes the physics of a system, we instead have two dual theories. For example, a way in which duality can manifest itself is the following:

Theory $I$ has elementary excitations that we can study at weak coupling and non-perturbative excitations which are inaccessible due their large masses. On the other hand, theory II describes the solitons of theory I as its own elementary excitations at weak coupling (and a small mass) and the solitons present in theory $I I$ are in fact the elementary states of theory $I$. If the non-perturbative states in these systems are vortices in $2+1$ dimensions, then we have a type of strong-weak duality called a Particle-Vortex (PV) duality.

PV duality was first studied by Peskin [70] in 1977 and, a few years later, by Dasgupta $\mathcal{E}$ Halperin [71]. The supersymmetric version of the duality is known as mirror symmetry in three dimensions [72]. A non-abelian version of particlevortex duality has been proposed by Murugan $\mathcal{E}$ Nastase [73], which resembles 
the procedure of T-dualising in string theory (introducing a Lagrange multiplier and integrating out the original fields).

Since we now have a reasonable grasp of what vortices are, we are ready to introduce particle-vortex duality. And what better place to start than the abelian Higgs model from the last section (1.115). Earlier, we saw that nonperturbative solutions exist in this theory. It turns out that a model exists where the vortices of the abelian Higgs model appear as elementary excitations. In other words, there is a duality between these two models

$$
S_{\mathrm{AH}} \longleftrightarrow S_{\mathrm{XY}}
$$

where

$$
S_{\mathrm{XY}}=\int d^{3} x\left|\partial_{\mu} \tilde{\phi}\right|^{2}+\tilde{m}^{2}|\tilde{\phi}|^{2}-\frac{\tilde{\lambda}}{2}|\tilde{\phi}|^{4} .
$$

$S_{\mathrm{XY}}$ is the action for the so called $X Y$ model and $S_{\mathrm{AH}}$ is defined as in Equation 1.115. The matching of the physics in the different phases is summarised in Table 1.1. The two models both flow to the same critical point, known as the Wilson-Fisher fixed point [162]. When the parameters are tuned to specific values, we arrive at an interacting CFT. This fixed point coincides with the Gaussian fixed point in $3+1$ dimensions. This fact is one of the aspects that make studying scalars in $2+1$ dimensions interesting.

Moving away from this fixed point, we can consider a non-zero positive mass for the $X Y$ model scalar field. This corresponds to an unbroken global symmetry phase or a Coloumb phase. The excitations in this phase are all massive and the scalars repel each other with a logarithmic potential. This physics is matched on the other side of the duality by a gauge symmetry broken phase. The scalars acquire a vacuum expectation value (VEV), which makes the photon massive, so neither side has a massless excitation. Furthermore, the existence of a VEV allows for the formation of local vortices. These vortices correspond to the elementary excitations $\tilde{\phi}$.

Now we can change the parameters in the opposite direction away from the fixed point, this time with a negative mass squared for the $X Y$ model. This 


\begin{tabular}{|c|c|c|c|}
\hline$\tilde{m}^{2}$ & $>0$ & $=0$ & $<0$ \\
\hline$S_{\mathrm{AH}}$ & $\begin{array}{l}\text { SB phase } \\
\text { local ANO } \\
\text { vortices }\end{array}$ & $\begin{array}{l}\text { Wilson-Fisher } \\
\text { fixed point }\end{array}$ & $\begin{array}{c}\text { Coulomb phase } \\
2 d \text { logarithmic } \\
\text { potential }\end{array}$ \\
\hline$S_{\mathrm{XY}}$ & Massive excitations & $\begin{array}{l}\text { Wilson-Fisher } \\
\text { fixed point }\end{array}$ & $\begin{array}{c}\text { SB phase } \\
\text { global vortices } \\
E \stackrel{V \rightarrow \infty}{\longrightarrow} \infty\end{array}$ \\
\hline
\end{tabular}

Table 1.1: This table shows the matching of the phases of the XY model and the abelian Higgs (AH) model. The $\tilde{m}$ parameter corresponds to the mass in the XY model. Deforming the XY model with a positive mass squared is matched by a negative mass squared deformation on the side of the abelian Higgs model. At $\tilde{m}^{2}=0$, both models are at a critical point known as the Wilson Fisher fixed point. Here, SB stands for "symmetry broken", since the potential breaks the global (local) symmetry of the XY (AH) model, when $\tilde{m}^{2}>0\left(\tilde{m}^{2}<0\right)$.

leads to a symmetry breaking of the global $U(1)$ symmetry. Goldstone's theorem tells us that there is a massless mode. Further, the non-zero VEV implies the existence of global vortices. This physics is matched in the abelian Higgs model by the massless photon, which corresponds to the Goldstone boson of the symmetry broken $X Y$ model. The elementary excitations of the abelian Higgs model attract/repel each other with a logarithmic potential, which is characteristic of two dimensional systems. The global vortices, which also experience such a logarithmic potential, correspond to the elementary excitations of the abelian Higgs model.

No first principle proof of this duality has been shown to this day. In 2016 Karch $\&$ Tong [74] showed that it can be derived if one assumes bosonisation duality as somehow more fundamental. In 1999, Strassler E Kapustin [75] showed that the supersymmetric version of this duality, which we referred to earlier, can be derived via a "Fourier transform-like" operation on the path integral. Despite there being no rigorous first principles proof of the nonsupersymmetric duality, we present here a similar type of transformation that arrives at the correct result, if combined with some physical intuition. This argument is due to Dolan $\mathcal{E}$ Burgess [76] and was further extended by Murugan et al. [77]. 
Our starting point would be the partition function for the abelian Higgs model

$$
\begin{aligned}
Z & =\int \mathcal{D} a_{\mu} \mathcal{D} \Phi_{0} \mathcal{D} \theta \exp \left[\frac{i}{2} \int d^{3} x\left|\left(\partial_{\mu}-i a_{\mu}\right) \Phi\right|^{2}\right] \\
& =\int \mathcal{D} a_{\mu} \mathcal{D} \Phi_{0} \mathcal{D} \theta \exp \left[\frac{i}{2} \int d^{3} x\left(\left|\partial_{\mu} \Phi_{0}\right|^{2}+\left(\partial_{\mu} \theta+a_{\mu}\right)^{2} \Phi_{0}^{2}\right)\right],
\end{aligned}
$$

where we have decomposed the complex scalar $\Phi$ into a magnitude and a phase, $\Phi=\Phi_{0} e^{i \theta}$. Further, we can split up $\theta$ in terms of a smooth and vortex part $\theta=\theta_{\text {smooth }}+\theta_{\text {vortex }}$. The reason for this is that the field $\theta$ can have variations that are both homotopic and not homotopic to the identity. We define $\lambda_{\text {smooth } / \text { vortex }}^{\mu}=\partial^{\mu} \theta_{\text {smooth } / \text { vortex }}$ and we change variables from $\theta$ to $\lambda_{\mu}$. In order for this operation to be self-consistent, we need to impose the constraint $\epsilon_{\rho v \mu} \partial^{v} \lambda_{\text {smooth }}^{\mu}=\epsilon_{\rho v \mu} \partial^{v} \partial^{\mu} \theta_{\text {smooth }}=0$, since $\theta$ is a smooth function. We do this by introducing a Lagrange multiplier field $b_{\mu}$, which leads to the form of the partition function

$$
\begin{gathered}
Z=\int \mathcal{D} a_{\mu} \mathcal{D} \Phi_{0} \mathcal{D} \lambda_{\mu} \mathcal{D} b_{\mu} \exp \left[i \int d ^ { 3 } x \left(\frac{1}{2}\left|\partial_{\mu} \Phi_{0}\right|^{2}+\frac{1}{2}\left(\lambda_{\mu, \text { smooth }}+\lambda_{\mu, \text { vortex }}+a_{\mu}\right)^{2} \Phi_{0}^{2}\right.\right. \\
\left.\left.+\epsilon_{\mu \nu \rho} b^{\mu} \partial^{v} \lambda_{\text {smooth }}^{\rho}\right)\right]
\end{gathered}
$$

Integrating over $b_{\mu}$ returns the original partition function. In order to proceed, we perform the integral over $\lambda_{\mu}$ first. Since the action is quadratic in $\lambda$, integrating $\lambda$ out is equivalent to substituting its equation of motion

$$
\left(\lambda_{\text {smooth }}^{\mu}+\lambda_{\text {vortex }}^{\mu}+a^{\mu}\right) \Phi_{0}^{2}=-\epsilon^{\mu v \rho} \partial_{\nu} b_{\rho}
$$

Alternatively, one can complete the square, perform a Gaussian and arrive at the following form for the partition function

$$
\begin{aligned}
Z=\int \mathcal{D} a_{\mu} \mathcal{D} \Phi_{0} \mathcal{D} \lambda_{\mu} \mathcal{D} b_{\mu} \exp [i & d^{3} x\left(\frac{1}{2}\left(\partial_{\mu} \Phi_{0}\right)^{2}-\frac{1}{4 \Phi_{0}^{2}} f_{\mu \nu}^{b} f^{b \mu \nu}\right. \\
& \left.\left.-\epsilon^{\mu \nu \rho} b_{\mu} \partial_{\nu} a_{\rho}-\epsilon^{\mu \nu \rho} b_{\mu} \partial_{\nu} \partial_{\rho} \theta_{\text {vortex }}\right)\right],
\end{aligned}
$$


where $f_{\mu \nu}^{b}=\partial_{\mu} b_{v}-\partial_{\nu} b_{\mu}$. The last term in the partition function above looks like it should vanish, if $\theta_{\text {vortex }}$ is a smooth function. One can show that this is not the case here using the non-single valuedness of $\theta_{\text {vortex }}$

$$
\begin{aligned}
\iint \epsilon^{0 v \rho} \partial_{\nu} \partial_{\rho} \theta_{\text {vortex }} d^{2} x & =\iint_{S}\left(\nabla \times\left(\nabla \theta_{\text {vortex }}\right)\right) \cdot d S \\
& =\oint_{\partial S} \nabla \theta_{\text {vortex }} \cdot d r \\
& =2 \pi \sum_{a} N_{a, \text { vortex }}
\end{aligned}
$$

where $N_{\text {vortex }}$ is the winding number of the field $\theta$, which signifies the presence of vortices in the system. This implies further that

$$
\epsilon^{0 v \rho} \partial_{\nu} \partial_{\rho} \theta_{\text {vortex }}=\sum_{a} N_{a, \text { vortex }} \dot{y}_{a}(t) \delta\left(x-y_{a}(t)\right)
$$

This shows us that $\epsilon^{0 v \rho} \partial_{\nu} \partial_{\rho} \theta_{\text {vortex }}$ is the 0 th component of a topological current. Hence we define

$$
j_{\text {vortex }}^{\mu}=\epsilon^{\mu \nu \rho} \partial_{\nu} \partial_{\rho} \theta_{\text {vortex }} .
$$

With the above path integral transformation we have exchanged the variable $\theta_{\text {smooth}}$, which is an elementary excitation, with the field $b_{\mu}$, which we have shown to couple with a topological vortex current. This form of the duality is indeed different from the one stated in Equation (1.139), since the dual field is a vector boson. That should not alarm us though, because gauge bosons in $2+1$ dimensions have the same number of degrees of freedom as scalar fields. More precisely, a gauge field in $2+1$ dimensions is equivalent to a compact scalar, which, incidentally, is precisely what the spins in the XY model describe!

We now move on to a more recent development in the study of dualities in 2+1 dimensional theores - Fermi-Bose duality. 


\subsection{Fermi-Bose Duality}

We already met the idea of duality in the previous section. In general, they tend to be an equivalence of two theories, two different ways in which we can state the same physics. Some of them are functional integral analogues of the Fourier transform (for functionals) - a phenomenon can be described either in coordinate or momentum space, by one action or another. This can be achieved by introducing a Lagrange multiplier field and integrating out the original fields [75, 163-165]. Other dualities tend to arise in consequence of the idea of universality. Two models that flow to the same CFT in the infrared (IR) should be equivalent sufficiently close to the fixed point [21, 23]. This is the case with the PV duality we encountered in the previous section. Yet other types of dualities are inspired by the study of black hole thermodynamics and the holographic principle, such as the AdS/CFT duality [35]. Still others seem to be a consequence of deep mathematical ideas, such as the level-rank duality [11, 13, 14, 166, 167].

Whatever the fundamental reasons for their existence are, dualities are interesting because they give us another point of view that allows us to explore and understand the world of fundamental physics in more depth. As Feynman once said, "Every theoretical physicist who is any good knows six or seven different theoretical representations for exactly the same physics" [168]. And in the spirit of his words, here duality is one more representation we wish to understand.

As pointed out above, dualities come in many flavours and the models we are considering here play a role in more than one type. $O(N) / U(N)$ (scalar or fermion) models are holographically dual to higher spin gravitational theories [38]. This duality seems to persist even after deforming the theories with a Chern-Simons term in the large $N$ limit [105]. Fermions in $2+1$ dimensions can be shown to be dual to vector fields via a Fourier transform-like functional integral transformation that we described above [163, 164]. And pure ChernSimons theories are level-rank dual to each other [11, 13, 14, 166, 167]. In this section, we will concentrate on a duality that has all of the above ingredients in some form $(S U(N) / U(N)$ scalars/fermions and CS vector fields) that combine 
into what is believed to be an IR duality. More precisely, we are talking about a set of dualities between $2+1$ dimensional gauge theories that are collectively known as Fermi-Bose duality.

The duality between fermions and bosons was first conjectured in the supersymmetric context [26, 78, 80]. Evidence for the non-supersymmetric version of these dualities comes from large $N$ computations on both sides, both at finite temperature [81] and at $T=0$ [39]. Furthermore, there is compelling evidence that the duality holds at finite $N$ from supersymmetric theories flowing to their non-SUSY versions [41, 43].

Since $U(N)=(S U(N) \times U(1)) / \mathbb{Z}_{N}$, we can in principle have different ChernSimons levels for the $S U(N)$ and $U(1)$ symmetries. We will denote a $U(N)$ Chern-Simons theory with levels $k_{1}$ and $k_{2}$ corresponding to the $S U(N)$ and $U(1)$ symmetries, respectively, as a $U(N)_{k_{1}, k_{2}}$ theory. Prior to the inclusion of matter in our model, we state an important fact about these theories. Namely, that some choices of gauge groups and levels are dual to others. This is known as level-rank duality. Generalising this duality to include matter is the duality that we present below.

Before we proceed with the statement of the dualities, we need to make a brief statement about regularisation schemes. In the study of QFTs, couplings generally get renormalised and Chern-Simons theory is no exception. Different regularisation procedures make different aspects clearer or make different calculations simpler. The Fermi-Bose dualities are usually discussed in socalled Yang-Mills regularisation. This procedure is performed by integrating out the Yang-Mills dynamics, which leave a quantised level in the infrared limit. For more details on this calculation, the reader may consult Pisarski $\mathcal{E}$ Rao [169].

In this regularisation, the Fermi-Bose dualities are as follows [40]

$$
\begin{aligned}
& S U(N)_{k}+\text { scalars } \longleftrightarrow U(k)_{-N+\frac{N_{f}}{2},-N+\frac{N_{f}}{2}}+\text { fermions, } \\
& U(N)_{k, k}+\text { scalars } \longleftrightarrow S U(k)_{-N+\frac{N_{f}}{2}} \quad+\text { fermions, } \\
& U(N)_{k, k+N}+\text { scalars } \longleftrightarrow U(k)_{-N+\frac{N_{f}}{2},-N+\frac{N_{f}}{2}-k}+\text { fermions. }
\end{aligned}
$$




\section{BACKGROUND}

The scalars on the LHS of these equations are assumed to be tuned to a critical interacting point of the RG flow, known as the Wilson-Fisher (WF) fixed point [162], which we elaborated on in the previous section. Similarly to ParticleVortex duality, which maps elementary excitations to non-perturbative vortices, Fermi-Bose duality maps the fundamental perturbative states on one side to monopole or baryon operators on the other side. A monopole operator $\mathcal{M}(x)$ is defined as inserting a unit of flux at a point $x$. This means that in the path integral we integrate over configurations with a fixed flux emanating from the point $x$

$$
\langle\mathcal{M}(x)\rangle=\left.\int \mathcal{D} A_{\mu} e^{i S}\right|_{\frac{1}{4 \pi} \int_{\mathrm{S}^{2}} d^{2} S_{\mu} \epsilon^{\mu \nu \rho} F_{\nu \rho}=1},
$$

where $\mathbf{S}^{2}$ is a sphere surrounding the monopole insertion point.

Understanding the details of this duality subject to the inclusion of a chemical potential has been the formative motivation for this work. We believe that some of the results of this thesis have sown the seed for this understanding. More specifically, in Chapter (3) we discover a non-trivial ground state that owes its existence to the non-zero value of the chemical potential. The role of this ground state in the duality still remains mysterious. The resolution of this mystery is left for future work. Thus we conclude our discussion of Fermi-Bose duality and move on to the physics of non-commutative fluids.

\subsection{Non-Commutative Fluids}

In this section we will define important physical notions in a non-commutative (NC) geometry and demonstrate how the non-commutative version of the Landau problem leads directly to the non-commutative Chern-Simons action. Finally, we discuss the connection of the Fock space of this action with the results that will be presented in Chapter (3). For a good summary of this topic, we refer the reader to this set of excellent reviews on physics on non-commutative geometry [82-84]. 
Non-commutative geometry can arise naturally from the quantisation of a particle's phase space coordinates. The simplest way to see this connection is through the Heisenberg algebra of the canonical commutation relations

$$
[\hat{x}, \hat{p}]=i \hbar
$$

As is well known, this quantisation condition leads to a "smearing out" of the phase space structure of the theory. This smearing makes the notion of a point in phase space non-sensical, since one can no longer measure both position and momentum accurately.

However, there are more exotic cases, where it is the coordinate space that becomes non-commutative or fuzzy, not the phase space. This is due to the specific form of the Poisson brackets in the problem. In such a problem, the notion of a point in space is no longer reasonable and one can no longer measure the position of a (quasi)particle to arbitrary accuracy on both axes at once. An example of a problem, where the different momenta exhibit such non-commutativity, is the problem of particles in a magnetic field that we discussed earlier. Similarly, we all know that angular momentum also behaves in a non-commutative way, so the idea of the spatial coordinates having a nonzero commutator comes naturally. Finally, in the study of fluids, one can define a type of Poisson bracket that is restricted to the coordinate space, which leads to a non-commutative space following a canonical quantisation [85].

The ideas of a non-commuting space-time were first explored by Snyder in 1947 [86, 87]. The subject of non-commutative geometry was first formalised by Connes in 1994 [88]. The first consideration of non-commutative geometry in the context of bosonic string theory and BRST ${ }^{1}$ quantisation was made by Witten [89] for open strings. This was later extended to closed bosonic strings by Sen $\mathcal{E}$ Holman [90]. Witten $\mathcal{E}$ Seiberg's seminal paper on non-commutative geometry in string theory shows that the motion of strings in a background gauge field is equivalent to a non-commutative Yang-Mills theory [91]. The presence of a background gauge field in this construction is reminiscent of the

\footnotetext{
${ }^{1}$ BRST stands for the names of the authors Becchi-Rouet-Stora-Tyutin who first came up with this approach to quantising gauge theories. [170-172]
} 
Hall effect. It turns out that non-commutative field theories are intricately linked with the physics of the Hall effect.

The first proposal that non-commutative Chern-Simons theory has something to do with the fractional quantum Hall effect came from Susskind [92]. He showed that abelian non-commutative Chern-Simons theory at level $k=n$ is exactly equivalent to the Laughlin theory at filling fraction $\frac{1}{n}$. Note here that the combination of adjectives "abelian" and "non-commutative" is not an oxymoron, since it is the space-time coordinates that do not commute, not the gauge fields. It can be shown that the level of non-commutative CS is also quantised [173, 174]. Relations between string theory and the quantum Hall effect have been established through non-commutative field theory [175-177]. A review on the applications of non-commutative field theory to physics has been compiled by Banerjee et al. [178]. Now that we have established the relevance of non-commutative geometry, we review some basic facts about it.

\subsubsection{Review of non-commutative geometry}

Let us see how to define a non-commutative space. Here we will be concerned with a $D$ dimensional space-time that has both a set of $D-2 p$ commuting $\left\{y_{i}, i=2 p+1, \ldots, D\right\}$ and $2 p$ non-commuting $\left\{x_{\alpha}, \alpha=1, \ldots, 2 p\right\}$ coordinates. More precisely,

$$
\begin{gathered}
{\left[y_{i}, y_{j}\right]=0,} \\
{\left[x_{\alpha}, x_{\beta}\right]=i \theta_{\alpha \beta},}
\end{gathered}
$$

where $\theta$ is an anti-symmetric constant two-form. One can perform linear transformations on the coordinates $x_{\alpha}$ so that the matrix $\theta$ is in canonical block form

$$
\theta_{\alpha \beta}=\theta\left[\begin{array}{lll}
i \sigma_{2} & & 0 \\
& \ddots & \\
0 & & i \sigma_{2}
\end{array}\right]
$$


where $\theta$ is called the non-commutativity parameter and

$$
i \sigma_{2}=\left[\begin{array}{cc}
0 & 1 \\
-1 & 0
\end{array}\right]
$$

is the second Pauli spin matrix. This leaves us with $p$ pairs of the Heisenberg algebra

$$
\left[x_{2 \alpha-1}, x_{2 \alpha}\right]=i \theta, \quad \alpha=1, \ldots, p .
$$

This quantisation of the spatial coordinates leads to the existence of a Hilbert space. Since we can now think of the coordinates as operators, each operator has a set of eigenstates with associated quantum numbers. To make this more explicit, let us define the creation, annihilation and number operators

$$
\begin{gathered}
\boldsymbol{a}_{\alpha}=\frac{x_{2 \alpha-1}+i x_{2 \alpha}}{\sqrt{2 \theta}}, \quad \boldsymbol{a}_{\alpha}^{\dagger}=\frac{x_{2 \alpha-1}-i x_{2 \alpha}}{\sqrt{2 \theta}}, \\
\boldsymbol{n}_{\alpha}=\boldsymbol{a}_{\alpha}^{\dagger} \boldsymbol{a}_{\alpha} .
\end{gathered}
$$

These operators allow us to build the familiar Fock space from QFT by acting on a vacuum state

$$
\left|n_{1}, \ldots, n_{p}\right\rangle=\boldsymbol{a}_{1}^{\dagger n_{1}} \ldots \boldsymbol{a}_{p}^{\dagger n_{p}}|0\rangle
$$

Further, we define the derivative operators through the relations

$$
\partial_{\mu} \cdot x_{v}=\left[\partial_{\mu}, x_{v}\right]=\delta_{\mu v}, \quad \mu, v=1, \ldots, D
$$

Specifically for the non-commuting coordinates, the form of $\partial_{\alpha}$ can be expressed as

$$
\partial_{\alpha}=-i \omega_{\alpha \beta} x_{\beta}, \quad \alpha, \beta=1, \ldots, 2 p,
$$

where $\omega_{\alpha \beta}=\left(\theta^{-1}\right)_{\alpha \beta}$. From here it follows that

$$
\left[\partial_{\alpha}, \partial_{\beta}\right]=i \omega_{\alpha \beta}
$$




\section{BACKGROUND}

We see that by promoting space-time coordinates into non-commuting variables, we can construct a Hilbert space of states and re-express concepts that deal with space-time into concepts that have to do with the abstract space of states. One can go further and define functions on this non-commutative space. This would lead to fields defined on a non-commutative geometry.

\subsubsection{Non-commutative field theory}

Now that we understand some of the basic ideas of non-commutative geometry, we would like to define a field theory on this non-commutative space. More specifically, we would be interested in a gauge theory, since our main subject of interest is Chern-Simons theory. The simplest possible gauge theory contains just a gauge field $A_{\mu}$. Further, the easiest way to construct a gauge invariant theory is by forming an action that is composed of a trace of covariant derivatives

$$
D_{\mu}=-i \partial_{\mu}+A_{\mu}
$$

since under a gauge transformation $D_{\mu}$ transforms as

$$
D_{\mu} \rightarrow U D_{\mu} U^{-1}
$$

Restricting ourselves to the non-commuting submanifold $(\mu=\alpha)$, we have

$$
D_{\alpha}=-i \partial_{\alpha}+A_{\alpha}=\omega_{\alpha \beta} x^{\beta}+A_{\alpha}=\omega_{\alpha \beta}\left(x^{\beta}+\theta^{\beta \gamma} A_{\gamma}\right) \equiv \omega_{\alpha \beta} X^{\beta}
$$

Let us now see how non-commutative Chern-Simons theory arises as the action of a massless particle confined to the plane in the presence of a magnetic field. Here we show that the physics of charged particles, confined to a plane and subject to a magnetic field, is linked to abelian Chern-Simons gauge theory [179, 180]. Then we show that the non-commutative generalisation of this model yields a lot of the distinct physical features of the FQHE [93] Let us take a look at the action of a particle of charge $q$ and mass $m$ moving in an 
electromagnetic field generated by the four-potential $A^{\mu}=(-V(\boldsymbol{x}, t), \boldsymbol{A}(\boldsymbol{x}, t))$

$$
S=\int d t\left(\frac{1}{2} m^{2} v^{2}-q V(\boldsymbol{x}, t)+q v \cdot \boldsymbol{A}(\boldsymbol{x}, t)\right)
$$

Now, let us assume that the particle is massless and, in addition, it is in a constant magnetic field and the electric potential is 0 . Since $\nabla \times \boldsymbol{A}=\boldsymbol{B}$, this implies that

$$
A_{i}=\frac{B}{2} \epsilon_{i j} x_{j}
$$

up to a gauge transformation, where $B=|B|$. Substituting Equation 1.169) into Equation (1.168), we arrive at the action

$$
S=\int d t\left(q \frac{B}{2} \epsilon_{i j} \dot{x_{i}} x_{j}\right) .
$$

From here we proceed by making the identification

$$
x_{i} \leftrightarrow X_{i}
$$

And since the $X_{i}$ fields transform under gauge transformations, we ought to make sure that the action remains invariant. To this end, we need to also gauge the time derivative $\partial_{0} \rightarrow D_{0}=\partial_{0}+A_{0}$ so that the action becomes

$$
S=\int d t\left[q \frac{B}{2} \epsilon_{i j} \operatorname{Tr}\left(D_{0} X^{i} X^{j}\right)\right]=\int d t\left[q \frac{B}{2} \epsilon_{i j} \operatorname{Tr}\left(\partial_{0} X^{i} X^{j}+\left[A_{0}, X^{i}\right] X^{j}\right)\right] .
$$

The introduction of the time component of the gauge field leads to a Gauss's law constraint

$$
\left[X^{1}, X^{2}\right]=0
$$

It seems that this constraint has removed the non-commutativity that we tried to introduce into our model. In order to bring it back, we add an extra term to the action such that we get the Heisenberg algebra 1.155 that we explored 
earlier

$$
S=\int d t\left[q \frac{B}{2} \epsilon_{i j} \operatorname{Tr}\left(\partial_{0} X^{i} X^{j}+\left[A_{0}, X^{i}\right] X^{j}+2 \theta A_{0}\right)\right] .
$$

Thus we have arrived at the non-commutative abelian Chern-Simons model by considering the movement of charged particles in a magnetic field confined to a two-dimensional plane.

We shall see in Chapter (3) how this non-commutative theory arises out of the ground state of a commutative non-abelian finite chemical potential Chern-Simons-matter theory. More specifically, the ground state matrices in our model form an algebra, which coincides with the algebra of the $X^{1}$ and $X^{2}$ coordinates, together with a Hamiltonian-like operator corresponding to the system (1.174). We hope that this unexpected link between NC abelian field theory and commutative non-abelian field theory would provide a bridge that will allow us to solve the non-abelian theory.

We have now covered all of the essential background material so we are ready to move on to our first set of results, which have to do with the physics of vortices in Chern-Simons-matter theory. This is what the next chapter is be about. 


\section{Chapter 2}

\section{Abelian Chern-Simons Vortices at Finite Chemical Potential}

\subsection{Introduction}

Chern-Simons theory and the dynamics of vortices are both intimately connected to the quantum Hall effect [143]. The Chern-Simons interaction has the effect of attaching a magnetic flux to a charged particle, and endowing magnetic flux carrying vortices with electric charge. Magnetic flux attachment changes the statistics of particles, so that fermions can be described as bosons with a Chern-Simons interaction. In particular, the effective theory of the fractional quantum Hall state is a complex scalar interacting with an abelian Chern-Simons gauge field [48, 143]. Vortex solitons in the relativistic abelian Higgs model in 2+1 dimensions in the presence of a Chern-Simons action (with or without a Maxwell term) have been extensively studied [46, 61-64, 181].

In this chapter we are interested in vortex solitons appearing in relativistic scalar field theory coupled to an abelian Chern-Simons gauge field when a chemical potential for particle number is turned on. Our motivation is to investigate vortex configurations whose presence is triggered purely by finite density effects in Chern-Simons-matter theories. The abelian Chern-Simonsscalar system offers the simplest such setting. Eventually, we would like to understand finite density vortex solutions in $S U(N)$ and $U(N)$ Chern-Simonsscalar theories where finite chemical potential results in condensation of gauge 


\section{ABELIAN CHERN-SIMONS VORTICES AT FINITE CHEMICAL POTENTIAL}

fields, potentially breaking rotational invariance [182 $]^{1}$. A broader aim for exploring different aspects of finite density physics in Chern-Simons-matter theories is to understand the implications of the associated web [74, 185, 186] of particle-vortex and Bose-Fermi dualities in 2+1 dimensions [39, 40, 42, 43, 81, 105, 187-189.

A chemical potential $\mu$ for a gauged $U(1)$ symmetry can only be turned on provided a source term representing a classical (frozen out) uniform background charge density is simultaneously introduced. In Chern-Simons theory, such a source can either be viewed as a distribution of heavy charges or as a uniform magnetic field. We focus on the massless scalar field, whose interaction potential is purely a power law

$$
U=\frac{g_{2 s}}{s}|\Phi|^{2 s}, \quad s=2,3, \ldots,
$$

with no symmetry breaking minima in vacuum, and solve the equations of motion, with non-zero $\mu$, numerically. Chern-Simons vortices with symmetry breaking potentials in vacuum have vanishing magnetic fields in the interior and on the outside, with the flux being supported at the edges. The finite $\mu$ vortex solutions are qualitatively different as the magnetic flux acquires support within the vortex interior, and depending on the sign of the quantised flux $(=2 \pi n)$, there are two qualitatively distinct types of solutions: (i) Those with negative flux, given our choice of conventions $(\mu>0$ and Chern-Simons level $k>0$ ), where the majority of the flux resides in the vortex interior, and (ii) positive flux solutions wherein most of the flux sits at the edge of the vortex. The qualitatively different behaviour of configurations with winding numbers $n<0$ and $n>0$ is expected due to the breaking of charge conjugation symmetry when $\mu \neq 0$. Positive flux solutions are energetically disfavoured, or more precisely the grand potential for the $n$-vortex with $n>0$ is parametrically larger, as a function of $n$, than that for the $n<0$ vortex.

\footnotetext{
${ }^{1}$ An analogous situation in $3+1$ dimensional Yang-Mills-Higgs system with $S U(2) \times U(1)$ gauge group was encountered by Miransky, Gusynin \& Shovkovy [183] where the finite density ground state breaks spatial isotropy due to condensation of vector fields. Vortex solutions in the condensed phase were subsequently found Miransky, Gorbar E Jia [184].
} 
The negative flux solutions are the most interesting. We find these numerically for a wide range of winding numbers, $1 \leq|n| \lesssim 10^{5}$. Supported by simple analytical arguments, we confirm that for large $|n|$, negative flux vortices for power law potentials 2.1 with general $s$ exhibit linear scaling of the grand potential with $|n|$ :

$$
\mathcal{E}(|n| \gg 1)=\frac{s-1}{2 s} k \mu|n| .
$$

For a specific value of the dimensionless coupling, the solutions are "BPS" or marginally bound:

$$
\left.\frac{\mathcal{E}(|n|)}{|n| \mathcal{E}(1)}\right|_{\alpha=s /(s-1)} \rightarrow 1, \quad \alpha \equiv \frac{k}{4 \pi}\left(\frac{g_{2 s}}{\mu^{3-s}}\right)^{1 /(s-1)}
$$

This critical value of $\alpha$ works surprisingly well even for low $n$ vortices. Below this value individual vortices experience attractive interactions, and repulsive interactions above it. At the critical coupling, we find numerically that the vortex profiles closely (but not exactly) solve the first order Bogomolny'i type equation. Finally, the radius of the $n$-vortex in all cases is given by

$$
\left.R_{n}\right|_{|n| \gg 1}=\sqrt{2 \alpha|n|} \mu^{-1}
$$

implying that the $n$-vortex behaves like a uniform incompressible droplet within which individual vortices are as closely packed as possible. The physical properties we have described closely resemble the non-relativistic supersymmetric Chern-Simons theory introduced in [190]. The scalings with $n$ are in line with the "MIT bag" model for solitons with large winding number, advocated in a series of publications by Bolognesi et al. [191-193], in which the vortices are thought of as a region of non-zero energy density separated from the outside via a domain wall. This is similar to the original MIT bag model named after the institution which the creators were associated with [194]. In this model hadrons are thought of as a confining region of space with non-zero energy density, within which the fermion and gluon fields are weakly interacting .

This chapter is organised as follows: In Sections (2.2) and 2.3), we review the standard vortex equations of motion, but in the presence of chem- 


\section{ABELIAN CHERN-SIMONS VORTICES AT FINITE CHEMICAL POTENTIAL}

ical potential. We also point out some features of the finite density spectrum and qualitative aspects of vortex profiles. In Section (2.4), we discuss the energy functional or the grand potential, and argue its expected scaling for large winding numbers. In Section 2.5), we present several results of the numerical analysis of the vortex equations of motion for different choices of potentials and parameters.

\subsection{The Abelian Theory at Finite Chemical Poten- tial}

Our starting point is the abelian Chern-Simons theory at level $k$ coupled to a relativistic scalar field in $2+1$ dimensions. As is customary, we may regard this system as the infrared limit of the abelian Higgs model with a MaxwellChern-Simons gauge field, since the Maxwell action is irrelevant compared to the Chern-Simons term. We want to consider the theory in the grand canonical ensemble with a chemical potential for the $U(1)$ charge. Turning on a chemical potential for a local symmetry is a subtle issue since the Gauss constraint requires the total charge in the system to vanish. This putative obstacle can be overcome by introducing a uniform external classical charge density which can be viewed as a distribution of heavy charged species whose fluctuations are frozen out [195, 196]. In the presence of a Chern-Simons density this can also conveniently be viewed as a constant background magnetic field.

The $U(1)$ chemical potential is introduced as usual via a constant temporal background gauge field. Picking a $(-++)$ metric signature, the Lagrangian density for the system is,

$$
\begin{aligned}
& \mathcal{L}=\mathcal{L}_{\text {matter }}+\mathcal{L}_{C S}-J_{0} A_{0}, \\
& \mathcal{L}_{\text {matter }}=D_{v} \Phi^{\dagger} D^{v} \Phi+U\left(\Phi^{\dagger} \Phi\right), \\
& \mathcal{L}_{C S}=\frac{k}{4 \pi} \epsilon^{v \lambda \sigma} A_{v} \partial_{\lambda} A_{\sigma} .
\end{aligned}
$$


The gauge-covariant derivatives on the complex scalar $\Phi$ include a background value $\mu$ for $A_{0}$, which is identified as a chemical potential for the $U(1)$ charge:

$$
D_{v} \equiv \partial_{v}-i A_{v}-i \mu \delta_{v, 0},
$$

and $J_{0}$ is the background classical charge density. We assume a simple power law potential:

$$
U\left(\Phi^{\dagger} \Phi\right)=\frac{g_{2 s}}{s}|\Phi|^{2 s}, \quad s \geq 2 .
$$

The cases of quartic $(s=2)$ and sextic $(s=3)$ potentials are special since they correspond to relevant and marginal interactions. Our main interest is in monotonic potentials with a global minimum at $\Phi=0$ (in the absence of a chemical potential), so that any stable vortex configurations only appear at finite density, i.e. they are driven by scalar condensation in the presence of the chemical potential.

The quartic potential is of general interest because when $\mu$ vanishes, the theory flows to the $2+1$ dimensional Wilson-Fisher fixed point coupled to a Chern-Simons gauge field. The critical scalar plays an important role in particle-vortex and the related web of Bose-Fermi dualities in $2+1$ dimensions [40]. Semi-classical solutions are far removed from this critical point and only reliable when $\mu / g_{4} \gg 1$.

The Lagrangian density expanded to show the $\mu$-dependent terms is,

$$
\begin{aligned}
\mathcal{L} & =\partial_{\nu} \Phi^{\dagger} \partial^{v} \Phi+i A_{v}\left(\Phi^{\dagger} \partial^{v} \Phi-\partial^{v} \Phi^{\dagger} \Phi\right)+A_{v} A^{v}|\Phi|^{2}+\frac{g_{2 s}}{s}|\Phi|^{2 s}-\mu^{2}|\Phi|^{2} \\
& +i \mu\left(\Phi^{\dagger} \partial^{0} \Phi-\partial^{0} \Phi^{\dagger} \Phi\right)-2 \mu A_{0}|\Phi|^{2}+\frac{k}{4 \pi} \epsilon^{v \lambda \sigma} A_{v} \partial_{\lambda} A_{\sigma}-J_{0} A_{0}
\end{aligned}
$$

The background charge density $J_{0}$ is fixed by requiring that the expectation values of $A_{0}$ and the magnetic field vanish in the ground state:

$$
\left\langle A_{0}\right\rangle=0 \Longrightarrow J_{0}=-2 \mu\langle|\Phi|\rangle^{2}, \quad\langle|\Phi|\rangle=v=\left(\frac{\mu^{2}}{g_{2 s}}\right)^{\frac{1}{2 s-2}} .
$$

The ground state conditions are also solved by a vanishing source $J_{0}=0$, and an $A_{0}$ expectation value set by the chemical potential. This latter solution is 


\section{ABELIAN CHERN-SIMONS VORTICES AT FINITE CHEMICAL POTENTIAL}

equivalent to absorbing the chemical potential via a shift in the gauge field leaving the partition function unchanged. This possibility is excluded by imposing a vanishing $A_{0}$ at infinity as a boundary condition.

The classical source $J_{0}$ can also be interpreted as a background magnetic field. Defining

$$
B=\epsilon^{0 i j} \partial_{i} A_{j}
$$

and assuming a static configuration, the equation of motion for $A_{0}$ yields,

$$
J_{0}-\frac{k}{2 \pi}\langle B\rangle+2\left(\mu+\left\langle A_{0}\right\rangle\right) v^{2}=0 .
$$

Therefore, even if the source $J_{0}$ were not explicitly introduced, it is naturally induced through a non-zero background value for $B$. We will treat this background value as distinct from the magnetic field carried by the vortex.

\subsubsection{Perturbative spectrum}

The vacuum expectation value for $\Phi$ Higgses the gauge group and since ChernSimons gauge fields do not propagate, the physical perturbative spectrum consists of two gapped excitations. The dispersion relations can be found by expanding the gauge-fixed action $\bigsqcup^{1}$ to quadratic order in fluctuations and identifying the gauge-invariant zeros of the fluctuation determinant. Let the quadratic fluctuations be defined as follows:

$$
\Phi=\left(v+\varphi_{1}\right)+i \varphi_{2}, \quad A_{\mu}=\delta A_{\mu}
$$

\footnotetext{
${ }^{1}$ We use an $R_{\xi}$ gauge fixing term of the form $\mathcal{L}_{g f}=\left(\partial_{\mu} A^{\mu}-i\left(\xi\langle\Phi\rangle \delta \Phi^{\dagger}-\xi \delta \Phi\langle\Phi\rangle^{\dagger}\right)\right)^{2} /(2 \xi)$
} 
Then the quadratic Lagrangian of the fluctuations in phase space becomes

$$
\begin{aligned}
\mathcal{L}^{(2)} & =\left(\varphi_{1}^{2}+\varphi_{2}^{2}\right)\left(-\omega^{2}+\mathbf{p}^{2}-\mu^{2}\right)+g_{2 s} v^{2(s-1)}\left(\varphi_{2}^{2}+(2 s-1) \varphi_{1}^{2}\right) \\
& -4 i \mu \omega \varphi_{1} \varphi_{2}-4 v \mu \delta A_{0} \varphi_{1}+v^{2} \delta A_{\mu} \delta A^{\mu} \\
& -\frac{i k}{2 \pi}\left(\delta A_{0} p_{1} \delta A_{2}-\delta A_{0} p_{2} \delta A_{1}+\delta A_{1} \omega \delta A_{2}\right) \\
& -\frac{1}{2 \xi} p_{\mu} p_{v} \delta A^{\mu} \delta A^{v}-2 \xi v^{2} \varphi_{2}^{2} .
\end{aligned}
$$

From this expression we can extract the following matrix, the zero eigenvalues of which are the elementary excitations of the model,

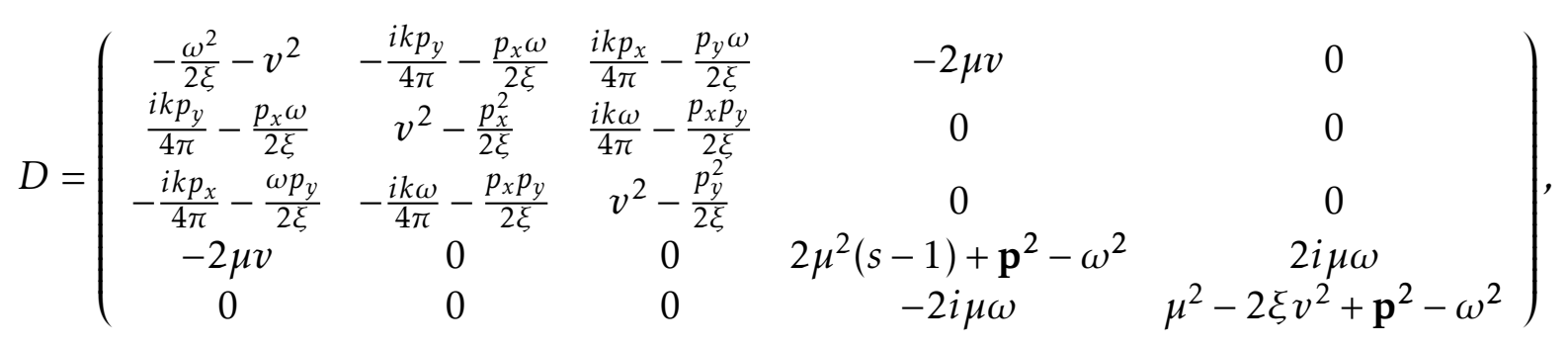

where we have used $g_{2 s}=\frac{\mu^{2}}{v^{2(s-1)}}$. Note that this is a $5 x 5$ matrix, but we stated earlier that the number of excitations in this model is only $d_{\mathrm{dof}}=2$, where $d_{\mathrm{dof}}$ is the number of physical degrees of freedom. The reason for this mismatch is that 3 out of the 5 solutions, that we get, are going to be gauge dependent. Once we remove the gauge dependent solutions, we will be left with the two physical excitations present in the spectrum of the theory.

One way of computing the elementary excitations is by finding all of the eigenvalues explicitly. This is not trivial in the general case so here we will illustrate an approach that can be used when symbolic computation of all the eigenvalues is unfeasible. This approach is also utilised in Chapter (3), but we outline it here since the calculation is simpler and more instructive. The way we can find the physical eigenvalues is by picking out the highest order term in $\xi$ in the determinant. Let us see why this works. We know that without gauge fixing, $D$ is non-invertible. First we express $D$ as the product of eigenvalues

$$
\operatorname{det}(D)=\lambda_{1} \ldots \lambda_{r}
$$




\section{ABELIAN CHERN-SIMONS VORTICES AT FINITE CHEMICAL POTENTIAL}

where $r$ is the size of the square matrix. Suppose, without loss of generality, that the physical eigenvalues are $\lambda_{1}$ and $\lambda_{2}$. Hence the dispersion relations correspond to $\lambda_{1}=0$ and $\lambda_{2}=0$. Then the determinant can be expressed as

$$
\operatorname{det}(D)=\lambda_{1} \lambda_{2}\left(\sum_{i_{1}=-p_{1}}^{q_{1}} a_{i_{1}}^{(1)} \xi^{i_{1}}\right) \ldots\left(\sum_{i_{r-2}=-p_{r-2}}^{q_{r-2}} a_{i_{r-2}}^{(r-2)} \xi^{i_{r-2}}\right),
$$

where $p_{1}, p_{2}, \ldots, p_{r-2}, q_{1}, q_{2}, \ldots, q_{r-2} \in \mathbb{N}$. Multiplying out the $\xi$ dependent sums and setting the determinant to 0 we arrive at

$$
\operatorname{det}(D)=\lambda_{1} \lambda_{2} \sum_{i=-p}^{q} a_{i} \xi^{i}=0
$$

where $p, q \in \mathbb{N}$. Assuming $\xi \neq 0$, we divide by $\xi^{q}$ and take the $\xi \rightarrow \infty$ limit

$$
\lambda_{1} \lambda_{2} a_{q}=0
$$

Solving 2.17 for the energy $\omega$ gives us a number of solutions. If this number matches $d_{\text {dof }}$ exactly, then our job is done and we have found the correct dispersion relations. If, however, this number is larger than $d_{\text {dof }}$ that the system has, it means that we have stumbled upon a gauge mode that needs to be removed. We can plug in the solutions we have found into the next to leading order term in $\xi$, namely

$$
\lambda_{1} \lambda_{2} a_{q-1}
$$

If the number of common solutions to these two equations is $d_{\text {dof }}$, we have found the physical dispersion relations. In principle, one can continue this procedure order by order in $\xi$ for all of the coefficients. If the gauge procedure is done properly, we are guaranteed to find the physical solutions, since if we have more than $d_{\text {dof }}$ dispersion relations that are independent of $\xi$, this means that there is a gauge mode that is independent of $\xi$, but that is impossible since those modes would return an identically zero eigenvalue prior to gauge fixing.

The dispersion relations for the two physical modes can be expressed in 
terms of dimensionless frequency and momentum and a single dimensionless

coupling (assuming $k>0, \mu>0$ )

$$
\alpha \equiv \frac{k \mu}{4 \pi v^{2}}, \quad \tilde{\omega} \equiv \omega / \mu, \quad \tilde{\mathbf{p}} \equiv \mathbf{p} / \mu
$$

We find,

$$
\tilde{\omega}_{ \pm}=\sqrt{\tilde{\mathbf{p}}^{2}+(s+1)+\frac{1}{2 \alpha^{2}} \pm \sqrt{4 \tilde{\mathbf{p}}^{2}+\left(s+1-\frac{1}{2 \alpha^{2}}\right)^{2}}} .
$$

Both modes are gapped at $\tilde{\mathbf{p}}=0$, as can be seen here

$$
\begin{array}{ll}
\tilde{\omega}_{+}^{2} \simeq 2(s+1)+\tilde{\mathbf{p}}^{2} \frac{2 \alpha^{2}(s+3)-1}{2 \alpha^{2}(s+1)-1} \ldots, & \\
\tilde{\omega}_{-}^{2} \simeq \frac{1}{\alpha^{2}}+\tilde{\mathbf{p}}^{2} \frac{2 \alpha^{2}(s-1)-1}{2 \alpha^{2}(s+1)-1} \ldots, \quad|\tilde{\mathbf{p}}| \ll 1 .
\end{array}
$$

When the Chern-Simons level is taken to be large the mode $\tilde{\omega}_{-}$is the lighter of the two and becomes the phonon in the strict $k \rightarrow \infty$ limit. For the classically marginal sextic potential with $s=3$, this limit yields $\tilde{\omega}_{-}^{2} \simeq \tilde{\mathbf{p}}^{2} / 2$ which implies a speed of sound $c_{s}=1 / \sqrt{2}$, expected from scale invariance in $2+1$ dimensions. As the level $k$ is lowered, the gaps of the two branches coincide when $\alpha=$ $1 / \sqrt{2(s+1)}$, and below this value of $\alpha$, the roots exchange roles. 


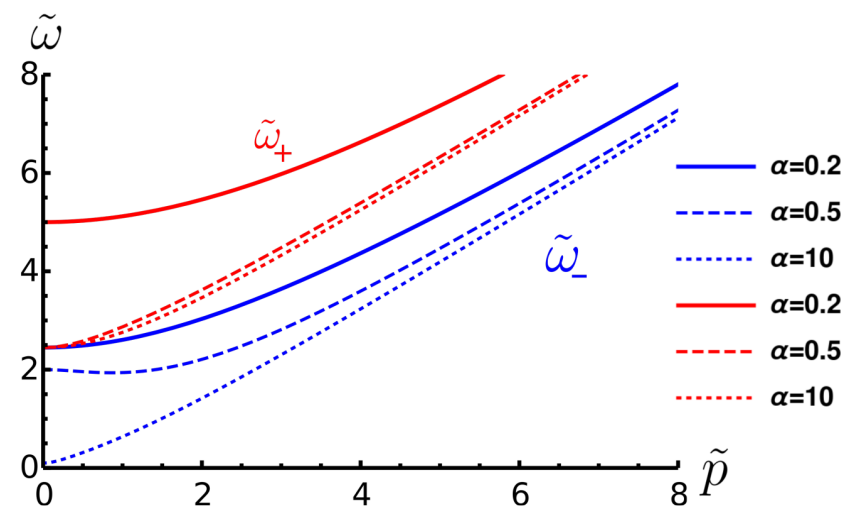

Figure 2.1: The dispersion relations of the two branches of perturbative fluctuations for the quartic potential $(s=2)$, with different values of the dimensionless coupling $\alpha$.

Depending on the value of $\alpha$, the sign of $\tilde{\omega}_{ \pm}^{\prime \prime}(0)$ can be negative which implies a minimum in the dispersion relation away from $\tilde{\mathbf{p}}=0$, also called a magneto-roton minimum. One of the two branches will exhibit a magnetoroton minimum for values of $\alpha$ in the range:

$$
\frac{1}{\sqrt{2(s+3)}}<\alpha<\frac{1}{\sqrt{2(s-1)}}
$$

In the case of $s=2$, the roton range becomes $\frac{1}{\sqrt{10}}<\alpha<\frac{1}{\sqrt{2}}$, and as can be seen from Fig (2.1), $\tilde{\omega}_{-}$has a roton minimum for $\alpha=\frac{1}{2}$. This is not the case for the other plotted values of $\alpha$.

\subsection{Vortex Equations}

We want to solve the equations of motion in polar coordinates. Therefore, allowing for a non-trivial spatial metric $h$, the static field equations are:

$$
\begin{aligned}
& \frac{1}{\sqrt{h}} \partial_{j}\left(\sqrt{h} \partial^{j} \Phi\right)-\frac{i}{\sqrt{h}} \partial_{j}\left(\sqrt{h} A^{j} \Phi\right)+\left(\mu+A_{0}\right)^{2} \Phi-i A_{j} \partial^{j} \Phi-A_{j} A^{j} \Phi=g_{2 s}|\Phi|^{2 s-2} \Phi, \\
& \frac{k}{2 \pi} \epsilon^{\sigma v \rho} \partial_{v} A_{\rho}=-\sqrt{h}\left[i\left(\partial^{\sigma} \Phi \Phi^{+}-\Phi \partial^{\sigma} \Phi^{\dagger}\right)+2 A^{\sigma}|\Phi|^{2}-\delta_{0 \sigma}\left(2 \mu|\Phi|^{2}+J_{0}\right)\right] .
\end{aligned}
$$


As usual, a static vortex configuration carrying $n$ units of magnetic flux is described by the rotationally symmetric ansatz in polar coordinates:

$$
A_{0}=A_{0}(r), \quad A_{r}=0, \quad A_{\theta}=A_{\theta}(r), \quad \Phi=f(r) e^{i n \theta}
$$

This ansatz leads to the following system of equations ${ }^{1}$,

$$
\begin{aligned}
& f^{\prime \prime}+\frac{f^{\prime}}{r}-\frac{1}{r^{2}}\left(A_{\theta}-n\right)^{2} f+\left(A_{0}+\mu\right)^{2} f-g_{2 s} f^{2 s-1}=0, \\
& -\frac{f^{2}}{r}\left(A_{\theta}-n\right)+\frac{k}{4 \pi} A_{0}^{\prime}=0, \\
& -f^{2} A_{0}-\mu f^{2}+\frac{k}{4 \pi}\left(\frac{A_{\theta}^{\prime}}{r}\right)=\frac{J_{0}}{2}, \quad J_{0}=-2 \mu v^{2} .
\end{aligned}
$$

We are looking for a configuration which asymptotes at infinity to the ground state with the fixed non-vanishing background value for $J_{0}$, obeying the boundary conditions:

$$
A_{\theta}(r) \stackrel{r \rightarrow \infty}{\longrightarrow} n, \quad A_{0}(r) \stackrel{r \rightarrow \infty}{\longrightarrow} 0, \quad f(r) \stackrel{r \rightarrow \infty}{\longrightarrow} v, \quad f(0)=0 .
$$

With the rotationally symmetric ansatz above, the magnetic field $B$ only depends on $r$, and the configuration carries $n$ units of flux:

$$
B(r)=\frac{A_{\theta}^{\prime}(r)}{r}, \quad \Phi_{B}=\lim _{r \rightarrow \infty} \int_{0}^{2 \pi} A_{\theta}(r) d \theta=2 \pi n,
$$

assuming $A_{\theta}$ vanishes at the origin.

\subsubsection{Qualitative features}

The equation of motion 2.27) for $A_{0}$, which implements the Gauss constraint, fixes the value of the magnetic field at the core of the vortex where the scalar field vanishes. Thus,

$$
B(0)=\frac{4 \pi}{k} J_{0}=-\frac{\mu^{2}}{\alpha},
$$

\footnotetext{
${ }^{1}$ We omit the equation of motion for $A_{r}$ which is automatically satisfied.
} 


\section{ABELIAN CHERN-SIMONS VORTICES AT FINITE CHEMICAL POTENTIAL}

where $\alpha$ is the effective coupling defined in 2.19). For a given winding number $n$, the value of $\alpha$ completely characterises the vortex solution when expressed in terms of rescaled dimensionless variables. The effect of the Gauss constraint is to induce a magnetic field $B$ inside the vortex core to precisely cancel the background source $J_{0}$ which could also be viewed as a uniform background magnetic field. Outside the vortex $B$ vanishes,

$$
\lim _{r \rightarrow \infty} B(r)=0
$$

The sign of $B(0)$ plays an important role in determining the properties of the vortex solutions. Without loss of generality, we assume that the chemical potential $\mu$ and the Chern-Simons level $k$ are both positive:

$$
\mu>0, \quad k>0
$$

With this choice $B(0)$ is negative definite, independently of the sign of the magnetic flux $2 \pi n$. However, this means that solutions with positive and negative flux will be qualitatively different. This breaking of charge conjugation is precisely what we expect in the presence of the $U(1)$ chemical potential.

Negative flux $n<0$ : Assuming that the magnitude of the magnetic field $|B(r)|$ increases monotonically towards the vortex core, and given that the value of the core magnetic field is independent of $|n|$ (the number of units of flux) the vortex core size should increase with $|n|$ for negative $n$. Taking $B$ to be uniform within the core for large enough $|n|$, we can estimate the radius $R_{n}$ of a vortex solution with $|n| \gg 1$ :

$$
|B(0)| \pi R_{n}^{2} \approx 2 \pi|n| \quad \Longrightarrow \quad R_{n} \approx \frac{\sqrt{2 \alpha|n|}}{\mu}, \quad|n| \gg 1
$$

The assumption of uniformity of the vortex core region is self-consistently justified by first noting that for small $r$,

$$
f(r)=c_{0} r^{|n|}+\ldots, \quad c_{0}>0 .
$$


This is obtained by neglecting $A_{\theta}$ in comparison to $n$, and ignoring higher order terms for small $r$ in Equation 2.25). Therefore, for large flux, the scalar profile is extremely flat near $r=0$. With a uniform $B$ field in the vortex interior, the vector potential is determined as

$$
A_{\theta} \approx-\frac{1}{2}|B(0)| r^{2},
$$

and this approximation breaks down precisely when $r \approx R_{n}$ (see Equation (2.33) ) . We see numerically that the scalar field profile, inside the vortex, closely follows the solution to the first order equation:

$$
\begin{aligned}
f^{\prime}(r) & \approx \frac{1}{r}\left(|n|-\frac{1}{2}|B(0)| r^{2}\right) f(r) \\
\Longrightarrow \quad f(r) & \approx c_{0} r^{|n|} e^{-|B(0)| r^{2} / 4}, \quad r<R_{n} .
\end{aligned}
$$

This feature of the solution is depicted in Figure 2.6

The equation of motion (2.26) for $A_{\theta}$ determines the radial electric field $E(r)=A_{0}^{\prime}(r)$. The electrostatic potential $A_{0}$ remains constant inside the core region since $f(r)$ is vanishingly small and therefore the electric field is also vanishingly small. Outside the core region the electric and magnetic fields decay exponentially to zero. Linearising about the asymptotic solution at large $r$ we find,

$$
\begin{aligned}
& \delta f \equiv f(r)-v, \quad \delta A_{\theta}=A_{\theta}-n, \\
& \delta A_{\theta}=\frac{\alpha}{\mu} r A_{0}^{\prime}, \\
& \delta f^{\prime \prime}+\frac{\delta f^{\prime}}{r}-(2 s-2) \mu^{2} \delta f=-2 A_{0} \mu v, \\
& A_{0}^{\prime \prime}+\frac{A_{0}^{\prime}}{r}-\frac{\mu^{2}}{\alpha^{2}} A_{0}=\frac{2 \mu^{3}}{v \alpha^{2}} \delta f .
\end{aligned}
$$

The solutions to the homogeneous equations for the fluctuations $\delta f$ and $A_{0}$ 


\section{ABELIAN CHERN-SIMONS VORTICES AT FINITE CHEMICAL POTENTIAL}

are the Bessel functions $K_{0}(\sqrt{2 s-2} \mu r)$ and $K_{0}(\mu r / \alpha)$ respectively, and these control the exponential decay of fluctuations at large $r$,

$$
\left.K_{0}(\sqrt{2 s-2} \mu r)\right|_{\mu r \rightarrow \infty} \sim \frac{e^{-\sqrt{2 s-2} \mu r}}{\sqrt{\mu r}},\left.\quad K_{0}(\mu r / \alpha)\right|_{\mu r \rightarrow \infty} \sim \frac{e^{-\mu r / \alpha}}{\sqrt{\mu r}}
$$

The two exponents are related to masses of the gapped perturbative excitations around the Higgsed ground state. Since $A_{\theta}(r) \simeq n$ outside the core region, according to Equation 2.26 the electric field is only significant in a strip along the edge of the vortex core. Numerically, we find that the width of this strip does not scale with $|n|$, so that in the limit of large $|n|$, the contribution from the transition region to the vortex energy is subleading in $|n|$.

We will see below that these qualitative aspects of the vortex solutions lead to linear dependence of the vortex energy on $|n|$ and BPS-like behaviour at a critical value of the effective coupling $\alpha$.

Positive flux $n>0$ : Positive flux solutions are qualitatively distinct from the negative flux ones. This is because $B(0)<0$ independently of $n$, so $B(r)$ must switch sign to yield a net positive flux. For large $n>0$, most of this positive flux remains concentrated in a ring-like region at the edge of the vortex. In this case the total flux can be written as a sum of two contributions, one that scales with the area of the vortex and is negative, therefore must be subleading, and a positive dominant contribution which scales with the radius of the configuration,

$$
2 \pi n \sim-\pi R_{n}^{2} \frac{\mu^{2}}{\alpha}+2 \pi R_{n} \Delta_{\text {ring }} B_{\text {ring }} .
$$

Here $\Delta_{\text {ring }}$ is the width of the edge region which we take to be independent of $n$, whilst $B_{\text {ring }}$ denotes the peak value of the magnetic field in the ring and $R_{n}$ is the radius of the vortex for large enough $n$. The negative area-dependent contribution is a key difference from a similar situation discussed by Bolognesi [193]. We cannot exclude the possibility that both the area and perimeter contributions have faster than linear growth and a delicate cancellation yields the correct flux. It is possible to suppress the area contribution by making $\alpha$ arbitrarily large. The large $\alpha$ limit makes the magnetic field inside the vortex vanishingly small and the system then closely resembles an abelian Chern-Simons 
vortex in vacuum. Conversely, a small value of $\alpha$ (or equivalently a large value of $\mu$ and a fixed $\alpha$ ) makes the magnetic field inside the vortex larger and forces the transition between the two vacua to be steeper, effectively approaching a step function domain wall solution, analogous to the MIT bag models discussed earlier.

\subsection{Vortex Energy and BPS-like Scaling}

The "energy" functional appropriate for the grand canonical ensemble is the grand potential. The Chern-Simons term does not contribute to it for static configurations. The grand potential is obtained by using the Lagrangian density 2.8 with $\mu \neq 0$ and passing to the Hamiltonian picture. Rewriting the Hamiltonian in terms of the fields and their derivatives, the desired energy functional is 1 ,

$$
\mathcal{E}=\int d^{2} x\left(\left|D_{i} \Phi\right|^{2}+A_{0}^{2}|\Phi|^{2}+\frac{g_{2 s}}{s}|\Phi|^{2 s}-\mu^{2}|\Phi|^{2}\right)
$$

accompanied by the constraint which incorporates the effect of the ChernSimons term,

$$
\frac{k}{4 \pi} B=A_{0}|\Phi|^{2}+\mu\left(|\Phi|^{2}-v^{2}\right)
$$

An interesting feature of the finite- $\mu$ vortices (with negative flux) is that they appear to be marginally bound (or BPS-like) for a specific value of the effective dimensionless parameter $\alpha$. Recall that this parameter depends on the Chern-Simons level, the chemical potential and the interaction strength:

$$
\alpha=\frac{k \mu}{4 \pi v^{2}}=\frac{k}{4 \pi}\left(\frac{g_{2 s}}{\mu^{3-s}}\right)^{\frac{1}{s-1}} .
$$

\footnotetext{
${ }^{1}$ For static configurations, the grand potential can be quickly derived by retaining only the spatial gradient and potential terms including the Chern-Simons density,

$$
\mathcal{E}=\int d^{2} x\left(\left|D_{i} \Phi\right|^{2}-\left(A_{0}+\mu\right)^{2}|\Phi|^{2}+A_{0}\left(\frac{k}{2 \pi} B-J_{0}\right)+\frac{g_{2 s}}{s}|\Phi|^{2 s}\right),
$$

and then applying the constraint equation 2.42.
} 


\section{ABELIAN CHERN-SIMONS VORTICES AT FINITE CHEMICAL POTENTIAL}

Let us perform a rescaling of variables and fields so that the equations of motion can be written explicitly in terms of dimensionless quantities:

$$
\tilde{r} \equiv \mu r, \quad \tilde{f} \equiv \frac{f}{v}, \quad a \equiv \frac{1}{\tilde{r}}\left(A_{\theta}-n\right), \quad \tilde{A}_{0} \equiv \frac{A_{0}}{\mu} .
$$

The rescaled scalar profile vanishes at $\tilde{r}=0$ and approaches unity for large $\tilde{r}$ : $\tilde{f}(\tilde{r} \rightarrow \infty)=1$. The asymptotic behaviours of $a(\tilde{r})$ and $\tilde{A}_{0}$ are,

$$
\lim _{\tilde{r} \rightarrow 0} \tilde{r} a(\tilde{r})=-n, \quad \lim _{\tilde{r} \rightarrow \infty} \tilde{r} a(\tilde{r})=0, \quad \lim _{\tilde{r} \rightarrow \infty} \tilde{A}_{0}(\tilde{r})=0 .
$$

The resulting dimensionless equations of motion (primes denote derivatives with respect to $\tilde{r}$ ) are,

$$
\begin{aligned}
& \tilde{f}^{\prime \prime}+\frac{\tilde{f}^{\prime}}{\tilde{r}}-a^{2} \tilde{f}+\left(\tilde{A}_{0}+1\right)^{2} \tilde{f}-\tilde{f}^{2 s-1}=0, \\
& \alpha \tilde{A}_{0}^{\prime}=a \tilde{f}^{2} \\
& \alpha \tilde{B}=\left(\tilde{f}^{2}-1\right)+\tilde{f}^{2} \tilde{A}_{0} .
\end{aligned}
$$

Therefore, for a fixed flux $n$, the solutions are only parametrised by the dimensionless effective coupling $\alpha$. Note that we have introduced the dimensionless magnetic field,

$$
\tilde{B}=\frac{1}{\tilde{r}}(\tilde{r} a)^{\prime}=\frac{A_{\theta}^{\prime}}{\tilde{r}} .
$$

We first rewrite the energy functional using the rescaled fields and variables,

$$
\mathcal{E}=2 \pi v^{2} \int_{0}^{\infty} \tilde{r} d \tilde{r}\left[\left(\tilde{f}^{\prime}-a \tilde{f}\right)^{2}+a \frac{d}{d \tilde{r}}\left(\tilde{f}^{2}-1\right)+\tilde{A}_{0}^{2} \tilde{f}^{2}+\frac{\tilde{f}^{2 s}}{s}-\tilde{f}^{2}+\frac{s-1}{s}\right]
$$

where we have included a constant zero-point shift so that the energy density is vanishing for the ground state at infinity. The second term in Equation 2.50) when integrated by parts yields a non-vanishing surface contribution,

$$
\int_{0}^{\infty} d \tilde{r}(\tilde{r} a) \frac{d}{d \tilde{r}}\left(\tilde{f}^{2}-1\right)=|n|-\int_{0}^{\infty} \tilde{r} d \tilde{r} \tilde{B}\left(\tilde{f}^{2}-1\right) .
$$


Employing the Gauss constraint to eliminate $\tilde{B}$ in favour of $\tilde{A}_{0}$, we obtain an expression for the energy functional which is suitable for subsequent approximations and matching with numerical results,

$$
\begin{gathered}
\mathcal{E}=2 \pi v^{2}|n|+2 \pi v^{2} \int_{0}^{\infty} \tilde{r} d \tilde{r} \quad\left[\left(\tilde{f}^{\prime}-a \tilde{f}\right)^{2}+\left(\frac{\tilde{f}^{2 s}}{s}-\tilde{f}^{2}+\frac{s-1}{s}\right)-\frac{1}{\alpha}\left(1-\tilde{f}^{2}\right)^{2}+\right. \\
\left.\tilde{A}_{0}^{2} \tilde{f}^{2}+\frac{1}{\alpha} \tilde{A}_{0} \tilde{f}^{2}\left(1-\tilde{f}^{2}\right)\right] .
\end{gathered}
$$

\subsubsection{The quartic potential $s=2$}

Anticipating numerical results in the next section, we can make certain observations concerning the energetics of vortex solutions for large negative flux.

Our arguments rely on the fact that for $n$ sufficiently large and negative, all fields are uniform inside and outside the vortex. Moreover, the vortex has a radius that scales as $\sqrt{|n|}$, and a thin transition region, whose width does not scale with $n$. In the case of the quartic potential the energy functional is,

$$
\begin{aligned}
\left.\mathcal{E}(n, \alpha)\right|_{s=2}=2 \pi v^{2}|n|+2 \pi v^{2} \int_{0}^{\infty} \tilde{r} d \tilde{r} \quad & {\left[\left(\tilde{f}^{\prime}-a \tilde{f}\right)^{2}+\left(\frac{1}{2}-\frac{1}{\alpha}\right)\left(1-\tilde{f}^{2}\right)^{2}+\right.} \\
& \left.+\tilde{A}_{0}^{2} \tilde{f}^{2}+\frac{1}{\alpha} \tilde{A}_{0} \tilde{f}^{2}\left(1-\tilde{f}^{2}\right)\right] .
\end{aligned}
$$

We know that $\tilde{f}$ vanishes inside the vortex, whilst $\tilde{A}_{0}$ and $a(r)$ vanish outside it. Specifically when $\alpha=2$, the scalar potential is precisely cancelled, and the integrand in the expression above has support only within the transition region at the edge of the vortex. If we assume that this contribution does not scale with $n$, we conclude that

$$
\left.\mathcal{E}(n, \alpha=2)\right|_{s=2,|n| \gg 1}=2 \pi v^{2}|n| .
$$

Our numerical solutions confirm (Figure 2.5) this conclusion which works remarkably well even for low values of $n$, including $|n|=1,2 \ldots$. Another surprising feature of the solutions for $\alpha=2$ is that they appear to solve the first order 


\section{ABELIAN CHERN-SIMONS VORTICES AT FINITE CHEMICAL POTENTIAL}

equation $\tilde{f}^{\prime}=a \tilde{f}$ to very high numerical accuracy (Figure 2.6.1.

It is easy to extend the arguments to other values of $\alpha$. When $\alpha \neq 2$, the second term in the integrand in Equation 2.53 provides an approximately constant energy density inside the vortex where $\tilde{f}=0$, while all other contributions remain vanishingly small. We therefore find,

$$
\left.\mathcal{E}(n, \alpha)\right|_{s=2,|n| \gg 1}=2 \pi v^{2}\left(|n|+\frac{1}{2} \mu^{2} R_{n}^{2}\left(\frac{1}{2}-\frac{1}{\alpha}\right)\right)=\alpha \pi v^{2}|n| .
$$

This behaviour is also confirmed numerically in Figure 2.5, albeit only for large $|n|$ as expected. Interestingly, although $v$ and $\alpha$ each depend on the quartic coupling constant $g_{4}$, the large $|n|$ vortex mass formula depends only on the Chern-Simons level $k$ and the chemical potential,

$$
\left.\mathcal{E}(n, \alpha)\right|_{s=2,|n| \gg 1}=\alpha \pi v^{2}|n|=\frac{k \mu}{4}|n| .
$$

\subsubsection{General power law potential $(s \geq 2)$}

The energies of solutions for general power law potentials now work along similar lines. The integrand for the energy density in Equation 2.52 is negligible both inside and outside the vortex when $\alpha=\alpha_{c}$,

$$
\alpha_{c}=\frac{s}{s-1}
$$

At this critical $\alpha$ we expect solutions with large flux to have energies $\mathcal{E}(n) \simeq$ $2 \pi v^{2}|n|$. When $\alpha$ takes generic values away from $\alpha_{c}$, adapting the $s=2$ argument to general power law potentials $\sim g_{2 s}|\Phi|^{2 s}$ we obtain,

$$
\left.\mathcal{E}(n, \alpha ; s)\right|_{|n| \gg 1}=2 \alpha \pi v^{2}|n| \frac{s-1}{s}=\frac{s-1}{2 s} k \mu|n| .
$$

This result is confirmed by our numerical solutions for the sextic potential below. As before the mass formula is independent of the interaction strength

\footnotetext{
${ }^{1}$ In this context, it is worth noting that if the terms proportional to $\tilde{A}_{0}$ are omitted (or assumed to be negligible) in Equations (2.46) and 2.48, then the resulting equations coincide, for a particular value of $\alpha$, with equations of motion for a BPS vortex in $U(2) \times U(2)$ ABJM theory [197] which solves an equivalent first order system [198].
} 
of the potential. We point out, however, that the radius of the vortex solution $R_{n}=\frac{1}{\mu} \sqrt{2 \alpha|n|}$ depends non-trivially on all parameters. From the definition of $\alpha$ (Equation (2.43)), increasing the interaction strength (for fixed $\mu$ and $k$ ) has the effect of increasing the vortex size.

\subsubsection{Positive flux vortices}

With our choice of conventions $\mu>0$ and $k>0$, positive flux solutions are energetically disfavoured. To see this, let us reconsider the energy functional 2.50. As in the case of the flux in Equation (2.39), there are different types of contributions to the energy, those that scale with the area of the vortex, those that scale with the perimeter, and finally (gauge-covariant) gradient terms which become important at the edge of the vortex. If we pick $\alpha$ to be sufficiently large so that we can ignore the flux contribution from the vortex interior, then $B_{\text {ring }} \sim n / R_{n}$. Then the leading contributions to the energy take the schematic form,

$$
\mathcal{E}(n) \sim\left(2 \pi v^{2}\right)\left(\frac{n^{2}}{R_{n}} \Delta+c_{0} \pi R_{n}^{2}\right), \quad \alpha \gg 1 .
$$

Here the first term is a perimeter contribution from a covariant gradient while the second term is a potential energy contribution from the interior. Extremising with respect to $R_{n}$, we obtain $R_{n} \sim n^{2 / 3}$ and $\mathcal{E}(n) \sim n^{4 / 3}$. However this argument will fail for finite $\alpha$ since $B(0)=-\mu^{2} / \alpha$ and the area scaling as $n^{4 / 3}$ would violate the flux condition in Equation 2.39). We have not found a satisfactory scaling argument for finite $\alpha$ and large $n$, but our numerical results indicate a faster than quadratic growth of the energy as a function of $n$ in this situation.

\subsection{Numerical Results}

The vortex equations of motion are not analytically solvable. We numerically solve the dimensionless equations of motion (2.46), 2.47) and (2.48) along with the accompanying boundary conditions. Below we outline the results for 


\section{ABELIAN CHERN-SIMONS VORTICES AT FINITE CHEMICAL POTENTIAL}

the quartic potential $(s=2)$ first, and subsequently summarise the results for the sextic $(s=3)$ case.

\subsubsection{Quartic potential $(s=2)$}

Negative flux solutions: A well known feature of Chern-Simons-Higgs vortices in vacuum is the ring-like profile of electric and magnetic fields [181]. The finite density vortices we have studied are qualitatively distinct and retain this feature only partially. Figure (2.2) shows the (dimensionless) scalar field $\tilde{f}(\tilde{r})$ and magnetic field $\tilde{B}(\tilde{r})$ at $\alpha=5$ and different negative values of the magnetic flux.
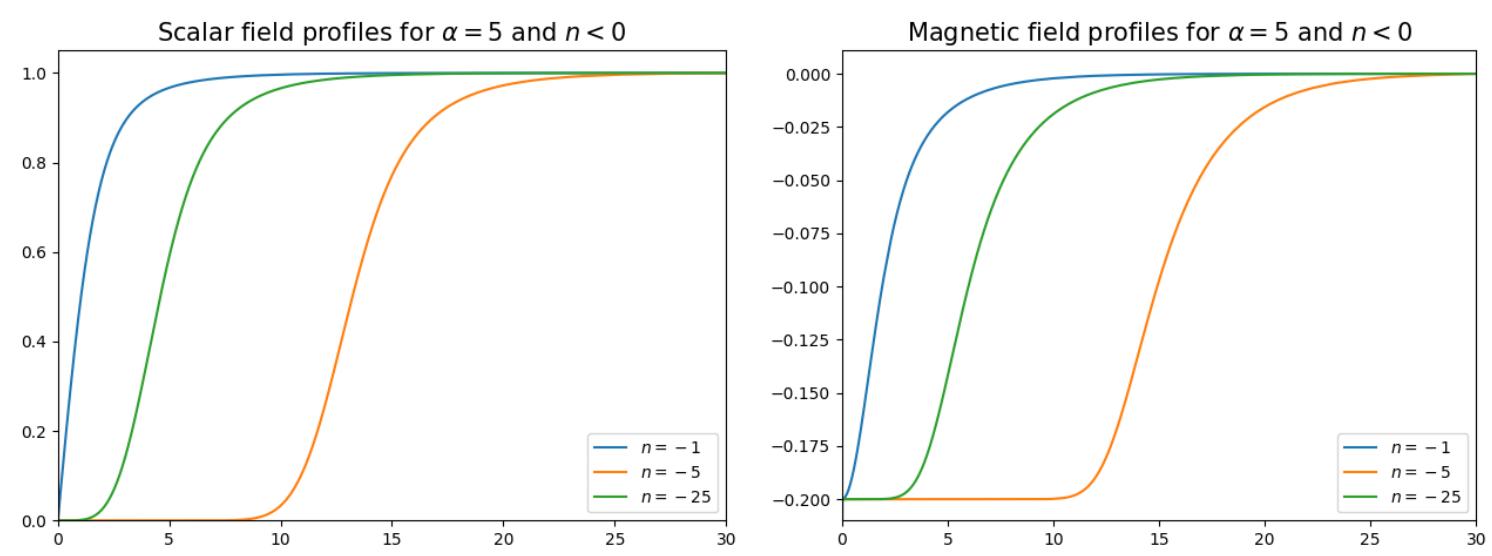

Figure 2.2: Left: The scalar field profile $\tilde{f}(\tilde{r})$ for $\alpha=5$ and negative flux. Right: The dimensionless magnetic field $\tilde{B}(\tilde{r})$ for the same values of $\alpha$ and magnetic flux.

Unlike Chern-Simons vortices in vacuum [181, 193] the magnetic field is no longer expelled from the core of the vortex. Instead, the magnetic and the scalar fields are both effectively constant inside and outside the vortex and we observe a kink-like transition in between. The value of the dimensionless magnetic field inside the vortex is $\tilde{B}(0)=-1 / \alpha=-0.2$ for $\alpha=5$. 

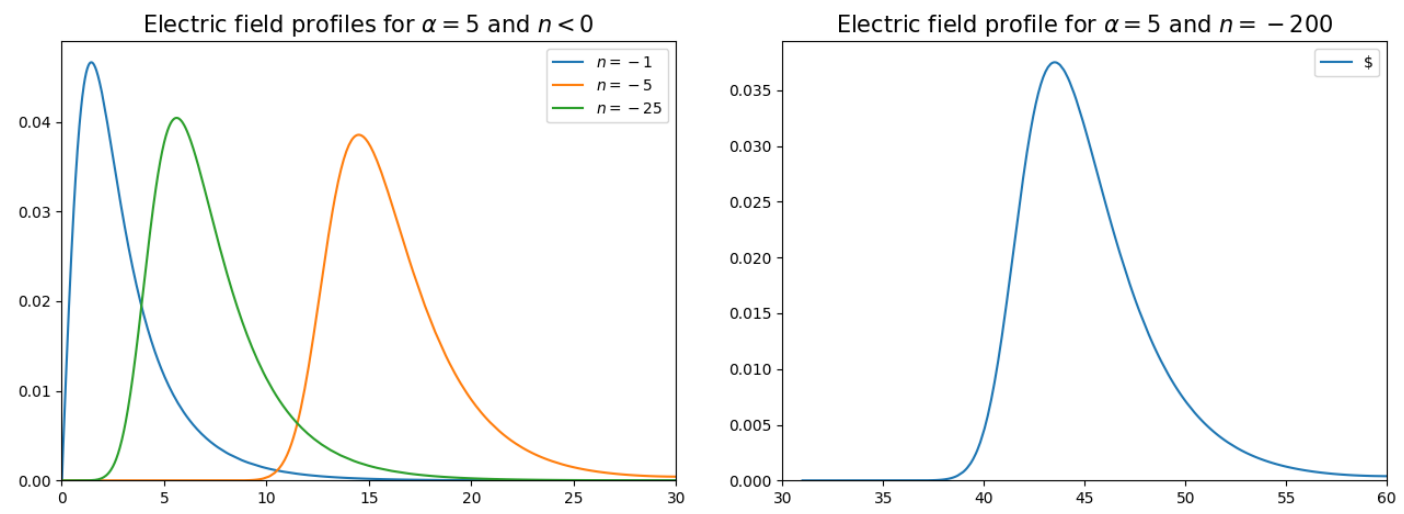

Figure 2.3: The electric field has support only at the edge of the vortex implying a ring-like profile. The width of the transition region remains fixed as $|n|$ is cranked up.

The electric field, on the other hand, has support only at the edge or the transition-region where the gradient of $A_{0}$ is significant. This is illustrated in Figure (2.3). Even for relatively low values of $|n|$, the location of the peak in the magnitude of the electric field begins to track the large $|n|$ estimate of the vortex radius in Equation 2.33:

$$
\mu R_{n}=\sqrt{2|n| \alpha}=\left\{\begin{array}{cc}
7.07 & n=-5, \alpha=5 \\
15.81 & n=-25, \alpha=5 \\
44.7 & n=-200, \alpha=5 .
\end{array}\right.
$$

The width of the ring-like transition region remains fixed as $|n|$ is increased. In particular, both the width of the ring and the peak magnitude of the electric field appear to be solely determined by $\alpha$ for large flux. 


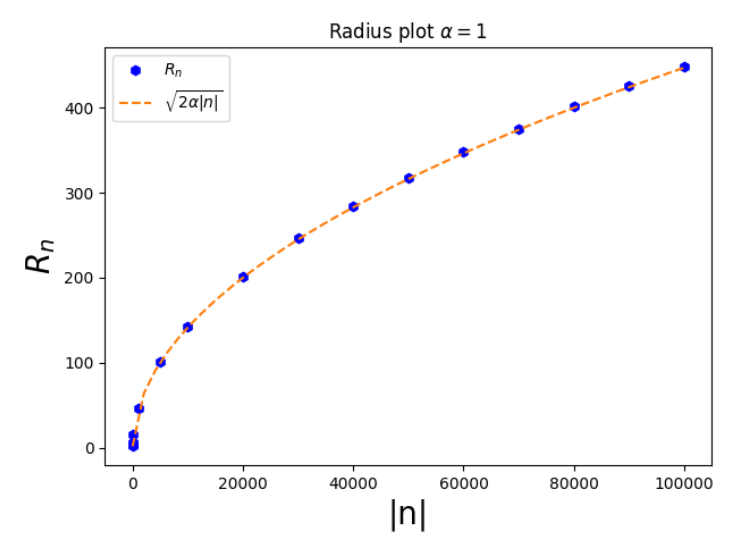

Figure 2.4: The radii of large $n$ vortex solutions follow very closely the curve $\mu R_{n}=\sqrt{2 \alpha|n|}$. We define the radius of the vortex as the position at which the dimensionless energy density falls below a threshold $\sim 10^{-4}$.

A precise agreement between the above scaling formula for the radius is obtained when the magnitude of the flux is significantly increased, as shown in Figure 2.4.

The most interesting aspect of the negative flux vortex solutions is the scaling of the energy with $|n|$. Using the energy functional (2.50), we compute the two dimensionless ratios,

$$
\frac{\mathcal{E}(n, \alpha)}{|n| \mathcal{E}(1, \alpha)} \quad \text { and } \quad \frac{\mathcal{E}(n, \alpha)}{2 \pi v^{2}|n|} \stackrel{|n| \gg 1}{\longrightarrow} \frac{\alpha}{2} .
$$

The first ratio measures the energy of the $n$-vortex relative to that of $n$ vortices each with unit (negative) flux. If this is less than unity, then the $n$-vortex has lower energy than $n$ separated (-1)-vortices, and therefore the interactions between them must be attractive (type I). Conversely, if $\mathcal{E}(n, \alpha)>|n| \mathcal{E}(1, \alpha)$, the vortex interaction is repulsive (type II). 

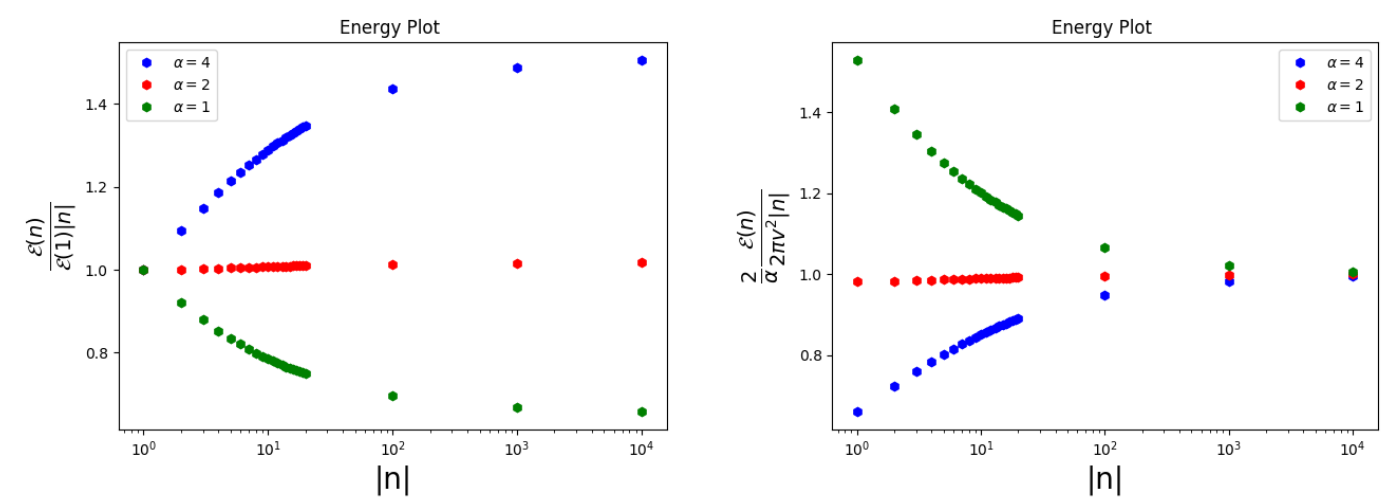

Figure 2.5: Left: This shows that $\alpha<2$ vortices are type I (attractive). They are separated from type II (repulsive) solutions for $\alpha>2$ by the $\alpha=2$ line, where the solutions have vanishing interaction energy. Right: The $\alpha$-dependence of the $n$-vortex mass formula agrees with analytical arguments at large $|n|$.

The second ratio in Equation 2.61) is the general formula for the $\alpha$-dependence of the $n$-vortex energy which was deduced from arguments for large $|n|$. Figure 2.5 shows, to significant numerical precision, that negative flux solutions with $\alpha=2$ are effectively "BPS" for any value of $|n|$, separating $\alpha>2$ solutions, which are type II (repulsive), from the solutions with $\alpha<2$, which are type I or attractive. Furthermore the $\alpha$-dependence of the energies of type I and type II vortices for large flux matches the predicted behaviour in Equation 2.61).

A surprising feature of the numerical results is how closely the energies of the vortices with $\alpha=2$ match the BPS result $2 \pi|n| v^{2}$ even for low values of $|n|$. This matching is corroborated by Figure (2.6), which shows that the vortex profile for $\alpha=2$ almost solves the first order equation $\tilde{f}^{\prime}=a \tilde{f}$. This figure also demonstrates that the vector potential inside the vortex closely follows the result for a uniform magnetic field. 

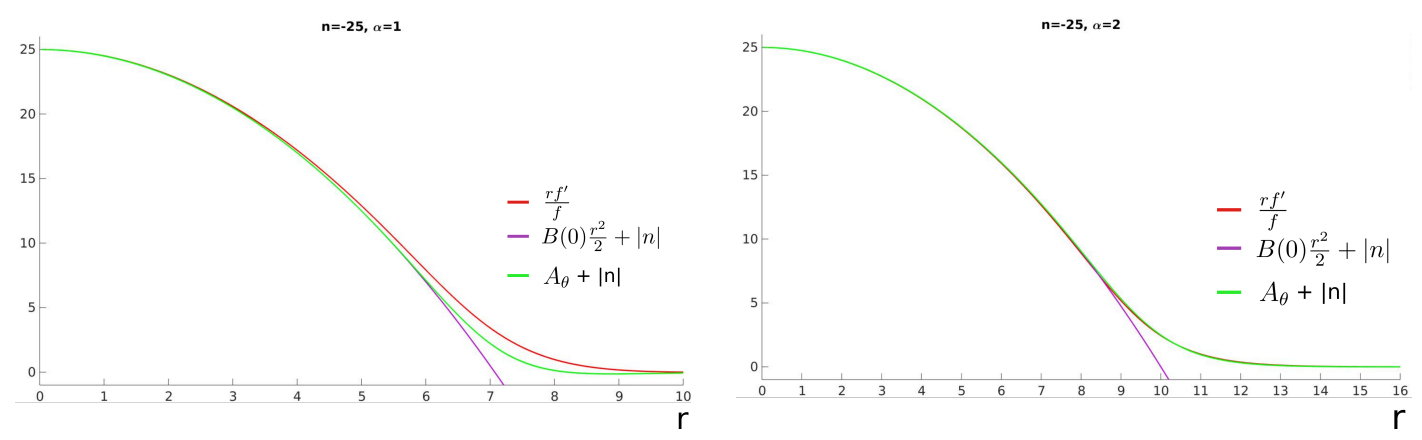

Figure 2.6: The figure shows the three quantities: $r f^{\prime} / f$ (red), $\left(A_{\theta}+|n|\right)$ (green), and $\left(-|B(0)| r^{2} / 2+|n|\right)$ (purple) evaluated on the exact numerical solutions for $\alpha=$ 1 and $\alpha=2$.

The extent of the departure of the vortex profile from an exact solution to the first order equation $\tilde{f}^{\prime}=a f$ is shown in Figure (2.7). Evaluated on the $\alpha=2$ solution, the quantity $\left(\tilde{f}^{\prime}-a \tilde{f}\right)$ deviates minimally from zero near the edge of the vortex.
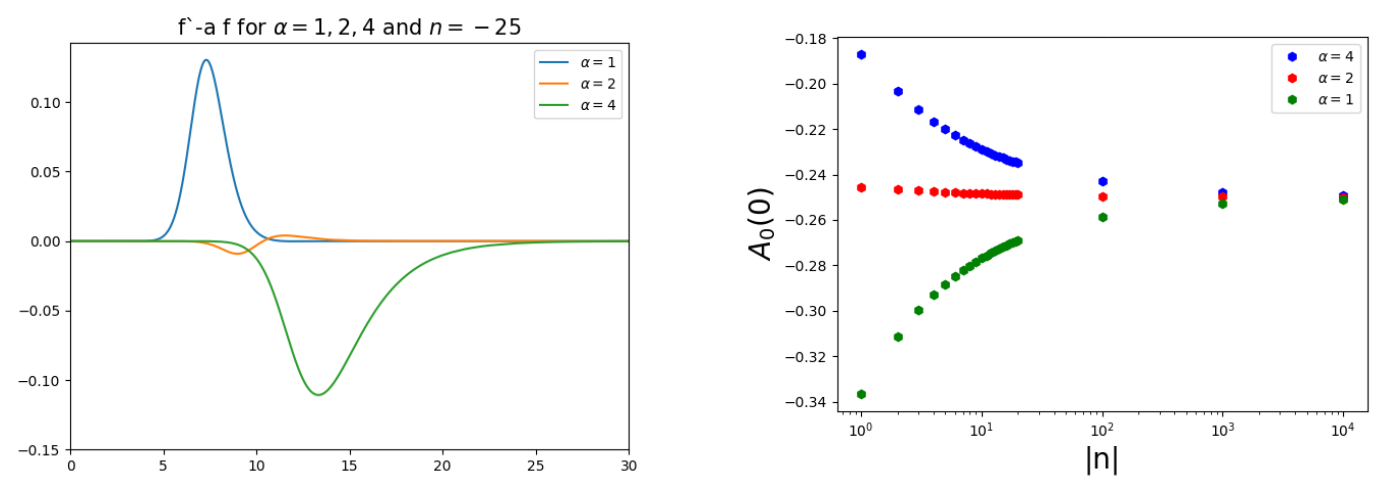

Figure 2.7: Left: $\left(\tilde{f}^{\prime}-a \tilde{f}\right)$ plotted for 3 values of $\alpha$ including the critical case $\alpha=2$. Right: The value of $\tilde{A}_{0}$ at the origin for different $\alpha$ as a function of $|n|$.

Yet another measure of the relevance of the first order equation $\tilde{f}^{\prime}=a \tilde{f}$ for the $\alpha=2$ solutions is given by the value of $\tilde{A}_{0}(0)$. The value of the electrostatic potential at the origin is not fixed as a boundary condition, but an output of the solution. Let us use the equation of motion for the electric field 2.47 in 
conjunction with the first order equation at $\alpha=2$,

$$
\tilde{A}_{0}^{\prime}=\frac{1}{2} a \tilde{f}^{2} \quad \stackrel{\tilde{f}^{\prime}=a \tilde{f}}{\longrightarrow} \quad \tilde{A}_{0}=\frac{1}{4}\left(\tilde{f}^{2}-1\right),
$$

where the integration constant on the right hand side is fixed by requiring that $\tilde{A}_{0}$ vanishes as $r \rightarrow \infty$. We therefore arrive at a prediction,

$$
\left.\tilde{A}_{0}(0)\right|_{\alpha=2}=-\frac{1}{4}
$$

This is precisely what we see in Figure (2.7) for the $\alpha=2$ solution. However, we also find the unexpected feature that $\tilde{A}_{0}(0)$, for other values of $\alpha$, approaches $-1 / 4$ at large $|n|$. This suggests that solutions with generic $\alpha$ and large $|n|$ are not approximate solutions to the first order equation ${ }^{1}$.

Positive flux solutions: We have explained how positive flux vortex solutions are qualitatively distinct from negative $n$ solutions. In certain limits (large $\alpha$ ) they closely resemble Chern-Simons vortex solutions in vacuum. The majority of the flux resides in the ring region or edge of the solution as $n$ is increased (see Figure (2.8).
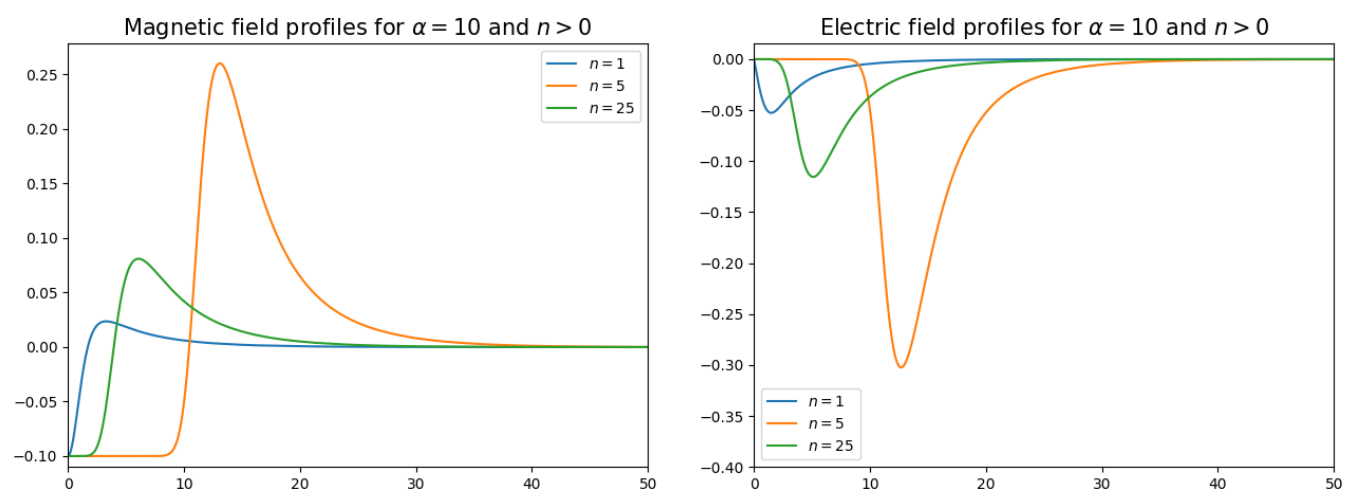

Figure 2.8: Magnetic and electric fields for positive flux vortices have support near the edge of the vortex and their peak values grow without bound as $n$ is increased.

\footnotetext{
${ }^{1}$ For generic $\alpha$, Equation 2.47 along with the first order equation $\tilde{f}^{\prime}=a \tilde{f}$, would imply $\tilde{A}_{0}(0)=-\frac{1}{2 \alpha}$.
} 


\section{ABELIAN CHERN-SIMONS VORTICES AT FINITE CHEMICAL POTENTIAL}

In Figure 2.9) we have depicted a comparison between magnetic field profiles of the pure Chern-Simons vortex (also known as the Jackiw-Lee-WeinbergHong-Kim-Pac (JLW-HKP) vortex [63, 64, 199]) and the finite density vortex studied in this section, in order to highlight the similarity in the $\mu \rightarrow 0$ limit. This ring-like profile in both cases is in contrast to the abelian Higgs model's disk-like structure, which we observed in Figure (1.3) earlier. The transition between these two types of behaviours (ring-like in CS and disk-like in abelian Higgs) has been more extensively studied by Bolognesi $\mathcal{E}$ Gudnason [193].

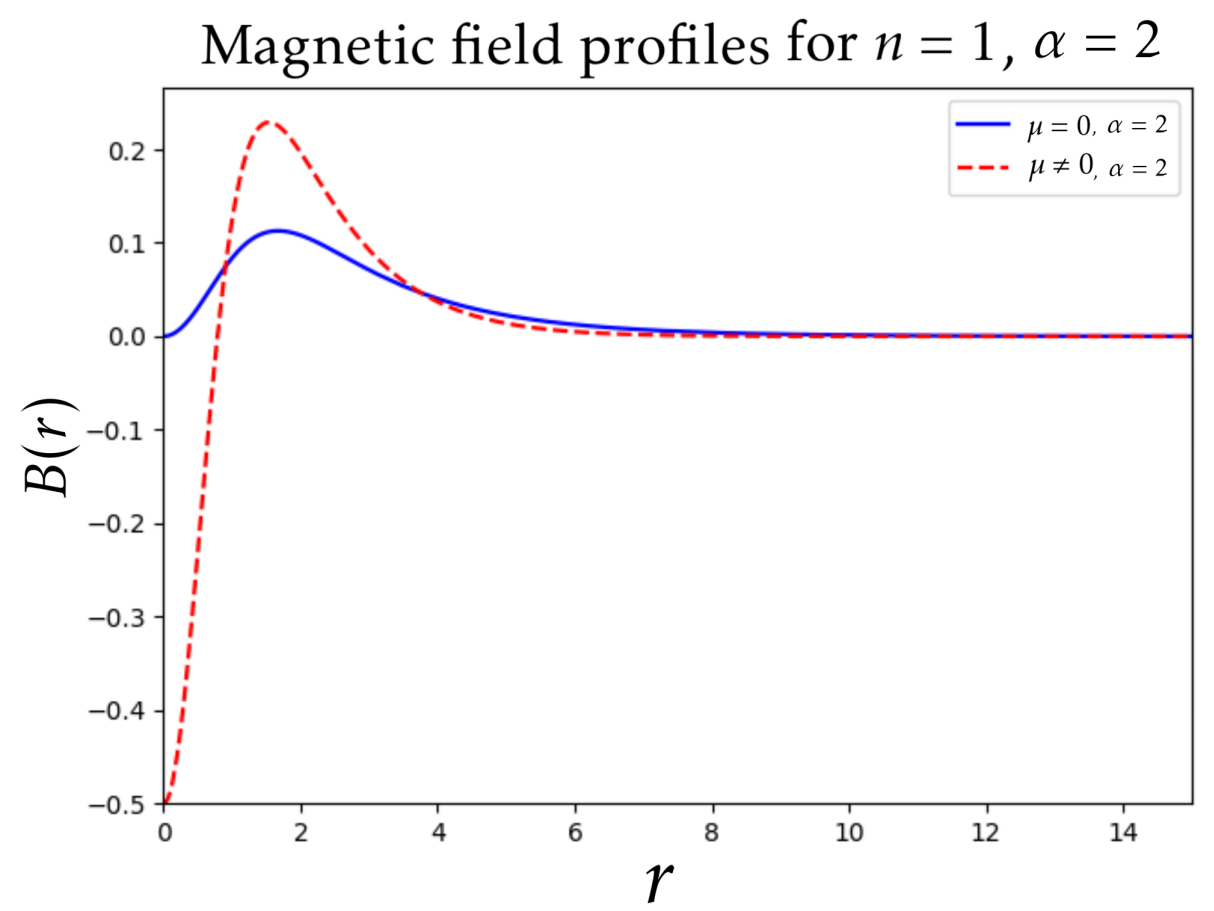

Figure 2.9: Magnetic field profiles comparison for $\mu=0$ and $\mu \neq 0$, in the case of $n=1$. Here the red line shows the $\mu \neq 0$ vortex, while the blue line shows the $\mu=0$ case. Strictly speaking, a vortex would not exist in such a limit, since the symmetry breaking is driven by the chemical potential. However, here we have depicted the vortex in a theory with a negative mass squared $\left(\mathrm{m}^{2}\right)$ term in the potential. Since we have excluded 6 th order terms (unlike the original studies of JLW-HKP) we may similarly describe the solutions using only one parameter. Due to its similarity with the $m=0, \mu \neq 0$ case, we have chosen to denote this parameter as $\alpha=\frac{k m}{4 \pi v^{2}}$, to emphasise the analogy. 
In Figure 2.10$)$ the dependence of the $n$-vortex energy on $|n|$, is displayed for the negative and positive flux solutions, and as expected, the latter are more massive. It is surprising that $\mathcal{E}(n) /|n|$ appears to grow faster than $|n|$.

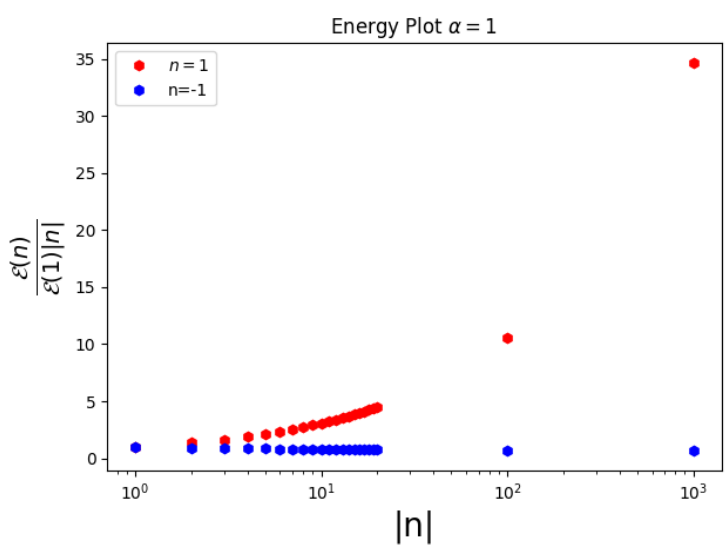

Figure 2.10: This figure shows the energy of a vortex per unit of a flux, measured in units of a flux 1 vortex. Positive flux vortices have higher energy than their negative flux counterparts.

\subsubsection{Sextic potential $(s=3)$}

Finally we turn to numerical results for the higher power law potentials, in particular the $s=3$ or sextic potential. We do not expect to see major qualitative differences from the $s=2$ case. One special feature of the quartic potential $(s=2)$ is that when $\alpha=2$, there is a precise cancellation of the scalar potential energy contribution to the energy functional. This is not the case for general power laws. Nevertheless there is an approximate cancellation when evaluated on the vortex background at the critical value of $\alpha=\frac{s}{s-1}$. The critical value for the sextic potential is $\alpha=\frac{3}{2}$. The profiles for the negative flux vortex with sextic and quartic potentials are shown on the same plot in Figure 2.11). For the same values of the dimensionless parameter $\alpha$ there is very little difference between the two systems. The transition between the two phases is slightly steeper for the sextic potential. The ratios of the energies of the $n$-vortex to single vortex and the BPS value $\left(2 \pi v^{2} n\right)$ are shown in Figure 2.12. 


\section{ABELIAN CHERN-SIMONS VORTICES AT FINITE CHEMICAL} POTENTIAL
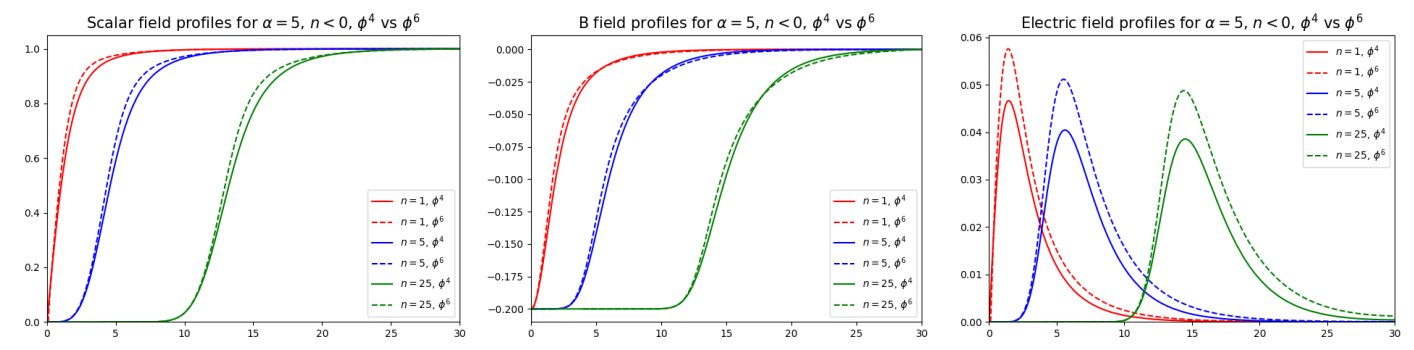

Figure 2.11: Vortex profiles with negative winding number for quartic(solid) and sextic(dashed) potentials. The figure shows the scalar, magnetic and electric fields profiles, respectively.
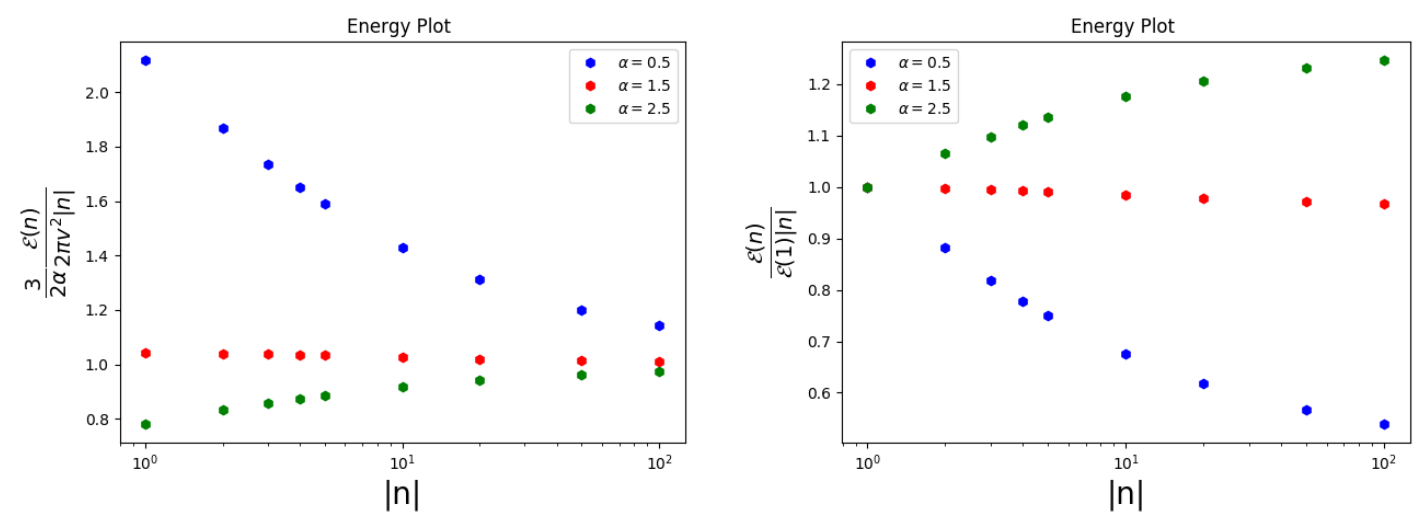

Figure 2.12: The scaling of the energy of the $n$-vortex with $|n|$ and $\alpha$, with sextic potential. The plots show two different normalisations. On the left we have normalised the energy with respect to the value of a BPS vortex, whereas on the right we have used the single flux vortex with its respective value of $\alpha$ as normalisation.

The dependence of the energy on $|n|$ and $\alpha$ matches the prediction from Equation (2.58), and we find a transition line between type I and type II vortices at the critical coupling $\alpha_{c}=\frac{3}{2}$ which represents the marginally bound "BPS" case for the sextic potential. Again the value of $\tilde{A}_{0}(0)$ indicates that the profiles almost satisfy the first order equation which would imply,

$$
\tilde{A}_{0}(0)=-\frac{1}{2 \alpha_{c}}=-\frac{1}{3}
$$

This is indeed what we observe in Figure (2.13). All large $n$ vortex profiles 
approach this value.

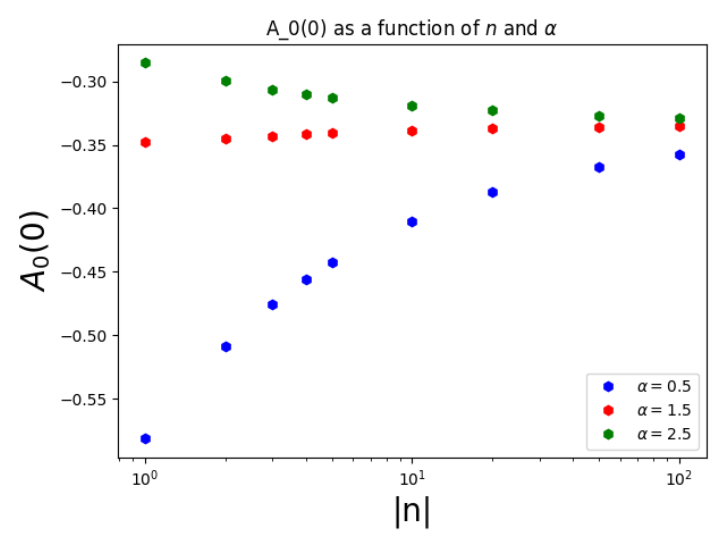

Figure 2.13: Value of $\tilde{A}_{0}$ at the origin for different values of $\alpha$, as a function of $|n|$, with the sextic potential.

\subsection{Discussion}

We have studied abelian Chern-Simons vortices in the presence of a chemical potential driving the theory into the Higgs phase. The numerical solutions reveal several features which are in line with the physical picture presented in an analytically solvable (non-relativistic) supersymmetric model [190, 200]. The configurations with large (negative) flux show precise BPS-like scaling of energy/grand potential and appear (numerically) to closely solve first order equations. It would be interesting to make use of the large flux limit to understand the edge excitations of the vortex droplet. We expect that some of the lessons learnt from analysing this system will be of use in $S U(N)$ and $U(N)$ Chern-Simons-scalar theories with a particle number chemical potential. In these theories the ground state putatively breaks rotational invariance due to condensation of vector fields [182], and depending on whether we are in the $S U(N)$ or $U(N)$ theory, particle number is ungauged or gauged, and we will have superfluid or superconducting vortices. 
2. ABELIAN CHERN-SIMONS VORTICES AT FINITE CHEMICAL POTENTIAL 


\section{Chapter 3}

\section{Roton-Phonon Excitations in Chern-Simons Matter Theory at Finite Density}

\subsection{Introduction}

Relativistic field theories in three dimensions consisting of Chern-Simons gauge fields coupled to matter have been conjectured to enjoy a Bose-Fermi/levelrank duality symmetry [39]. Mounting evidence for the conjecture has appeared in various forms. These include detailed aspects of correlators [39, 42, 105, 201, 202] and S-matrices [203, 204] in large $N$ vector models coupled to Chern-Simons gauge fields in the 't Hooft limit when the theory becomes exactly solvable. Further, the large $N$ thermal partition functions have been shown to exhibit Bose-Fermi duality as the 't Hooft coupling is varied [39, 43, 81, 187, 188]. A crucial role in this is played by the non-trivial eigenvalue distributions of the holonomy matrix around the Euclidean thermal circle, and the duality manifests itself in various phases characterised by the large $N$ eigenvalue distributions. The finite $N$ versions of the duality can be precisely formulated [40], and include an intricate web of abelian dualities [74, 185, 186] with particle-vortex duality as one of its strands.

In this work, motivated by the goal of understanding the manifestations of Bose-Fermi duality at finite density, we study the zero temperature ground 


\section{ROTON-PHONON EXCITATIONS IN CHERN-SIMONS MATTER THEORY AT FINITE DENSITY}

states of a fundamental scalar coupled to Chern-Simons gauge fields in the presence of a chemical potential for particle number. In particular, we will be mainly interested in finite density ground states in the (semi-)classical limit which spontaneously break the global $U(1)_{B}$ particle number symmetry. This is a subtle issue in $2+1$ dimensions, as any finite temperature will result in thermal fluctuations that, by the Coleman-Mermin-Wagner theorem [205, 206], can destroy long-range order. For this reason, in this thesis we limit ourselves to the system at zero temperature. At non-zero temperature and finite density in the absence of condensates, exact results at large $N$ for Chern-Simons theory with a fundamental fermion [189, 207] show non-trivial agreement at strong 't Hooft coupling 11 with its weakly interacting bosonic counterpart.

In our analysis of the Chern-Simons-scalar system we assume a classical limit, i.e. the Chern-Simons level $k$ is large (but finite), and any other scalar self-couplings taken to be suitably weak so that the semi-classical description applies. Our main findings are summarised below:

- We find that the theory with $S U(N)$ gauge group, Chern-Simons level $k$ and non-zero chemical potential for particle number, exhibits a zero temperature ground state where the scalar field condenses and all gauge fields acquire non-commuting background expectation values. This ground state breaks the $S U(N)$ gauge symmetry completely and spontaneously breaks the global $U(1)_{B}$ particle number symmetry. While spatial rotations act non-trivially on the background gauge potentials, they can be undone by a $U(1)_{C}$ subgroup of global $S U(N)$ transformations. Thus gauge invariant operators acquire rotationally invariant expectation values. The scalar vacuum expectation value (VEV) itself is left invariant by a combination of the flavour $U(1)_{B}$ and global colour $U(1)_{C}$ rotations.

- For the $S U(2)$ theory, assuming $k \gg 1$, we obtain the spectrum of physical fluctuations and their dispersion relations in the Bose condensed ground state. The fluctuation spectrum exhibits a massless phonon mode with

\footnotetext{
${ }^{1}$ The 't Hooft coupling $\lambda$ is defined in the limit $N, k \rightarrow \infty$ ( $k$ is the Chern-Simons level) where $\lambda \equiv \frac{N}{k}$, ranging between 0 and 1 .
} 
linear dispersion relation for the frequency $\omega \sim c_{s}|\mathbf{k}|$, for low spatial momenta $\mathbf{k}$, accompanied by a local maximum and a roton minimum at some finite spatial momentum. Roton-maxon excitations are well known within the context of superfluidity in ${ }^{4} \mathrm{He}$ [208, 209] and explain various physical characteristics such as heat capacity and the superfluid critical velocity. The roton minimum, for instance, lowers the superfluid critical velocity to below the speed of sound, as can be understood by applying the Landau criterion [208, 209]. In the context of this chapter, we understand the appearance of the roton minimum as a consequence of level crossing of states. In the strict limit $k \rightarrow \infty$ when the Chern-Simons fields decouple, the interacting scalar theory has a Bose condensate with two gapless excitations at zero momentum, one with quadratic and the other with a linear dispersion relation. At large but finite $k$, the former acquires a gap at zero momentum, and the putative intersection between the linear and quadratic dispersion curves is replaced by a roton-maxon pair in the diagonalised spectrum. The background VEVs for the gauge fields are directly responsible for these features. Roton-like excitations with very similar origin, i.e. constant background gauge fields, have been identified in Yang-Mills-Higgs systems at finite density in 3+1 dimensions [183].

We find that the roton minimum in the phonon dispersion relation persists in the free scalar theory coupled to Chern-Simons gauge fields (at large $k$ ). In this case the only dimensionful scale is provided by the chemical potential which can be rescaled to unity and the resulting spectra and dispersion relations acquire a universal form.

- For the general $S U(N)$ case an interesting picture emerges. The $N \times N$ matrices of VEVs for the Chern-Simons gauge fields provide finite dimensional versions of harmonic oscillator creation and annihilation operators. In particular, they can be viewed as the non-commuting coordinates of $N$ particles in a disc of fixed radius. The same matrices have been used to describe the ground state of the quantum Hall droplet [92, 93]. Fluctuations about the finite density ground state may thus be viewed 


\section{ROTON-PHONON EXCITATIONS IN CHERN-SIMONS MATTER THEORY AT FINITE DENSITY}

as fluctuations of this droplet (in configuration space), carrying spatial momentum and frequency.

The zero temperature finite density properties of the Chern-Simons-scalar system present a range of physical phenomena interesting in their own right. Importantly, they provide predictions for the fermionic dual. The $S U(N)_{k}$ theory with a fundamental scalar (and level $k$ ) is level-rank dual to the $U(k-N)_{-k,-N}$ theory ${ }^{1}$ with a fundamental fermion [40]. In particular, the free scalar coupled to Chern-Simons fields is dual to the Chern-Simons plus critical fermion theory [210]. It is clearly of great interest to understand whether features of the spectrum of the weakly coupled scalar system can be understood from the conjectured fermionic dual at strong coupling.

This chapter is organised as follows. In Section (3.2) we study the Bose condensed ground state of the $S U(2)$ system in the classical limit. In Section 3.3 we find the spectrum of quadratic fluctuations after gauge fixing, and identify the phonon-roton branch for different regimes of parameters. Section (3.4) is devoted to the generalisation of the classical vacuum structure to general $N>2$. Finally we outline a number of questions for future study in Section (3.7).

\subsection{The $S U(2)_{k}$ Theory}

We consider Chern-Simons theory with $S U(2)$ gauge group and one scalar flavour transforming in the fundamental representation. Working with an anti-hermitean gauge potential $A_{\mu}$,

$$
A_{\mu}=A_{\mu}^{(a)} t^{a}, \quad t^{a} \equiv \frac{i}{2} \sigma^{a}, \quad a=1,2,3,
$$

\footnotetext{
${ }^{1}$ The two subscripts denote the Chern-Simons levels of the $S U(k-N)$ and $U(1)$ factors of the gauge group respectively.
} 
where $\left\{\sigma^{a}\right\}$ are the Pauli matrices and $\left\{A_{\mu}^{(a)}\right\}$ are real valued fields, the ChernSimons action with (quantised) level $k$ is then,

$$
S_{C S}=\frac{k}{4 \pi} \int d^{3} x \epsilon^{\mu \nu \rho} \operatorname{Tr}\left(A_{\mu} \partial_{\nu} A_{\rho}+\frac{2}{3} A_{\mu} A_{v} A_{\rho}\right)
$$

This is the action for both Euclidean $(+++)$ and Lorentzian $(-++)$ signatures. The Wick rotation from Lorentzian to Euclidean space-time is implemented by the replacement $t \rightarrow-i \tau$ and $A_{0} \rightarrow i A_{0}$, which together leave $S_{C S}$ invariant. In Lorentzian signature, the complete action involving Chern-Simons and matter fields has the general form,

$$
S=S_{\text {matter }}+S_{C S}
$$

where, in Lorentzian signature $(-++)$, for a scalar $\Phi$ transforming in the fundamental representation of $S U(2)$,

$$
\begin{aligned}
& S_{\text {matter }}=-\int d^{3} x\left(\left(D_{\mu} \Phi\right)^{\dagger}\left(D^{\mu} \Phi\right)+V\left(\Phi^{\dagger} \Phi\right)\right), \\
& D_{\mu} \equiv \partial_{\mu}+A_{\mu} .
\end{aligned}
$$

The theory possesses a global $U(1)$ symmetry which we refer to as "baryon number" or $U(1)_{B}$,

$$
U(1)_{B}: \quad \Phi \rightarrow e^{i \vartheta} \Phi,
$$

generated by a phase rotation of $\Phi$. The corresponding conserved current is

$$
j_{B}^{\mu}=i\left[\left(D^{\mu} \Phi\right)^{\dagger} \Phi-\Phi^{\dagger} D^{\mu} \Phi\right]
$$

The chemical potential $\mu_{B}$ is a Lagrange multiplier for the $U(1)_{B}$ charge. In Lorentzian signature, it therefore appears in the Lagrangian as a time component for a background $U(1)_{B}$ gauge field:

$$
D_{v} \rightarrow D_{v}+i \mu_{B} \delta_{v, 0}
$$




\section{ROTON-PHONON EXCITATIONS IN CHERN-SIMONS MATTER THEORY AT FINITE DENSITY}

\subsubsection{Classical ground states with $\mu_{B} \neq 0$}

The coupling of the Chern-Simons fields to the matter sector is controlled by $1 / \sqrt{k}^{1}$. In the limit $k \rightarrow \infty$, the scalar field $\Phi$ with $\mu_{B} \neq 0$ has the potential:

$$
V_{\text {scalar }}\left(\mu_{B}, k \rightarrow \infty\right)=V\left(\Phi^{\dagger} \Phi\right)-\mu_{B}^{2} \Phi^{\dagger} \Phi
$$

As usual, the effective negative mass squared due to the chemical potential drives the system to form a Bose condensate for large enough $\mu_{B}$. The tree level three dimensional scalar potential (at $\mu_{B}=0$ ) can be taken to be of the form,

$$
V\left(\Phi^{\dagger} \Phi\right)=m^{2} \Phi^{\dagger} \Phi+g_{4}\left(\Phi^{\dagger} \Phi\right)^{2}+g_{6}\left(\Phi^{\dagger} \Phi\right)^{3},
$$

where we have allowed for relevant and marginal operators in the scalar potential. Assuming that the ground state of the theory with $\mu_{B} \neq 0$ is static and translation invariant, we look for vacuum solutions with all terms involving derivatives being set to zero. Anticipating a scalar condensate at the classical level ${ }^{2}$, we can always choose gauge rotations to take the VEV to be real and of the form,

$$
\langle\Phi\rangle=\left(\begin{array}{l}
0 \\
v
\end{array}\right) \quad v \in \mathbb{R}
$$

We then collectively view all non-derivative terms as potential energy contributions:

$$
\begin{aligned}
V_{C S}+V_{\text {scalar }} & =-\frac{k}{4 \pi} \epsilon^{\mu \nu \rho} A_{\mu}^{(1)} A_{\nu}^{(2)} A_{\rho}^{(3)}-\frac{v^{2}}{4}\left[\left(A_{0}^{(1)}\right)^{2}+\left(A_{0}^{(2)}\right)^{2}+\left(A_{0}^{(3)}-2 \mu_{B}\right)^{2}\right] \\
& +\frac{v^{2}}{4} \sum_{i=1,2}\left[\left(A_{i}^{(1)}\right)^{2}+\left(A_{i}^{(2)}\right)^{2}+\left(A_{i}^{(3)}\right)^{2}\right]+m^{2} v^{2}+g_{4} v^{4}+g_{6} v^{6} .
\end{aligned}
$$

One consistent extremum is given by $v=0$, and all gauge fields also vanishing. This is the trivial solution. However, this solution cannot dominate the grand

\footnotetext{
${ }^{1}$ This can be understood via the rescaling $A_{\mu} \rightarrow A_{\mu} / \sqrt{k}$, following which the Chern-Simons action is order 1 in the large $k$ limit.

${ }^{2}$ The analysis will remain purely classical and at zero temperature at this stage. At finite temperature, we know that quantum thermal fluctuations in $2+1$ dimensions preclude symmetry breaking of continuous global symmetries.
} 
canonical ensemble for generic values of the chemical potential. In particular, the scalar field theory without Chern-Simons terms $\left(k^{-1} \rightarrow 0\right)$, and at weak coupling $\left(g_{4} \ll m, g_{6} \ll 1\right)$, develops a Bose condensate when $\left|\mu_{B}\right|>m$. This non-trivial phase with $v \neq 0$ must persist when the coupling to Chern-Simons gauge fields is turned on. In order to arrive at a static and translationally invariant ground state, we need to find the minima of the potential energy function (3.11). We adopt a notation which is appropriate for $S U(2)$ by introducing three-vectors in the internal "isospin" directions:

$$
\mathbf{A}_{\mu} \equiv\left(\left\langle A_{\mu}^{(1)}\right\rangle,\left\langle A_{\mu}^{(2)}\right\rangle,\left\langle A_{\mu}^{(3)}\right\rangle\right)^{T}, \quad \mathbf{e}^{a} \equiv\left(\delta^{a, 1}, \delta^{a, 2}, \delta^{a, 3}\right)^{T}
$$

In terms of these, the vacuum equations determining the ground state are (here the ' $x$ ' and ' ' symbols denote cross- and dot-products in the internal space):

$$
\begin{aligned}
& v^{2} \mathbf{A}_{y}=\frac{k}{2 \pi} \mathbf{A}_{0} \times \mathbf{A}_{x}, \quad v^{2} \mathbf{A}_{x}=\frac{k}{2 \pi} \mathbf{A}_{y} \times \mathbf{A}_{0}, \\
& -v^{2}\left(\mathbf{A}_{0}-2 \mu_{B} \mathbf{e}^{3}\right)=\frac{k}{2 \pi} \mathbf{A}_{x} \times \mathbf{A}_{y}, \\
& \frac{v}{2}\left[\left(\mathbf{A}_{0}-2 \mu_{B} \mathbf{e}^{3}\right)^{2}-\left(\mathbf{A}_{x}\right)^{2}-\left(\mathbf{A}_{y}\right)^{2}\right]=\frac{\partial V}{\partial v} .
\end{aligned}
$$

The two equations in 3.13 together imply that $\mathbf{A}_{0}, \mathbf{A}_{x}$ and $\mathbf{A}_{y}$ are mutually orthogonal in the internal isospin directions, and that

$$
\left|\mathbf{A}_{x}\right|=\left|\mathbf{A}_{y}\right|, \quad\left|\mathbf{A}_{0}\right|=\frac{2 \pi v^{2}}{|k|}, \quad \operatorname{sgn}\left[\left(\mathbf{A}_{x} \times \mathbf{A}_{y}\right) \cdot \mathbf{A}_{0}\right]=\operatorname{sgn}(k) .
$$

Next, by taking the cross-product of Equation 3.14 with $\mathbf{A}_{0}$, we deduce that $\mathbf{A}_{0}=\left\langle A_{0}^{(3)}\right\rangle \mathbf{e}^{3}$ :

$$
\begin{aligned}
\left(\mathbf{A}_{x} \times \mathbf{A}_{y}\right) \times \mathbf{A}_{0} & =\mathbf{A}_{y}\left(\mathbf{A}_{0} \cdot \mathbf{A}_{x}\right)-\mathbf{A}_{x}\left(\mathbf{A}_{0} \cdot \mathbf{A}_{y}\right) \\
& =\frac{k}{2 \pi v^{2}}(\mathbf{A}_{y} \underbrace{\left(\mathbf{A}_{0} \cdot\left(\mathbf{A}_{y} \times \mathbf{A}_{0}\right)\right)}_{=0}-\mathbf{A}_{x} \underbrace{\left(\mathbf{A}_{0} \cdot\left(\mathbf{A}_{0} \times \mathbf{A}_{x}\right)\right)}_{=0})=0 \\
& \Longrightarrow \mathbf{A}_{0} \times \mathbf{e}^{3}=0
\end{aligned}
$$




\section{ROTON-PHONON EXCITATIONS IN CHERN-SIMONS MATTER THEORY AT FINITE DENSITY}

where we have used Equation (3.13) in the second line. Finally, combining Equations (3.14) and (3.15), we obtain conditions on the magnitudes of the background field expectation values:

$$
\begin{aligned}
& \left|\mathbf{A}_{x}\right|^{2}=\left|\mathbf{A}_{y}\right|^{2}=\frac{2 \pi v^{2}}{|k|}\left|\left\langle A_{0}^{(3)}\right\rangle-2 \mu_{B}\right|, \\
& \left(\left\langle A_{0}^{(3)}\right\rangle-2 \mu_{B}\right)^{2}-\frac{4 \pi v^{2}}{|k|}\left|\left\langle A_{0}^{(3)}\right\rangle-2 \mu_{B}\right|-\frac{2}{v} \frac{\partial V}{\partial v}=0 .
\end{aligned}
$$

To proceed further, it is useful to work with the (isospin) basis elements

$$
\mathbf{A}_{0}=\eta \frac{2 \pi v^{2}}{|k|} \mathbf{e}^{3}, \quad \mathbf{A}_{x}=a_{1} \mathbf{e}^{1}+a_{2} \mathbf{e}^{2}, \quad \mathbf{A}_{y}=\eta \operatorname{sgn}(k)\left(-a_{2} \mathbf{e}^{1}+a_{1} \mathbf{e}^{2}\right),
$$

where $\eta= \pm 1$ and $a_{1,2} \in \mathbb{R}$. Using the equations of motion (3.13) and (3.14) we then find that

$$
\eta=\operatorname{sgn}\left(\mu_{B}\right), \quad\left(a_{1}\right)^{2}+\left(a_{2}\right)^{2}=\frac{4 \pi v^{2}}{|k|}\left(\left|\mu_{B}\right|-\frac{v^{2} \pi}{|k|}\right), \quad\left|\mu_{B}\right|>\frac{\pi v^{2}}{|k|} .
$$

The classical configuration is endowed with a non-zero $U(1)_{B}$ charge density,

$$
\left\langle j_{B}^{0}\right\rangle=\operatorname{sgn}\left(\mu_{B}\right) \frac{2 \pi v^{4}}{|k|},
$$

with vanishing $U(1)_{B}$ currents. To calculate the scalar VEV we need the form of the tree level potential. For simplicity we set $g_{6}=0$. With a quartic potential there exists a unique solution ${ }^{1}$ for the vacuum expectation value (3.19),

$$
v^{2}=\frac{|k|}{3 \pi}\left(\frac{g_{4}|k|}{\pi}+2\left|\mu_{B}\right|-\sqrt{\left(\frac{g_{4}|k|}{\pi}+2\left|\mu_{B}\right|\right)^{2}-3\left(\mu_{B}^{2}-m^{2}\right)}\right),
$$

which also satisfies the condition (3.20). As expected, the VEV is real only when $\mu_{B}^{2}>m^{2}$. Further, in the large $k$ limit, when the Chern-Simons gauge fields decouple, the value of the VEV approaches $v^{2} \simeq\left(\mu_{B}^{2}-m^{2}\right) / 2 g_{4}$. This is, of course, the scalar VEV in the pure scalar theory in the Bose condensed phase.

\footnotetext{
${ }^{1}$ The second root for $v^{2}$ yields $v^{2}>\left|\mu_{B} k\right| / 2 \pi$ and violates the condition in Equation 3.20.
} 
In the massless theory, the scalar VEV is controlled by the dimensionless combination $\left|\pi \mu_{B} / g_{4} k\right|$ :

$$
\begin{aligned}
m=0: \quad v^{2} & =\frac{\left|\mu_{B} k\right|}{2 \pi} f(\tilde{\mu}), \quad \tilde{\mu} \equiv \frac{\pi\left|\mu_{B}\right|}{g_{4}|k|}, \\
f(\tilde{\mu}) & =\frac{2}{3}\left(\tilde{\mu}^{-1}+2-\sqrt{\left(\tilde{\mu}^{-1}+2\right)^{2}-3}\right),
\end{aligned}
$$

where $f(\tilde{\mu})$ is monotonically increasing with $f(0)=0$ and $f(\infty) \simeq \frac{2}{3}$. A noteworthy point here is that the scalar VEV exists even when $g_{4}$ technically vanishes. More generally, one may view the semi-classical limit in which the condensate is well defined as $\left(g_{4} / \mu_{B}\right) \rightarrow 0$ and $k \rightarrow \infty$ such that $g_{4} k / \mu_{B}$ is kept fixed.

Free energy: For static configurations we can compute the free energy density by evaluating the potential energy function on the ground state. In terms of the VEV, the free energy is,

$$
F=v^{2}\left[g_{4} v^{2}+m^{2}-\left(\left|\mu_{B}\right|-\frac{\pi v^{2}}{k}\right)^{2}\right] .
$$

It is easy to check that (assuming $\left|\mu_{B}\right|>m$ ) the function is negative definite. In the massless case, the free energy of the Bose condensed phase is determined by the function $f(\tilde{\mu})$ :

$$
\left.F\right|_{m=0}=\frac{\left|\mu_{B}^{3} k\right|}{4 \pi} \frac{f(\tilde{\mu})}{\tilde{\mu}}\left[f(\tilde{\mu})-\frac{\tilde{\mu}}{2}(f(\tilde{\mu})-2)^{2}\right],
$$

which is a negative definite, monotonically decreasing function of $\tilde{\mu}$. Therefore, in the semi-classical regime, the non-trivial vacuum dominates over the trivial one which displays vanishing VEVs for all fields. For instance, in the massless theory with $g_{4}=0$, the free energy in the Higgsed phase is

$$
\left.F\right|_{m=0, g_{4}=0}=-4 \frac{\left|\mu_{B}^{3} k\right|}{27 \pi},
$$




\section{ROTON-PHONON EXCITATIONS IN CHERN-SIMONS MATTER THEORY AT FINITE DENSITY}

valid in the semi-classical limit $k \gg 1$. Quantum corrections are parametrically suppressed in this limit. In this case the theory enters the Higgsed phase for any non-zero chemical potential, while the theory with vanishing chemical potential is conformal. When the mass is non-zero and the chemical potential is dialed past the classical threshold value $\mu_{B}=m$, following a second order phase transition, the theory enters a Bose condensed Higgs phase. The symmetric phase is unstable beyond this point. This interpretation is supported by the plot (Figure (3.1)) of the free energy as a function of the VEV $v$ (taking $g_{4}=0$ for simplicity).

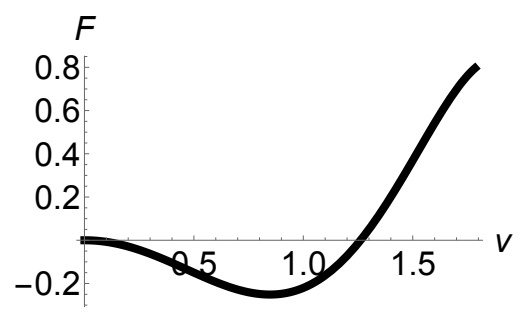

Figure 3.1: The effective potential (free energy density $F$ ) as a function of the VEV $v$ for $\mu_{B}=1, m=0.5$ and $g_{4}=0$.

The effect of quantum corrections at large $k$ will be to renormalise the mass in the symmetric phase and change the threshold value of the chemical potential at which the (second order) phase transition from the symmetric to the Higgsed phase occurs. This qualitative picture may change for finite $k$ when quantum corrections are large.

\subsubsection{Colour-flavour locked symmetry}

We have found a one-parameter family of gauge field solutions parametrised by the variables $\left(a_{1}, a_{2}\right)$, satisfying a constraint (3.20). Any given realisation breaks the $S U(2)$ gauge symmetry completely due to the scalar VEV which also breaks $U(1)_{B}$. However, the scalar VEV is left invariant by the diagonal combination of $U(1)_{B}$ and a $U(1)$ subgroup of the global $S U(2)$ colour rotations:

$$
U(1)_{B}:\langle\Phi\rangle \rightarrow e^{i \vartheta / 2}\langle\Phi\rangle, \quad U(1)_{C}: \Phi \rightarrow U(\vartheta) \Phi, \quad U(\vartheta) \equiv e^{i \vartheta \sigma_{3} / 2}
$$




\subsection{Spectrum of Fluctuations}

While the gauge fields do not transform under $U(1)_{B}$, they do transform under the global $U(1)_{C}$. The transformation acts on the background gauge fields $\left\langle A_{i}\right\rangle=\left\langle A_{i}^{(a)}\right\rangle t^{a}$ exactly as a rotation $(R)$ by a constant angle $\vartheta$ in the $x$-y plane:

$$
U(1)_{C}:\left(\begin{array}{l}
\left\langle A_{x}\right\rangle \\
\left\langle A_{y}\right\rangle
\end{array}\right) \rightarrow\left(\begin{array}{c}
U(\vartheta)\left\langle A_{x}\right\rangle U^{\dagger}(\vartheta) \\
U(\vartheta)\left\langle A_{y}\right\rangle U^{\dagger}(\vartheta)
\end{array}\right)=\left(\begin{array}{cc}
\cos \vartheta & -\sin \vartheta \\
\sin \vartheta & \cos \vartheta
\end{array}\right)\left(\begin{array}{l}
\left\langle A_{x}\right\rangle \\
\left\langle A_{y}\right\rangle
\end{array}\right) .
$$

Therefore the vacuum gauge configuration is invariant under a global $U(1)_{B+C+R}$ symmetry which can be viewed as a linear combination of global colour, flavour (or baryon number) and $S O(2)$ rotations in the $x-y$ plane.

The above observation has an important consequence. It implies that the ground state does not actually break rotational invariance ${ }^{1}$, since the action of rotations can be undone by a gauge transformation. This is naturally reflected in the expectation values of all gauge invariant operators built from field strengths. In particular, the expectation values of single trace operators built from the chromoelectric and chromomagnetic field strengths are independent of the spatial direction or spatial component in question:

$$
\left\langle\operatorname{Tr}\left(F_{0 i}\right)^{2}\right\rangle=-\frac{2 \pi^{3} v^{6}}{|k|^{3}}\left(\mu_{B}-\frac{v^{2} \pi}{|k|}\right), \quad\left\langle\operatorname{Tr}\left(F_{i j}\right)^{2}\right\rangle=-\frac{8 \pi^{2} v^{4}}{|k|^{3}}\left(\mu_{B}-\frac{v^{2} \pi}{|k|}\right)^{2} .
$$

\subsection{Spectrum of Fluctuations}

We now turn to the spectrum of quadratic fluctuations about the classical vacuum configuration. In the quantum theory this is reliable at weak coupling, i.e. $k \gg 1$ and $\mu_{B} \gg g_{4}$. The masses and dispersion relations derived in this section follow the technique described in Section 2.2.1 and so precise details into the derivations will be omitted from here on.

\footnotetext{
${ }^{1}$ This will be corroborated by the spectrum of physical fluctuations which we extract subsequently.
} 


\section{ROTON-PHONON EXCITATIONS IN CHERN-SIMONS MATTER THEORY AT FINITE DENSITY}

\subsubsection{The $k \rightarrow \infty$ theory}

It is useful to first recall the situation when the Chern-Simons fields are decoupled in the limit $k \rightarrow \infty$. In this limit we have a pure scalar field theory with a global $O(4) \supset S U(2) \times U(1)_{B}$ symmetry. A large enough chemical potential for $U(1)_{B}$ leads to Bose condensation via the scalar VEV,

$$
k \rightarrow \infty: \quad v^{2}=\frac{\mu_{B}^{2}-m^{2}}{2 g_{4}},
$$

and the weak coupling spectrum is readily obtained after diagonalising the matrix of quadratic fluctuations. There are four physical excitations corresponding to the four real scalar degrees of freedom with the following dispersion relations for the frequency $\omega$ as a function of the spatial momentum $\mathbf{p}$, where we have set $m=0$ for simplicity:

$$
\begin{aligned}
& \omega_{I( \pm)}^{2}=\mathbf{p}^{2}+3 \mu_{B}^{2} \pm \mu_{B} \sqrt{4 \mathbf{p}^{2}+9 \mu_{B}^{2}}, \\
& \omega_{I( \pm)}^{2}=\mathbf{p}^{2}+2 \mu_{B}^{2} \pm 2 \mu_{B} \sqrt{\mathbf{p}^{2}+\mu_{B}^{2}} .
\end{aligned}
$$

Two of these states are gapless ${ }^{1}$. Of the two, only one has a linear dispersion relation at low momentum and corresponds to the phonon mode while the other has a quadratic dependence on the spatial momentum,

$$
\omega_{I(-)}=\frac{|\mathbf{p}|}{\sqrt{3}}+\ldots, \quad \omega_{I I(-)}=\frac{\mathbf{p}^{2}}{2 \mu_{B}}+\ldots
$$

The presence of the second gapless mode with quadratic dependence on momentum implies that the Bose condensed ground state cannot be viewed as a superfluid, due to vanishing critical velocity according to the Landau criterion [208, 209]. This picture undergoes a qualitative change for finite large $k$.

\footnotetext{
${ }^{1}$ The chemical potential picks out a $U(1)_{B} \simeq S O(2) \subset O(4)$ and breaks the symmetry to $S O(3) \simeq S U(2)$. The scalar condensate spontaneously breaks both the $S U(2)$ and the $U(1)_{B}$, and the number of Goldstone bosons is fewer than the number of broken generators, as expected when relativistic invariance is absent [211, 212].
} 


\subsection{Spectrum of Fluctuations}

\subsubsection{Finite, large $k$}

For any finite value of $k$, the Chern-Simons gauge fields couple to the scalars. However, since the gauge fields are non-dynamical, the number of physical degrees of freedom remains unaltered and is given by the number of real scalars. To calculate the semi-classical spectrum we expand in fluctuations about the gauge and scalar VEVs,

$$
A_{\mu}=\left\langle A_{\mu}\right\rangle+\mathcal{A}_{\mu}, \quad \Phi=\langle\Phi\rangle+\delta \Phi, \quad \delta \Phi \equiv\left(\begin{array}{c}
\varphi_{1}+i \varphi_{2} \\
\varphi_{3}+i \varphi_{4}
\end{array}\right)
$$

where $\mathcal{A}_{\mu}$ and $\left\{\varphi_{i}\right\}(i=1, \ldots 4)$ are, respectively, the gauge field and matter fluctuations. Substituting these into the original action (3.2) and (3.4), and expanding to quadratic order in fluctuations,

$$
\begin{aligned}
\mathcal{L}^{(2)} & =\delta \Phi^{\dagger} \mathcal{D}_{\mu} \mathcal{D}^{\mu} \delta \Phi+\left\langle\Phi^{\dagger}\right\rangle \mathcal{A}_{\mu} \mathcal{D}^{\mu} \delta \Phi-\mathcal{D}_{\mu} \delta \Phi^{\dagger} \mathcal{A}_{\mu}\langle\Phi\rangle+\left\langle\Phi^{\dagger}\right\rangle \mathcal{A}_{\mu} \mathcal{A}^{\mu}\langle\Phi\rangle \\
& +\frac{k}{4 \pi} \epsilon^{\mu \nu \lambda} \operatorname{Tr}\left(\mathcal{A}_{\mu} \mathcal{D}_{\nu} \mathcal{A}_{\lambda}\right)-\frac{1}{2} \varphi_{j} \varphi_{k}\left\langle\frac{\partial^{2} V}{\partial \varphi_{j} \partial \varphi_{k}}\right\rangle
\end{aligned}
$$

Here $\mathcal{D}_{\mu}$ denotes the covariant derivative with respect to the background gauge field $\left\langle A_{\mu}\right\rangle$ :

$$
\mathcal{D}_{\mu} \delta \Phi \equiv \partial_{\mu} \delta \Phi+\left(\left\langle A_{\mu}\right\rangle+i \mu_{B} \delta_{\mu, 0}\right) \delta \Phi, \quad \mathcal{D}_{\mu} \mathcal{A}_{v} \equiv \partial_{\mu} \mathcal{A}_{v}+\left[\left\langle A_{\mu}\right\rangle, \mathcal{A}_{v}\right]
$$

The main point to note here is that, in the presence of the VEV for both scalars and gauge fields, all the fluctuations (matter and gauge) couple to each other at quadratic order. Due to the mixings, the physical degrees of freedom and their dispersion relations are not immediately obvious. In order to extract these, we first need to gauge-fix the action for the quadratic fluctuations. The gaugeunfixed action would yield a degenerate matrix with vanishing determinant. In the presence of background gauge fields and symmetry breaking scalar VEVs, it is natural to adopt an $R_{\xi}$ gauge, which is covariant with respect to the nonzero background gauge fields:

$$
\mathcal{L}^{(2)} \rightarrow \mathcal{L}^{(2)}+\mathcal{L}_{g f}, \quad \mathcal{L}_{g f}=\frac{1}{2 \xi} \operatorname{Tr}\left(\mathcal{D}_{\mu} \mathcal{A}^{\mu}-\xi\langle\Phi\rangle \delta \Phi^{\dagger}+\xi \delta \Phi\left\langle\Phi^{\dagger}\right\rangle\right)^{2}
$$




\section{ROTON-PHONON EXCITATIONS IN CHERN-SIMONS MATTER THEORY AT FINITE DENSITY}

The $R_{\xi}$ gauge above removes the derivative couplings between the would-be Goldstone modes and the gauge field fluctuations $\mathcal{A}_{\mu}$, and introduces a nontrivial mass matrix for them.

The determinant of the gauge-fixed fluctuation matrix then exhibits zeroes with both $\xi$-dependent and $\xi$-independent dispersion relations. The latter correspond to the physical states of the theory. In fact, these can be isolated by identifying the leading term in the large $\xi$ expansion of the determinant of fluctuations at fixed frequency and momentum.

\subsubsection{Physical states}

We have checked numerically that the physical states inferred from the procedure above are indeed $\xi$-independent. For the $S U(2)$ theory there are precisely four physical states corresponding to the two complex components of the scalar doublet, since the Chern-Simons gauge fields cannot contribute any additional physical, propagating degrees of freedom. The dispersion relations for these four physical states are given by the solutions to a quartic equation in $\left(\omega^{2}, \mathbf{p}^{2}\right)$,

$$
\omega^{8}+\mu_{B}^{2} C_{3} \omega^{6}+\mu_{B}^{4} C_{2} \omega^{4}+\mu_{B}^{6} C_{1} \omega^{2}+\mu_{B}^{8} C_{0}=0
$$

where the $\left\{C_{i}\right\}(i=0, \ldots 3)$ are functions of dimensionless variables,

$$
C_{i}=C_{i}\left(\frac{\mathbf{p}^{2}}{\mu_{B}^{2}}, \frac{g_{4}}{\mu_{B}}, \frac{m^{2}}{\mu_{B}^{2}}, k\right),
$$

whose explicit forms are given in the Appendix A.2.

The phonon mode: We first recall that the $U(1)_{B}$ global symmetry is spontaneously broken and the corresponding Goldstone mode is the phonon. Since the remaining broken symmetries are local, the phonon should be the only massless state. This is confirmed by solving for the spectrum using Equation 
3.37 at $\mathbf{p}=0$ which yields 1

$$
\begin{aligned}
& \mathbf{p}=\mathbf{0}: \quad \omega_{I(-)}=0, \quad \omega_{I(+)}=\sqrt{m^{2}+6 g_{4} v^{2}-\mu_{B}^{2}+\left(2\left|\mu_{B}\right|-\frac{\pi v}{|k|}\right)^{2}}, \\
& \omega_{I I(-)}=\frac{4 \pi}{|k|} v^{2}, \quad \omega_{I I(+)}=2\left|\mu_{B}\right| .
\end{aligned}
$$

As expected the gapless mode with a quadratic dispersion in the $k=\infty$ theory is lifted. It is then straightforward to find the velocity of the phonon mode. In the limit of small $\omega$ and $|\mathbf{p}|$ we identify the coefficients of the terms quadratic in $\omega$ and $\mathbf{p}$ in the polynomial (3.37). The resulting speed of sound is then,

$$
\begin{aligned}
& c_{s}=\left.\frac{d \omega}{d|\mathbf{p}|}\right|_{|\mathbf{p}| \rightarrow 0}=(1-y)^{1 / 2}\left(\frac{-15 y^{2}+12 y+\left(m^{2}+6 g_{4} v^{2}\right) \mu_{B}^{-2}-1}{y^{2}-4 y+\left(m^{2}+6 g_{4} v^{2}\right) \mu_{B}^{-2}-3}\right)^{1 / 2}, \\
& y \equiv \frac{\pi v^{2}}{\left|k \mu_{B}\right|} .
\end{aligned}
$$

The scalar VEV is given in Equation (3.22). In the massless limit $(m=0)$, the expression is purely a function of the dimensionless combination $\tilde{\mu}=\pi \mu_{B} /\left|g_{4} k\right|$ introduced earlier. In particular, the two distinct regimes of large $k$ (with $g_{4}$ fixed) and small $g_{4}$ (with $k$ fixed), which correspond to small and large $\tilde{\mu}$ respectively, are distinguished by two different limiting values for the speed of sound:

$$
\begin{array}{ll}
m=0, \quad \tilde{\mu} \ll 1: & c_{s}=\frac{1}{\sqrt{3}}\left(1+\frac{5 \tilde{\mu}}{12}-\frac{91 \tilde{\mu}^{2}}{96}+\ldots\right), \\
m=0, \quad \tilde{\mu} \gg 1: & c_{s}=\frac{1}{\sqrt{2}}\left(1-\frac{1}{8 \tilde{\mu}}+\frac{11}{128 \tilde{\mu}^{2}}+\ldots\right) .
\end{array}
$$

The limit of vanishing $g_{4}$ yields the free scalar field coupled to Chern-Simons gauge fields. In this limit the theory is conformal and therefore the speed of

\footnotetext{
${ }^{1}$ We follow the branches with the same nomenclature used for the $k=\infty$ theory. The subscripts $I(-)$ and $I I(-)$ refer to the gapless states in that theory with linear and quadratic dispersion relations, respectively.
} 


\section{ROTON-PHONON EXCITATIONS IN CHERN-SIMONS MATTER THEORY AT FINITE DENSITY}

sound is as expected for a scale-invariant theory in $2+1$ dimensions. This is a consistency check of the non-trivial Bose-condensed ground state we have discussed, stabilised by gauge field expectation values. It is also a consistency check on the dispersion relations for the semi-classical quadratic fluctuations. For non-zero scalar masses the phonon velocity is a non-trivial function of

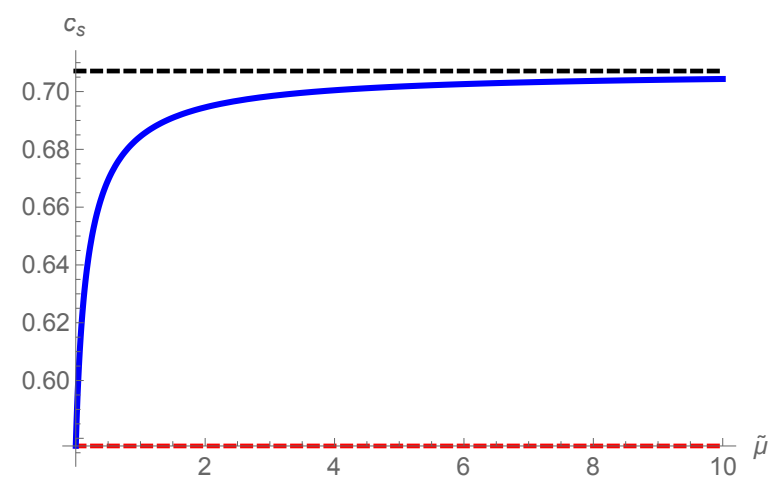

Figure 3.2: The solid blue curve shows the slope of the phonon dispersion relation at $\mathbf{p}=0$ as a function of $\tilde{\mu}=\pi\left|\mu_{B} / g_{4} k\right|$ for the massless theory, It interpolates between $c_{s}=1 / \sqrt{3}$ at small $\tilde{\mu}$ and the conformal value of $c_{s}=1 / \sqrt{2}$ when $g_{4}$ is taken to zero.

both $m$ and $\mu_{B}$. For instance, at large values of $k$ and all other parameters held fixed, we obtain

$$
c_{s}^{2}=\frac{\mu_{B}^{2}-m^{2}}{3 \mu_{B}^{2}-m^{2}}+\frac{\pi\left(5 \mu_{B}^{2}+m^{2}\right)\left(m^{2}-\mu_{B}^{2}\right)^{2}}{2\left|k \mu_{B}\right| g_{4}\left(m^{2}-\mu_{B}^{2}\right)^{2}}+O\left(1 / k^{2}\right) .
$$

The expression can be rewritten as a function of the two dimensionless parameters $\tilde{\mu}=\pi \mu_{B} / g_{4}|k|$ and $\tilde{m} \equiv \pi m / g_{4}|k|$.

Level crossing: The perturbative spectrum in the regime of small $\omega$ and $\mathbf{p}$ displays an interesting feature. This is a non-trivial consequence of crossing of energy levels which occurs as we tune the Chern-Simons level from $k=\infty$ to finite (large) values. This unavoidable crossing is between the phonon $\left(\omega_{I(-)}\right.$ branch) and the light state with energy $\omega_{I I(-)}$ which happens to be gapless with quadratic dispersion relation at $k=\infty$, but acquires a small gap $\sim 4 \pi v^{2} / k$ at 


\subsection{Spectrum of Fluctuations}

large $k$. The crossing is accompanied by off-diagonal mixings between these two fluctuations. In the low energy, long wavelength limit, where $\omega,|\mathbf{p}| \ll \mu_{B}$ (where we are ignoring $m$ for simplicity), it should suffice to focus attention on the two-level system comprising of the two lightest excitations. In this limit, the gapped modes only yield an overall multiplicative constant in the fluctuation determinant which takes the approximate form,

$$
\left(\omega^{2}-c_{s}^{2} \mathbf{p}^{2}\right)\left(\omega^{2}-\frac{\mathbf{p}^{4}}{4 \mu^{2}}-\delta\right)-\varepsilon \mathbf{p}^{4}=0
$$

The mixing term $\varepsilon$ scales as the inverse of the CS level, $\varepsilon \sim k^{-1}$, whilst the gap generated for the branch $\omega_{I I(-)}$ with quadratic dispersion scales as $\delta \sim k^{-2}$, both vanishing in the large $k$ limit. The mixing must necessarily be momentum dependent so that the gapless phonon mode persists as a Goldstone boson for the broken $U(1)_{B}$. At low momentum the leading such contribution scales as $\mathbf{p}^{4}$ (using Equation A.2)).
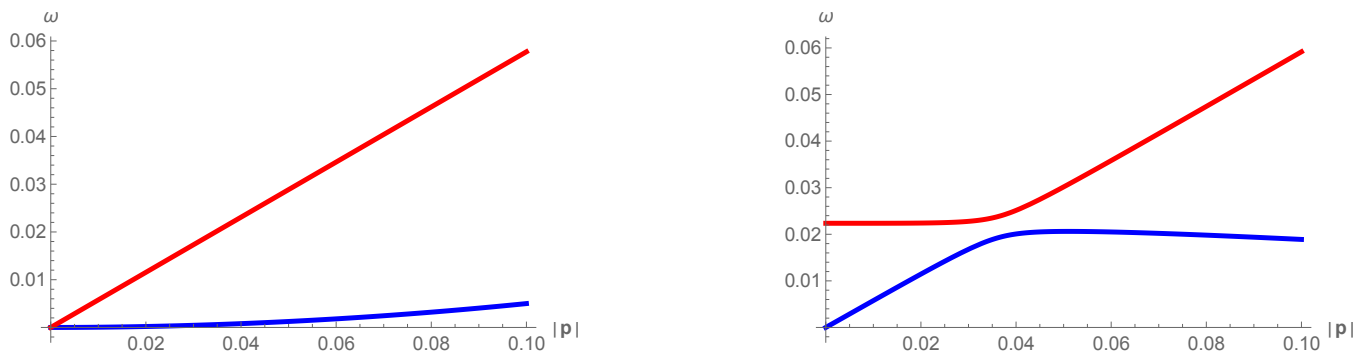

Figure 3.3: Left: The $k=\infty$ theory displays two gapless excitations with linear and quadratic dispersion relations. Right: At finite large $k$, the two modes potentially cross, and also mix. The diagonalised modes display a splitting and "repulsion" resulting in a local maximum in the phonon branch.

The new solutions to Equation (3.43) provide a qualitative description of the perturbed light spectrum at large, finite $k$. In particular, as shown in Figure (3.3), the two branches do not cross and the phonon branch displays a "maxon" or a local maximum in its dispersion relation. For non-zero $\varepsilon$, the two dispersion relations (viewed as functions of $\mathbf{p}^{2}$ ) have a branch-point in the complex plane. For small enough $\varepsilon$, the location of the maximum in $\omega_{I(-)}$ is 


\section{ROTON-PHONON EXCITATIONS IN CHERN-SIMONS MATTER THEORY AT FINITE DENSITY}

close to the putative interesection point of the two curves. The presence of this local maximum implies the existence of a "roton" minimum since all dispersion curves must eventually increase linearly at large $|\mathbf{p}|$ consistent with UV relativistic invariance.

\subsubsection{Roton minimum and complete spectrum}

Our main observation regarding the spectrum of excitations is that, for any (large) finite value of $k$ that is consistent with being in the semi-classical regime, the phonon branch always displays a roton minimum. At large $k$ and fixed $g_{4}$, the position of the maximum can be estimated quite easily. It sits close to the potential intersection point of the dispersion curves for $\omega_{I I(-)}$ and $\omega_{I(-)}$. In the large $k$ regime, the former is flat, $\omega_{I I(-)} \approx 4 \pi v^{2} /|k|$, while the latter is linear, $\omega_{I(-)} \approx|\mathbf{p}| / \sqrt{3}$, and their putative intersection is at

$$
k \gg 1, g_{4} \text { fixed }: \quad\left(\omega_{\max },|\mathbf{p}|_{\max }\right) \approx\left(\frac{4 \pi v^{2}}{|k|}, \frac{4 \pi v^{2} \sqrt{3}}{|k|}\right)
$$

On the other hand, the location of the roton minimum is more subtle. In the
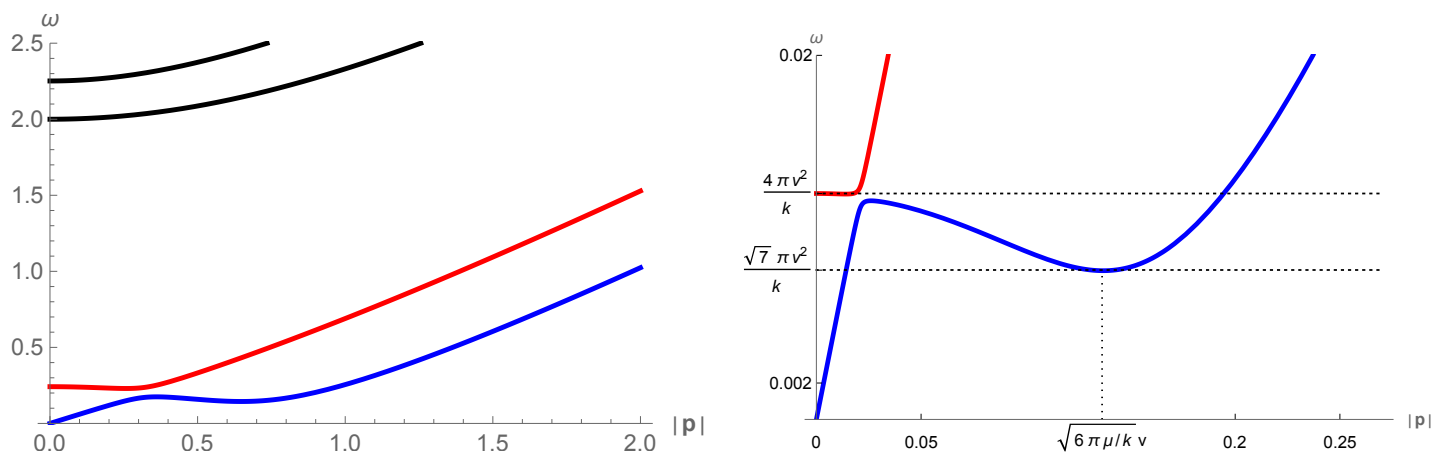

Figure 3.4: Left: The spectrum for $m=0$ with $\tilde{\mu}=\frac{\pi \mu_{B}}{g_{4} k}=\pi / 20$. Right: The two lightest states, including the phonon-roton branch (blue) with $\tilde{\mu}=\pi / 500$. The dotted lines indicate the large $k$ limiting values of the roton maximum and minimum.

large $k$ theory we expect the minimum to be located at parametrically small 
values close to the origin. In fact, it turns out that $\omega_{\text {rot }} \sim k^{-1}$ whilst $\mathbf{p}_{\text {rot }} \sim k^{-1 / 2}$. This can be checked by first performing the scaling

$$
\omega=\frac{1}{k} \omega, \quad \mathbf{p}=\frac{1}{\sqrt{k}} \rho,
$$

then substituting into the fluctuation determinant (A.2), and the expression for $\omega^{\prime}(\mathbf{p})$ by differentiating A.2. Subsequently, setting the determinant and $\omega^{\prime}(\mathbf{p})$ to zero, and then taking the large $k$ limit, we find (setting $m=0$ for simplicity):

$$
\begin{aligned}
& 3 \rho^{4}-24 \pi \mu_{B} \rho^{2} v^{2}+4 \mu_{B}^{2}\left(16 \pi^{2} v^{4}-\omega^{2}\right)=0, \\
& \rho^{4}-12 \pi \mu_{B} \rho^{2} v^{2}+4 \mu_{B}^{2}\left(16 \pi^{2} v^{4}-\omega^{2}\right)=0 .
\end{aligned}
$$

The solutions to these yield the roton minimum at large $k$ for the massless theory:

$$
k \gg 1: \quad\left(\omega_{\text {rot }},|\mathbf{p}|_{\text {rot }}\right)=\left(\frac{\sqrt{7} \pi v^{2}}{k}, \sqrt{\frac{6 \pi \mu}{k} v}\right),
$$

where the VEV is given by Equation 3.22 with $m=0$. The results for the roton minimum and maximum agree perfectly with the numerical curves for the phonon-roton branch at large $k$, displayed in Figure (3.4). The qualitative nature of the dispersion relations persists for all values of $m, \mu_{B}$ and $g_{4} k$. Figure (3.5) shows the relevant plots for one non-zero value of $m$.

Critical case with $g_{4}=m=0$ : A non-trivial aspect of the Bose condensed ground state is that all generic features of the spectrum of fluctuations persist even when $g_{4}=m=0$ (and $\mu_{B} \neq 0$ ) so that the classical theory is scale invariant. The determinant of physical fluctuations A.2 simplifies greatly, and the 


\section{ROTON-PHONON EXCITATIONS IN CHERN-SIMONS MATTER}

\section{THEORY AT FINITE DENSITY}
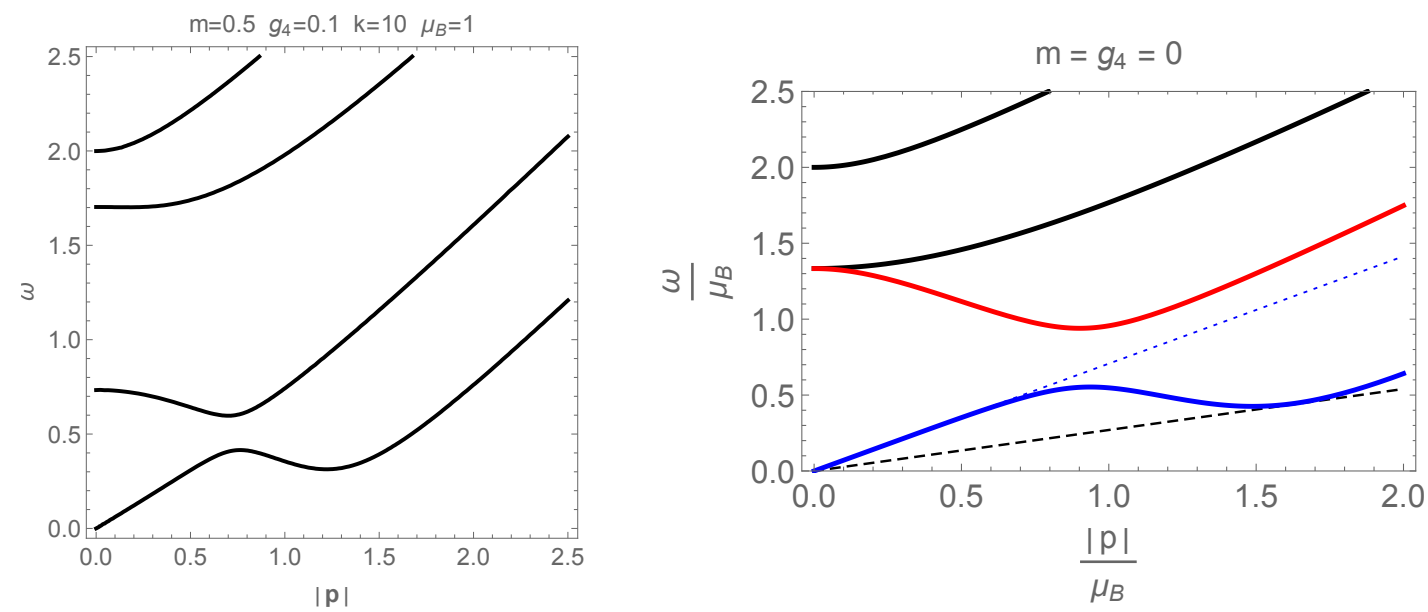

Figure 3.5: Left: The physical spectrum for the massive case. Right: In the scale invariant situation, the dispersion relations have a universal form, independent of $k$ and $\mu$. The dotted blue line with slope $1 / \sqrt{2}$ matches the phonon velocity at low momentum.

relevant dispersion relations are obtained from its zeroes:

$$
\begin{aligned}
& \tilde{p} \equiv \frac{|\mathbf{p}|}{\mu_{B}}, \quad \tilde{\omega} \equiv \frac{\omega}{\mu_{B}}, \\
& \tilde{p}^{8}-\tilde{p}^{6}\left(4 \tilde{\omega}^{2}+\frac{28}{9}\right)+\tilde{p}^{4}\left(6 \tilde{\omega}^{4}-\frac{4}{3} \tilde{\omega}^{2}+\frac{160}{81}\right)-\tilde{p}^{2}\left(4 \tilde{\omega}^{6}-12 \tilde{\omega}^{4}+\frac{992}{81} \tilde{\omega}^{2}-\frac{512}{81}\right) \\
& +\tilde{\omega}^{8}-\frac{68}{9} \tilde{\omega}^{6}+\frac{1408}{81} \tilde{\omega}^{4}-\frac{1024}{81} \tilde{\omega}^{2}=0 .
\end{aligned}
$$

At zero momentum the energies of the four physical states are:

$$
\omega_{I(-)}=0, \quad \omega_{I(+)}=\frac{4 \mu_{B}}{3}, \quad \omega_{I I(-)}=\frac{4 \mu_{B}}{3}, \quad \omega_{I I(+)}=2 \mu_{B},
$$

so that two of the massive states become degenerate, whilst the roton maximum and minimum are at

$$
\left(\omega_{\text {max }},|\mathbf{p}|_{\text {max }}\right)=\left(0.553 \mu_{B}, 0.937 \mu_{B}\right), \quad\left(\omega_{\text {rot }},|\mathbf{p}|_{\text {rot }}\right)=\left(0.426 \mu_{B}, 1.487 \mu_{B}\right)
$$


We expect these results to be stable against quantum corrections for large enough $k$, since $\frac{1}{k}$ is the only small parameter in the system. It is interesting and somewhat unexpected (given that the roton minimum is often attributed to the presence of a new scale) that the roton persists in the theory where the chemical potential is the only dimensionful scale.

\subsubsection{Landau critical velocity}

According to Landau's criterion, for a nonrelativistic superfluid flowing with velocity $v_{s}$ (with respect to a vessel or capillary), when the velocity exceeds a critical value [209] given by

$$
v_{\text {crit }}=\min _{|\mathbf{p}|}\left(\frac{\omega(\mathbf{p})}{|\mathbf{p}|}\right) \Longrightarrow \frac{\partial \omega}{\partial|\mathbf{p}|}=\frac{\omega}{|\mathbf{p}|},
$$

the fluid loses energy through dissipation and the superfluid phase can be wholly or partially destroyed e.g. by a condensate of rotons [213, 214]. In particular, [213] argues for the appearance, within superfluid ${ }^{4} \mathrm{He}$ flows, of a one dimensional periodic structure at rest with respect to the walls so that the superfluidity criterion is not violated. The Landau criterion is derived by boosting the Bose condensate in the ground state along a particular direction (say the $+x$-axis) with a velocity $v_{s}$, and considering excitations that could reduce or dissipate the energy of the moving condensate. In the frame where the condensate has velocity $v_{s}$, the energy of a backscattered non-relativistic excitation with momentum $\mathbf{p}$, causing dissipation from the condensate, must satisfy

$$
\omega(|\mathbf{p}|)-v_{s}|\mathbf{p}|<0
$$

where the second term is the result of transformation under the Galilean boost. The critical value of the superfluid velocity is then given as $v_{\text {crit }}=\min (\omega /|\mathbf{p}|)$. The arguments can also be carried out in the appropriate relativistic context (e.g. [209, 214]). The critical velocity is inferred from the slope of the straight line passing through the origin and tangent to the dispersion curve for the phonon-roton branch (see dashed black line in Figure (3.5)). 


\section{ROTON-PHONON EXCITATIONS IN CHERN-SIMONS MATTER THEORY AT FINITE DENSITY}

The behaviour of the critical velocity as a function of $\mu_{B} / g_{4} k$ in the massless theory is shown in Figure (3.6). At large $k$, the critical velocity vanishes as

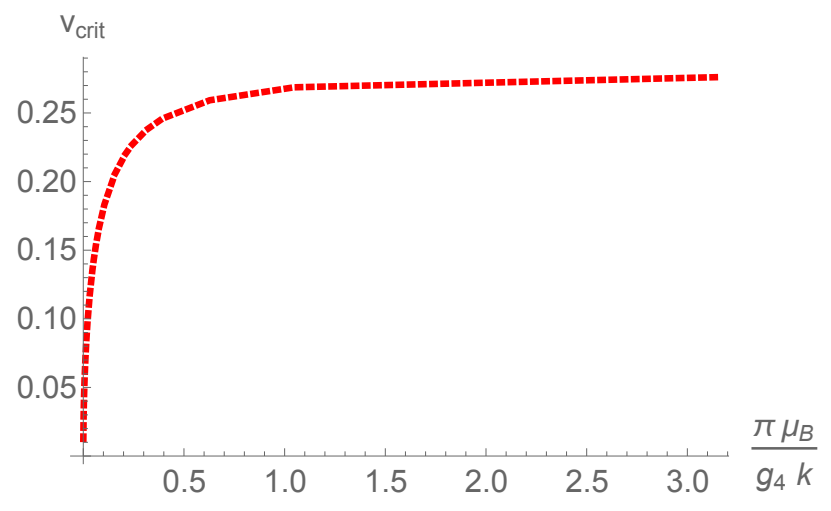

Figure 3.6: The Landau critical velocity as a function of the dimensionless parameter $\pi \mu_{B} / g_{4} k$ in the theory with $m=0$.

$1 / \sqrt{k}$, and approaches a constant value, $v_{\text {crit }} \approx 0.27$, in the theory with $g_{4}=0$.

\subsubsection{The $U(2)_{k}$ theory}

It is interesting to note the qualitative difference between $S U(2)$ and $U(2)$ gauge groups. In the latter case the $U(1)_{B}$ symmetry is gauged and the chemical potential is synonymous with a fixed background expectation value for the temporal component of the abelian gauge field. The classical vacuum equations are satisfied by the same configuration as in the $S U(2)$ theory. The condensates of the scalar and gauge fields break both the $S U(2)$ and $U(1)_{B}$ local symmetries to a diagonal $U(1)$. Since all symmetries are local we expect only massive physical states. We obtain the physical fluctuations by employing Coulomb gauge for the abelian gauge field, and retaining the covariant $R_{\xi}$ gauge-fixing for the $S U(2)$ part. The situation with $m=g_{4}=0$ suffices to demonstrate the existence of the gap. In this case, the dispersion relations of the four physical states can be obtained from the roots of the following poly- 
$\operatorname{nomial}$ in $(\tilde{\omega}, \tilde{p})=\left(\omega / \mu_{B},|\mathbf{p}| / \mu_{B}\right)$ :

$$
\begin{aligned}
& \tilde{p} \equiv \frac{|\mathbf{p}|}{\mu_{B}}, \quad \tilde{\omega} \equiv \frac{\omega}{\mu_{B}}, \\
& \tilde{p}^{8}-\tilde{p}^{6}\left(4 \tilde{\omega}^{2}+\frac{224}{81}\right)+\tilde{p}^{4}\left(6 \tilde{\omega}^{4}-\frac{64}{27} \tilde{\omega}^{2}+\frac{512}{243}\right)-\tilde{p}^{2}\left(4 \tilde{\omega}^{6}-\frac{352}{27} \tilde{\omega}^{4}+\frac{3328}{243} \tilde{\omega}^{2}-\frac{4096}{729}\right) \\
& +\tilde{\omega}^{8}-\frac{640}{81} \tilde{\omega}^{6}+\frac{4544}{243} \tilde{\omega}^{4}-\frac{10240}{729} \tilde{\omega}^{2}+\frac{16384}{59049} .
\end{aligned}
$$

Unlike the $S U(2)$ theory (Equation (3.48)), we see that $\tilde{\omega}=\tilde{p}=0$ is no longer a solution. All states are gapped at $\mathbf{p}=0$, with the energies given by $\tilde{\omega}^{2}=$ $16 / 9,16 / 9,(22 \pm 5 \sqrt{19}) 8 / 81$. The dispersion relations for non-zero $\mathbf{p}$ are shown in Figure 3.7.

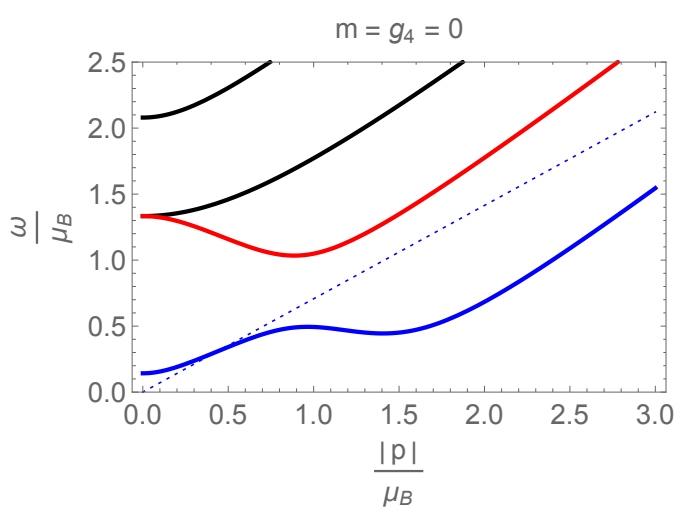

Figure 3.7: The semi-classical spectrum of the $U(2) \simeq S U(2) \times U(1)$ theory. All states are gapped. Nevertheless, the lightest state displays a roton-like minimum. The dotted blue line passing through the origin with slope $1 / \sqrt{2}$ is shown to emphasise the absence of phonon-like linear dispersion.

\subsection{The $S U(N>2)$ Case}

We now generalise the above analysis for Chern-Simons scalar theory with $S U(N)$ gauge group. We use lower case subscripts and superscripts, $(p, q, r \ldots)$ to label fundamental and anti-fundamental representation indices. The gauge 


\section{ROTON-PHONON EXCITATIONS IN CHERN-SIMONS MATTER THEORY AT FINITE DENSITY}

covariant derivative is defined to include the chemical potential as a timelike background gauge field:

$$
\left(D_{\mu}\right)_{p}^{q}=\delta_{p}^{q} \partial_{\mu}+\left(A_{\mu}\right)_{p}^{q}+i \mu_{B} \delta_{p}^{q} \delta_{\mu}^{0}
$$

For general $N$, it is useful to define the quartic coupling so that a consistent large $N$ limit can be taken if necessary. The potential contributions involving both gauge and scalar fields can be put together so that,

$$
\begin{aligned}
V_{C S}+V_{\text {scalar }} & =-\frac{k}{4 \pi} \frac{2}{3} \operatorname{Tr}\left(A_{\mu} A_{\nu} A_{\rho}\right) \epsilon^{\mu \nu \rho}-\Phi^{\dagger}\left(A^{\mu}+i \mu_{B} \eta^{\mu 0}\right)\left(A_{\mu}+i \mu_{B} \delta_{\mu}^{0}\right) \Phi \\
& +m^{2} \Phi^{\dagger} \Phi+\frac{g_{4}}{N}\left(\Phi^{\dagger} \Phi\right)^{2}
\end{aligned}
$$

Assuming that the scalar obtains a vacuum expectation value, we can always use $S U(N)$ gauge rotations to place the VEV in the $N$-th component,

$$
\left\langle\Phi_{p}\right\rangle=\sqrt{N} v \delta_{p, N}
$$

We have scaled out a factor of $\sqrt{N}$ in anticipation of the expected scaling in the large $N$ limit of vector models. In particular, the action for the matter fields should be $\mathcal{O}(N)$ in the large $N$ limit. The choice of scalar VEV leaves a residual $S U(N-1)$ gauge symmetry, which is then completely broken by the gauge field backgrounds in the ground state. In order to obtain the correct matrix equations of motion, we vary the action (3.54) subject to a tracelessness condition for $S U(N)$ gauge fields, implemented by Lagrange multipliers $\Lambda^{0,1,2}$ :

$$
V_{C S}+V_{\text {scalar }} \rightarrow V_{C S}+V_{\text {scalar }}+\Lambda^{\mu} \operatorname{Tr}\left(A_{\mu}\right)
$$

\subsubsection{Vacuum configuration}

The complete vacuum equations extremising the potential function are:

$$
\begin{aligned}
& -\frac{k}{4 \pi}\left[A_{\mu}, A_{v}\right] \epsilon^{\mu \nu \lambda}-\left\{\Phi \Phi^{\dagger},\left(A^{\lambda}+i \mu_{B} \eta^{\lambda 0} \mathbf{1}\right)\right\}+\Lambda^{\lambda} \mathbf{1}=0, \quad \operatorname{Tr} A_{\mu}=0 \\
& -\left(A_{\mu}\right)_{N}^{p}\left(A^{\mu}\right)_{p}^{N}+2 i \mu_{B}\left(A_{0}\right)_{N}^{N}+\left(m^{2}-\mu_{B}^{2}\right)+2 g_{4} v^{2}=0 .
\end{aligned}
$$


The matrix $\Phi \Phi^{\dagger}$ is a projector, and given that the scalar VEV can be rotated into the lowest component, it has only one non-zero element,

$$
\left(\Phi \Phi^{\dagger}\right)_{p}^{q}=N \delta_{p, N} \delta^{q, N} v^{2}
$$

The Lagrange multipliers $\left\{\Lambda^{\lambda}\right\}$ are determined by taking the trace of each of the respective equations of motion so that,

$$
\Lambda^{\lambda}=2 v^{2}\left[\left(A^{\lambda}\right)_{N}^{N}+i \mu_{B} \eta^{\lambda 0}\right]
$$

Following the hint provided by the $S U(2)$ vacuum solution, we take $A_{0}$ to be diagonal (note that residual $S U(N-1)$ rotations can be used to diagonalise an $(N-1) \times(N-1)$ block of $\left.A_{0}\right)$. It then follows that the commutator $\left[A_{x}, A_{y}\right]$ must be diagonal $\sim \operatorname{diag}(1,1, \ldots, 1-N)$. This is reminiscent of the $N$ dimensional representation of the $S U(2)$ algebra, where the off-diagonal ladder operators commute to yield a diagonal matrix. Motivated by this similarity, we find a simple solution for the Chern-Simons equations of motion:

$$
\begin{aligned}
& \left\langle A_{x}\right\rangle_{1}^{q}=i \alpha \delta^{q, 2}, \quad\left\langle A_{x}\right\rangle_{N}^{q}=i \alpha \sqrt{N-1} \delta^{q, N-1}, \\
& \left\langle A_{y}\right\rangle_{1}^{q}=\alpha \delta^{q, 2}, \quad\left\langle A_{y}\right\rangle_{N}^{q}=-\alpha \sqrt{N-1} \delta^{q, N-1}, \\
& \left\langle A_{x}\right\rangle_{p}^{q}=i \alpha\left(\sqrt{p} \delta^{q, p+1}+\sqrt{p-1} \delta^{q, p-1}\right), \quad p=2, \ldots N-1, \\
& \left\langle A_{y}\right\rangle_{p}^{q}=\alpha\left(\sqrt{p} \delta^{q, p+1}-\sqrt{p-1} \delta^{q, p-1}\right), \quad p=2, \ldots N-1, \\
& \left\langle A_{0}\right\rangle_{p}^{q}=i \beta\left(\frac{1}{N} \delta_{p}^{q}-\delta_{p, N} \delta^{q, N}\right), \quad p, q=1 \ldots N,
\end{aligned}
$$

where the constants $\alpha$ and $\beta$ are determined by the VEV and chemical potential as

$$
\alpha=\frac{\beta}{\sqrt{N}} \sqrt{\frac{\mu_{B}}{\beta}-\frac{N-1}{N}}, \quad \beta=\frac{v^{2}}{\kappa}, \quad \kappa \equiv \frac{k}{2 \pi N} .
$$




\section{ROTON-PHONON EXCITATIONS IN CHERN-SIMONS MATTER THEORY AT FINITE DENSITY}

The equation of motion for the scalar VEV (discarding the trivial extremum) is then given by

$$
-\frac{3}{\kappa^{2}}\left(1-\frac{1}{N}\right)^{2} v^{4}+v^{2}\left[2 g_{4}+\frac{4 \mu_{B}}{\kappa}\left(1-\frac{1}{N}\right)\right]-\left(\mu_{B}^{2}-m^{2}\right)=0 .
$$

Solving as a quadratic in $v^{2}$, only one solution is physical ${ }^{1}$ and matches smoothly onto the semi-classical $(\kappa \gg 1)$ limit:

$$
v^{2}=\frac{N \kappa}{3(N-1)}\left[\frac{g_{4} N \kappa}{(N-1)}+2 \mu_{B}-\sqrt{\left(\frac{g_{4} N \kappa}{N-1}+2 \mu_{B}\right)^{2}-3\left(\mu_{B}^{2}-m^{2}\right)}\right] .
$$

This agrees precisely with the result 3.22 for $N=2$ after we perform the rescalings, $v \rightarrow v / \sqrt{N}$ and $g_{4} \rightarrow g_{4} N$, required to match the conventions adopted in our analysis of the $S U(2)$ theory. It is also worth remarking that the $N \rightarrow \infty$ limit, keeping $\mathcal{K}$ and $g_{4}$ fixed, can be readily taken and $v$ remains finite in this limit.

For the free massless scalar coupled to Chern-Simons fields $\left(m=g_{4}=0\right)$, we obtain

$$
v^{2}=\kappa \frac{N \mu_{B}}{3(N-1)}, \quad \alpha=\frac{\mu_{B}}{3} \sqrt{\frac{2}{N-1}} .
$$

\subsubsection{Interpretation as quantum Hall droplet state}

The vacuum configuration breaks the $S U(N)$ gauge symmetry completely. The scalar field VEV also breaks the global $U(1)_{B}$ spontaneously and therefore the spectrum must yield a massless phonon mode. As seen previously in the $S U(2)$ theory, the classical background is left invariant by a diagonal combination of $U(1)_{B}$, global colour and spatial rotations. An $S O(2)$ rotation in the $x-y$ plane by an angle $\vartheta$, as in Equation (3.28), can be undone by a global gauge

\footnotetext{
${ }^{1}$ The second root yields $v^{2}>\kappa \mu_{B} N /(N-1)$ which would render $\alpha$ imaginary. In addition, this solution does not have a smooth $k \rightarrow \infty$ limit.
} 
transformation generated by the diagonal matrix $J_{3}$ :

$$
\begin{aligned}
& U(1)_{C}: \quad\left\langle A_{j}\right\rangle \rightarrow e^{i \vartheta J_{3}}\left\langle A_{j}\right\rangle e^{-i \vartheta J_{3},} \\
& J_{3} \equiv \operatorname{diag}\left(-\frac{N-1}{2},-\frac{N-3}{2},-\frac{N-5}{2} \ldots, \frac{N-3}{2}, \frac{N-1}{2}\right) .
\end{aligned}
$$

$J_{3}$ is the $N$ dimensional representation of one of the three generators of the $S U(2)$ algebra. The phase rotation of the scalar VEV generated by $J_{3}$ can clearly be compensated by a $U(1)_{B}$ transformation.

An interesting feature of the vacuum solution is that the hermitean matrices $i\left\langle A_{x}\right\rangle$ and $i\left\langle A_{y}\right\rangle$ provide a matrix realisation of coordinates on the noncommutative plane:

$$
\left[i\left\langle A_{x}\right\rangle, i\left\langle A_{y}\right\rangle\right]=2 i \alpha^{2}\left[\begin{array}{c|c}
1_{(N-1) \times(N-1)} & 0 \\
\hline 0 & 1-N
\end{array}\right],
$$

where the non-commutativity parameter is $2 \alpha^{2}$, as defined in Equation (3.61), and scales as $\alpha^{2} \sim 1 / N$ for large $N{ }^{1}$. Furthermore, it appears that the coordinates are restricted to within a disc or droplet:

$$
\left(i\left\langle A_{x}\right\rangle\right)^{2}+\left(i\left\langle A_{y}\right\rangle\right)^{2}=2 \alpha^{2} \operatorname{diag}(1,3,5, \ldots,(2 N-3),(N-1)) .
$$

The radius of the droplet is bounded in the large $N$ limit since $\alpha^{2} \sim 1 / N$ with limiting value

$$
\left.R_{\text {droplet }}\right|_{N \rightarrow \infty}=2 \beta \sqrt{\frac{\mu_{B}}{\beta}-1} .
$$

The algebra of matrices is closely related to that of harmonic oscillator creation and annihilation operators, when written in terms of the ladder operators:

$$
A^{ \pm}=i\left(\left\langle A_{x}\right\rangle \pm i\left\langle A_{y}\right\rangle\right)
$$

\footnotetext{
${ }^{1}$ It is tempting to look for solutions to the vacuum equations which are reducible and consist of irreducible lower dimensional blocks each satisfying the finite dimensional algebra implied by the vacuum conditions. We have not succeeded in finding any solutions of this type.
} 


\section{ROTON-PHONON EXCITATIONS IN CHERN-SIMONS MATTER THEORY AT FINITE DENSITY}

which, for any finite $N$, satisfy $\left(A^{+}\right)^{N}=\left(A^{-}\right)^{N}=0$. Precisely the same set of matrices were introduced to describe the fractional quantum Hall droplet by Polychronakos [93], building on the connection between abelian non-commutative Chern-Simons theory on the plane and the quantum Hall fluid [92], which we introduced in the Background chapter. The matrix model has also been shown to describe the low energy dynamics of vortices in $2+1$ dimensional YangMills-Higgs theory with a Chern-Simons term [190, 200]. In this picture, the matrices $i\left\langle A_{x}\right\rangle$ and $i\left\langle A_{y}\right\rangle$ parametrise the (non-commuting) coordinates of $N$ particles in the droplet. As Equation (3.67) indicates, the particles are placed in concentric circles of radius $\sim \sqrt{2 n-1}$ for $n=0,1,2, \ldots, N-1$. In the present context, the two matrices appear to deconstruct two dimensions (at large $N$ ) on top of the $2+1$ space-time dimensions in which the field theory is originally formulated.

Given the finite density "droplet" ground state for general $N$, we need to calculate the spectrum of fluctuations around it. This could be addressed in detail in future work. However, we can already make a few remarks. The spectrum must exhibit a massless state corresponding to the phonon arising from the spontaneous breaking of $U(1)_{B}$. In the droplet picture, physical excitations live only on the boundary of the quantum Hall droplet and are associated to area preserving deformations of the droplet boundary, subject to a Gauss' law constraint following from the Chern-Simons equations of motion [93]. These have a zero mode corresponding to rotations of the circular droplet ground state, which could naturally be identified with the phonon. In the language of the $N \times N$ matrices comprising the gauge field fluctuations, in an appropriate gauge (more precisely, unitary gauge), the excitations are encoded in the entries of the $N$-th row and column of gauge field fluctuations of $\mathcal{A}_{x}$ and $\mathcal{A}_{y}$, all other fluctuations correspond to pure gauge or "bulk" degrees of freedom of the droplet. It would be extremely interesting to flesh out this picture in detail and explore the implications of this interpretation for the spectrum of the theory for generic $N$, and in particular its large $N$ limit. 


\subsection{Vortices in Non-Abelian Chern-Simons Scalar Theories.}

Before we conclude this chapter, we address the possibility of a vortex solution in the background of the ground state we have seen above (Equations 3.20 , 3.22)). If vortices exist in this model, we expect them to be quite different from the ones we encountered in Chapter (2), since here we are considering a non-abelian theory.

Non-abelian vortices have been studied in a number of occasions in the past [215-218]. These are notably different to their abelian counterparts since the scalars appearing in those models are in the adjoint representation. This allows for the VEV to remain invariant under the center of the gauge group. If $z \in Z(G)$, where $Z(G)$ is the center of $G$, then by definition $z$ commutes with all elements in the group. Thus, if an adjoint scalar $\Phi$ has a non-zero VEV, then under a gauge transformation generated by $\mathrm{z}$, we have $\Phi \rightarrow z \Phi z^{\dagger}=\Phi z z^{\dagger}=\Phi$. In the above cases, the gauge group is $S U(N)$ with center $Z(S U(N))=\mathbb{Z}_{N}$. This means that the action of the gauge group $S U(N)$ on the adjoint scalars reduces to an $S U(N) / Z(S U(N))=S U(N) / \mathbb{Z}_{N}$. This gives rise to the possibility of a topological vortex, since $\pi_{1}\left(S U(N) / \mathbb{Z}_{N}\right)=\mathbb{Z}_{N}$, even though $\pi_{1}(S U(N))$ is trivial.

In contrast to these studies, we will be interested in fundamental scalars so this argument does not apply directly to our subject matter. However, due to the appearance of a non-zero chemical potential, we have found out that non-zero VEVs in the gauge sector come to play a role. Since the gauge fields are in the adjoint representation, this allows for the same type of argument for topological stability to reappear in a theory with fundamental matter. In the context of Yang-Mills theory, this type of vortex has been explored by Gorbar, Jia E Miransky [184].

Similarly to the situation that we encountered in Chapter (2), in the nonabelian case, we also observe a non-trivial ground state. This means that we can expect that there will be a circularly symmetric classical solution that interpolates between this ground state at infinity, and the zero fields configuration at the centre. And just as we elaborated in the previous paragraph, we ex- 


\section{ROTON-PHONON EXCITATIONS IN CHERN-SIMONS MATTER THEORY AT FINITE DENSITY}

pect to have two different types of topological vortices, depending on whether we are interested in the $\mathrm{U}(2)$ or $\mathrm{SU}(2)$ system. First, we focus our attention on the $U(2)$ theory.

\section{$3.6 \mathrm{U}(2)$ Theory}

In the $U(2)$ theory, we do not expect to find global vortices, since the global $U(1)$ symmetry in now gauged. In this model, we expect to find two types of vortices. The first one will be associated to a winding in the $U(1)$ and as such it is classified by $\pi_{1}(U(1))=\mathbb{Z}$. This leads to a charged, hence non-equilibrium, configuration, exactly as was the case in Chapter (2). For this reason, we need to introduce a background charge density in order to guarantee that the configuration is charge neutral. The second type of vortex owes its existence to the unusual form of the VEV (Equations (3.18) and (3.19)), namely the fact that the adjoint vector boson acquires non-zero expectation values. This vortex will be classified by

$$
\pi_{1}(S U(2) / Z(S U(2)))=\pi_{1}\left(S U(2) / \mathbb{Z}_{2}\right)=\mathbb{Z}_{2} .
$$

The action for the $U(2)$ theory can be written down as follows.

$$
S=S_{\mathrm{CS}}^{\mathrm{SU}(2)}+S_{\mathrm{CS}}^{\mathrm{U}(1)}+S_{\text {matter }},
$$

where

$$
\begin{aligned}
S_{\mathrm{CS}}^{\mathrm{SU}(2)} & =\frac{k}{4 \pi} \int d^{3} x \epsilon^{\mu v \rho} \operatorname{Tr}\left[A_{\mu} \partial_{\nu} A_{\rho}+\frac{2}{3} A_{\mu} A_{v} A_{\rho}\right], \\
S_{\mathrm{CS}}^{\mathrm{U}(1)} & =\frac{k^{\prime}}{4 \pi} \int d^{3} x \epsilon^{\mu v \rho} B_{\mu} \partial_{\nu} B_{\rho}, \\
S_{\text {matter }} & =-\int d^{3} x\left[\left(D_{\mu} \Phi\right)^{\dagger}\left(D^{\mu} \Phi\right)+V\left(\Phi^{\dagger} \Phi\right)\right], \\
D_{\mu} & =\partial_{\mu}+A_{\mu}+B_{\mu}+i \mu_{B} \delta_{\mu}^{0},
\end{aligned}
$$

where we have allowed for possibly different Chern-Simons levels for the abelian and non-abelian levels $k^{\prime}$ and $k$, respectively. This is consistent with the state- 
ments of duality that we saw in Chapter (1), Equations 1.149 1.151). We are restricting the potential here to be quartic

$$
V\left(\Phi^{\dagger} \Phi\right)=m^{2} \Phi^{\dagger} \Phi+g_{4}\left(\Phi^{\dagger} \Phi\right)^{2}
$$

Further, we require that the magnitude of the mass squared term is smaller than the magnitude of the chemical potential squared $m^{2}<\mu_{B}^{2}$, in order to guarantee symmetry breaking and the existence of the ground state that we studied in the previous sections. A sixth order monomial can also be included in the potential without changing the physics qualitatively, hence we choose to omit it here for simplicity. This leads to the classical equations of motion for the gauge sector

$$
\begin{array}{r}
\frac{k}{2 \pi} F_{\mu \nu}=\epsilon_{\mu \nu \lambda} J^{\lambda}, \\
\frac{k^{\prime}}{2 \pi} f_{\mu \nu}=\epsilon_{\mu \nu \lambda} \frac{1}{2} \operatorname{Tr}\left[\hat{J}^{\lambda}\right],
\end{array}
$$

where

$$
\begin{aligned}
F_{\mu \nu} & =\partial_{\mu} A_{v}-\partial_{\nu} A_{\mu}+\left[A_{\mu}, A_{\nu}\right], \quad f_{\mu \nu}=\partial_{\mu} B_{v}-\partial_{\nu} B_{\mu}, \\
\hat{J}^{\lambda} & =\sqrt{-g} g^{\lambda v}\left(\Phi\left(D_{\nu} \Phi\right)^{\dagger}-D_{\nu} \Phi \Phi^{\dagger}-2 i \mu_{B} \delta_{\nu}^{0} \Phi \Phi^{\dagger}\right), \\
J^{\lambda} & =\hat{J}^{\lambda}-\frac{1}{2} \operatorname{Tr}\left[\hat{J}^{\lambda}\right] \mathbb{I} \quad \Longrightarrow \quad \operatorname{Tr}\left[J^{\lambda}\right]=0 .
\end{aligned}
$$

The scalar equations arising from the action 3.71 are

$$
\left(D_{\mu}+i \mu \delta_{\mu}^{0}\right)\left[\sqrt{-g}\left(D^{\mu}+i \mu g^{\mu 0}\right)\right] \Phi=-\sqrt{-g} \frac{\delta V}{\delta \Phi^{\dagger}}
$$

Consider the ansatz

$$
\begin{aligned}
A_{\mu} & =i\left(a_{\mu}^{3} \sigma_{3}+a_{\mu}^{+} \sigma_{+}+a_{\mu}^{-} \sigma_{-}\right), \\
B_{\mu} & =i\left(a_{\mu} \mathbb{I}\right), \\
\Phi & =e^{i n \theta}\left(\begin{array}{c}
0 \\
\varphi(r)
\end{array}\right), \quad n \in \mathbb{Z}, \quad \varphi(r) \stackrel{r \rightarrow \infty}{\longrightarrow} v,
\end{aligned}
$$


where the matrices

$$
\begin{array}{rlrl}
\sigma_{3} & =\left[\begin{array}{cc}
1 & 0 \\
0 & -1
\end{array}\right], & \mathbb{I}=\left[\begin{array}{ll}
1 & 0 \\
0 & 1
\end{array}\right], \\
\sigma_{+}=\left[\begin{array}{ll}
0 & 1 \\
0 & 0
\end{array}\right], & \sigma_{-}=\left[\begin{array}{ll}
0 & 0 \\
1 & 0
\end{array}\right],
\end{array}
$$

are a basis of $U(2)$ that allows us to think of $a_{\mu}^{+}$as the complex conjugate of $a_{\mu}^{-}=\left(a_{\mu}^{+}\right)^{*}$. Further, restricting to a static, circularly symmetric solution, the ansatz becomes

$$
\begin{aligned}
a_{0 / \theta}^{+}=\tilde{g}_{0 / \theta}(r) e^{-i \theta+i \alpha}, & a_{0 / \theta}^{-}=\tilde{g}_{0 / \theta}(r) e^{+i \theta-i \alpha}, \\
a_{r}^{+}=\tilde{g}_{r}(r) e^{-i \theta+i\left(\alpha+\frac{\pi}{2}\right)}, & a_{r}^{-}=\tilde{g}_{r}(r) e^{+i \theta-i\left(\alpha+\frac{\pi}{2}\right),} \\
a_{\mu}^{(3)}=f_{\mu}(r), & f_{r}(r)=0, \\
a_{\mu}=a_{\mu}(r), & a_{r}(r)=0,
\end{aligned}
$$

where all of $\tilde{g}_{\mu} \in \mathbb{R}$. Note the $\frac{\pi}{2}$ shift of the phase of the $g_{r}$ component relative to the rest of the functions. This is analogous to how the vacuum components of $A_{\theta}$ and $A_{r}$ relate to one another. The phase factor $e^{i \alpha}$ has to do with the remaining global colour-flavour locked $U(1)$ symmetry in the system. Using the form of the ansatz above we have

$$
\Phi \Phi^{+}=\varphi(r)^{2}\left(\begin{array}{ll}
0 & 0 \\
0 & 1
\end{array}\right)=\varphi(r)^{2} \sigma_{-} \sigma_{+} .
$$


Thus, the system of differential equations simplifies to

$$
\begin{aligned}
\frac{k}{2 \pi}\left(f_{\theta}^{\prime}-2 \tilde{g}_{r} \tilde{g}_{\theta}\right) & =-r\left(a_{0}+\mu-f_{0}\right) \phi^{2}, \\
\frac{k}{2 \pi}\left(\tilde{g}_{\theta}^{\prime}+\tilde{g}_{r}-2 \tilde{g}_{r} f_{\theta}\right) & =r \tilde{g}_{0} \phi^{2}, \\
\frac{k^{\prime}}{2 \pi} a_{\theta}^{\prime} & =r\left(a_{0}+\mu-f_{0}\right) \phi^{2}-r J_{0}, \\
\frac{k}{2 \pi}\left(f_{0}^{\prime}+2 \tilde{g}_{0} \tilde{g}_{r}\right) & =\frac{1}{r}\left(a_{\theta}+n-f_{\theta}\right) \phi^{2}, \\
\frac{k}{2 \pi}\left(\tilde{g}_{0}^{\prime}+2 f_{0} \tilde{g}_{r}\right) & =\frac{1}{r} \tilde{g}_{\theta} \phi^{2}, \\
\frac{k^{\prime}}{2 \pi} a_{0}^{\prime} & =\frac{1}{r}\left(a_{\theta}+n-f_{\theta}\right) \phi^{2}, \\
\frac{k}{2 \pi}\left(\tilde{g}_{0}-2\left(f_{\theta} \tilde{g}_{0}-\tilde{g}_{\theta} f_{0}\right)\right) & =r \tilde{g}_{r} \phi^{2},
\end{aligned}
$$

where $J_{0}$ is determined by requiring that the system is neutral when evaluated

at the VEV. This implies that

$$
J_{0}=-\frac{\pi v^{2}}{k}\left(\frac{\mu k}{\pi}-v^{2}\right) .
$$

Inserting the ansatz 3.833 .85 and 3.883 .91 into the scalar equation of motion 3.82 we arrive at

$$
\begin{aligned}
\frac{1}{r} \frac{d}{d r}(r \varphi)+ & \varphi\left(\left(f_{0}-a_{0}-\mu\right)^{2}+\left|\tilde{g}_{0}\right|^{2}-\left(a_{r}-f_{r}\right)^{2}-\left|\tilde{g}_{r}\right|^{2}\right) \\
& +\varphi\left(-\frac{1}{r^{2}}\left(f_{\theta}-a_{\theta}-n\right)^{2}+\left|\tilde{g}_{\theta}\right|^{2}\right)=-\frac{\delta V}{\delta \Phi^{+}}
\end{aligned}
$$

We would like a solution that asymptotes to the ground state that we explored in Chapter (3), Equations 3.20, 3.22. This implies the following boundary 


\section{ROTON-PHONON EXCITATIONS IN CHERN-SIMONS MATTER THEORY AT FINITE DENSITY}

conditions

$$
\begin{array}{lr}
\varphi \stackrel{r \rightarrow \infty}{\longrightarrow} v, & \varphi \stackrel{r \rightarrow 0}{\longrightarrow} 0, \\
\tilde{g}_{\theta} \stackrel{r \rightarrow \infty}{\longrightarrow} r \frac{\pi v}{k} \sqrt{\frac{\mu k}{\pi}-v^{2},} & f_{\theta} \stackrel{r \rightarrow \infty}{\longrightarrow} 0, \\
\tilde{g}_{0} \stackrel{r \rightarrow \infty}{\longrightarrow} 0, & f_{0} \stackrel{r \rightarrow \infty}{\longrightarrow} \frac{\pi v^{2}}{k}, \\
a_{0} \stackrel{r \rightarrow \infty}{\longrightarrow} 0, & a_{\theta} \stackrel{r \rightarrow \infty}{\longrightarrow} 0,
\end{array}
$$

We have shown that a self-consistent circularly symmetric ansatz exists. The study of this boundary value problem has been left for future work. One can also arrive at the the $S U(2)$ equations of motion by requiring that the trace of the $U(2)$ equations of motion vanishes. This leads to a simplified system.

\subsection{Summary and Future Directions}

There are several immediate questions of interest that follow on from this chapter. The Bose condensed vacuum should have semi-classical vortex solutions, as we discussed in the preceeding section, and it would be interesting to understand their explicit construction given the non-abelian nature of the vacuum configuration. The ground state has a $U(1)$ colour-flavour locked global symmetry. A vortex solution that breaks this global symmetry will have an internal zero mode corresponding to a $U(1)$ moduli space of solutions. Such vortices in a (non-abelian) Higgs phase with non-commuting VEVs, carrying internal zero modes, have been encountered previously in different contexts [198, 219, 220]. The physical properties of such vortices and their role in the Bose-Fermi duality would be extremely interesting to explore.

So far in this exploration, we have arrived at a few intermediate results. We took the vacuum solutions in the $\mathrm{SU}(2)$ and $\mathrm{U}(2)$ theories found in this chapter and postulated the existence of vortex solutions asymptoting to these vacua. We found a consistent circularly symmetric ansatz and derived the equations 
of motion. Just as the ground state has an unbroken $U(1)$ symmetry, the solutions of the equations seem to also be parametrised by one real, compact parameter. Since the $S U(N)$ solutions correspond to a winding in the global symmetry, we expect that their energies will diverge in the infinite volume limit. However, in the $U(2)$ case the abelian symmetry is gauged so we expect the vortices in this model to be of finite energy. Further, due to the non-trivial vacuum structure, we expect these solutions to be topologically stable. We leave the numerical analysis of these solutions for further work.

The origin of roton-like minima is often attributed to long range interactions. The interpretation of the background VEVs as non-commuting "coordinates" for a quantum Hall droplet could thus provide a natural route to establish the existence of roton-like excitations ${ }^{1}$ for general $N>2$. In general, the computation of the spectrum of excitations and their dispersion relations about the Bose condensed ground state should be facilitated by the connection to the droplet picture of Polychronakos [93]. The goal would be to eventually understand the putative matching between the spectra of the bosonic theory at weak 't Hooft coupling $\left(\lambda_{B} \ll 1\right)$ and that of the dual critical fermion theory (coupled to Chern-Simons) at strong 't Hooft coupling $\left(\lambda_{F} \rightarrow 1\right)$. Perhaps the most puzzling aspect of this is the interpretation of the Higgsed ground state. When $\lambda_{F}=0$, and a $U(1)_{B}$ chemical potential is switched on in the critical theory, we do not expect fermion bilinears to condense (see e.g. Reference [222]). As $\lambda_{F}$ is increased from zero, it is conceivable that the effective potential for charged fermion bilinears carrying $U(1)_{B}$ favours a condensate, either for any non-zero $\lambda_{F}$ or at some critical value. It would be extremely interesting to understand the behaviour of the large $N$ effective potential for fermion bilinears for non-zero $\lambda_{F}$ and $\mu_{B}$.

A related question has recently been explored by Choudhury et al. [223] where Bose-Fermi duality at finite temperature and in the presence of scalar condensate has been established in the large $N$ 't Hooft limit. In order to reconcile their results with our ground state, we will need to understand the modification of the zero temperature finite density state, and in particular the back-

\footnotetext{
${ }^{1}$ See e.g. Castorina et al. [221] for a discussion of the relation between non-commutative field theory and roton excitations in bosonic and fermionic systems.
} 
ground gauge field VEVs, by any non-zero temperature. This is because the Euclidean finite temperature theory is effectively two dimensional at long distances and thus fluctuations in the phase of the scalar VEV are unsuppressed, which poses an unresolved puzzle.. It will be interesting to understand the fate of the phonon-roton mode at finite temperature and non-zero 't Hooft coupling in the Chern-Simons-scalar theory. 


\section{Conclusions}

In this thesis we have presented the results of a series of investigations focused around finite density Chern-Simons-matter theories. We found a symmetry broken (SB) phase in the $S U(2)$ theory that persists in the conformal phase of quartic coupling going to zero. The ground state exists both in the WilsonFisher fixed point and in the free scalar CS theory. This phase is interesting for several reasons. It is important in the context of Fermi-Bose duality, because up to date confirmations of the duality have not been made in the finite chemical potential regime. Such checks would require that this condensate is taken into account. Another reason why the symmetry broken phase is interesting is that it teaches us that giving VEVs to gauge fields does not necessarily break rotational invariance, since that can be mitigated by a colour-flavour locking mechanism. Thirdly, field theoretic techniques in conjunction with this configuration constitute a fundamental mechanism for the existence of roton excitations. This is important since rotons were first postulated to explain the heat capacity of superfluid helium [224] but no mechanism for their generation was proposed. Finally, this SB phase in the $S U(N)$ theory coincides with the noncommutative Chern-Simons description of the fractional quantum Hall effect.

We showed that vortex creation can be driven by chemical potential only. In other words, we found vortex solutions that do not require a symmetry breaking potential for their existence. Additionally, we found numerical evidence for BPS vortices, for which an analytic bound cannot be found in the traditional way, and derived an approximate corresponding bound. Finally, we found a self-consistent ansatz for non-abelian topological Chern-Simons vortices with fundamental matter that exist solely in the finite density regime. 
For future work, we wish to extend the computation of the quadratic spectrum of the $\mathrm{SU}(2)$ theory to the spectrum of the $\mathrm{SU}(\mathrm{N})$ theory. We suspect that the non-commutative Chern-Simons description is the gateway to achieving this and potentially solving the large $N S U(N)$ theory exactly. Additionally, we expect that this non-commutativity is somehow intrinsically linked to the fermionic description of the system. We would like to understand how this condensate manifests itself on the fermionic side of the duality. Is a fermion bilinear condensate $\bar{\psi} \psi$ responsible for the phase transition in the dual description? If yes, what is the precise mechanism? These are interesting questions, whose answers will help us gain a better understanding of Fermi-Bose duality. Furthermore, we would like to understand the solutions of the nonabelian equations of motion that we derived at the end of Chapter (3) and their role in the duality.

In conclusion, I would like to say that the last few years' contributions of high energy physics to the theory of condensed matter have been very exciting. I think that it is fascinating that ideas, models and theories that have arisen from the study of particle collisions, string theory and quantum gravity are now finding their place on the other extreme of the energy spectrum, in the world of ultracold physics. I am happy to have had the opportunity to contribute my infinitesimal share to this vast endeavour. 


\section{Appendix A}

\section{Determinant of fluctuation matrix for $S U(2)$}

The determinant for the physical fluctuations is given in terms of frequency $\omega$ and momentum $\mathbf{p}$ as,

$$
\omega^{8}+\mu_{B}^{2} C_{3} \omega^{6}+\mu_{B}^{4} C_{2} \omega^{4}+\mu_{B}^{6} C_{1} \omega^{2}+\mu_{B}^{8} C_{0}=0 .
$$




\section{A. DETERMINANT OF FLUCTUATION MATRIX FOR $S U(2)$}

Assuming $k>0, \mu_{B}>0$ the coefficients $\left\{C_{i}\right\}$ are (for general choice of signs, it is understood that $k$ and $\mu_{B}$ will be replaced by their absolute values below):

$$
\begin{aligned}
& C_{0}=\left(\frac{\mathbf{p}^{2}}{\mu_{B}^{2}}\right)^{4}+\left(\frac{\mathbf{p}^{2}}{\mu_{B}^{2}}\right)^{3}\left(\frac{m^{2}}{\mu_{B}^{2}}-1+\frac{6 g_{4} v^{2}}{\mu_{B}^{2}}+\frac{17 \pi^{2} v^{4}}{k^{2} \mu_{B}^{2}}-\frac{12 \pi v^{2}}{k \mu_{B}}\right)+\left(\frac{\mathbf{p}^{2}}{\mu_{B}^{2}}\right)^{2} \times \\
& \times\left(\frac{16 \pi^{2} m^{2} v^{4}}{k^{2} \mu_{B}^{4}}-\frac{12 \pi m^{2} v^{2}}{k \mu_{B}^{3}}+\frac{96 \pi^{2} g_{4} v^{6}}{k^{2} \mu_{B}^{4}}-\frac{72 \pi g_{4} v^{4}}{k \mu_{B}^{3}}+\frac{16 \pi^{4} v^{8}}{k^{4} \mu_{B}^{4}}-\frac{12 \pi^{3} v^{6}}{k^{3} \mu_{B}^{3}}-\frac{16 \pi^{2} v^{4}}{k^{2} \mu_{B}^{2}}\right. \\
& \left.+\frac{12 \pi v^{2}}{k \mu_{B}}\right)+\left(\frac{\mathbf{p}^{2}}{\mu_{B}^{2}}\right)\left(-\frac{384 \pi^{3} g_{4} v^{8}}{k^{3} \mu_{B}^{5}}+\frac{384 \pi^{2} g_{4} v^{6}}{k^{2} \mu_{B}^{4}}+\frac{960 \pi^{5} v^{10}}{k^{5} \mu_{B}^{5}}-\frac{1728 \pi^{4} v^{8}}{k^{4} \mu_{B}^{4}}\right. \\
& \left.-\frac{64 \pi^{3} m^{2} v^{6}}{k^{3} \mu_{B}^{5}}+\frac{832 \pi^{3} v^{6}}{k^{3} \mu_{B}^{3}}+\frac{64 \pi^{2} m^{2} v^{4}}{k^{2} \mu_{B}^{4}}-\frac{64 \pi^{2} v^{4}}{k^{2} \mu_{B}^{2}}\right) \\
& C_{1}=-4\left(\frac{\mathbf{p}^{2}}{\mu_{B}^{2}}\right)^{3}+\left(\frac{\mathbf{p}^{2}}{\mu_{B}^{2}}\right)^{2}\left(-5-\frac{3 m^{2}}{\mu_{B}^{2}}-\frac{18 g_{4} v^{2}}{\mu_{B}^{2}}-\frac{51 \pi^{2} v^{4}}{k^{2} \mu_{B}^{2}}+\frac{28 \pi v^{2}}{k \mu_{B}}\right)+ \\
& +\left(\frac{\mathbf{p}^{2}}{\mu_{B}^{2}}\right)\left(4-4 \frac{m^{2}}{\mu_{B}^{2}}-24 \frac{g_{4} v^{2}}{\mu_{B}^{2}}-\frac{192 \pi^{2} g_{4} v^{6}}{k^{2} \mu_{B}^{4}}+\frac{72 \pi g_{4} v^{4}}{k \mu_{B}^{3}}-\frac{32 \pi^{4} v^{8}}{k^{4} \mu_{B}^{4}}\right. \\
& \left.+\frac{76 \pi^{3} v^{6}}{k^{3} \mu_{B}^{3}}-\frac{32 \pi^{2} m^{2} v^{4}}{k^{2} \mu_{B}^{4}}-\frac{84 \pi^{2} v^{4}}{k^{2} \mu_{B}^{2}}+\frac{12 \pi m^{2} v^{2}}{k \mu_{B}^{3}}-\frac{28 \pi v^{2}}{k \mu_{B}}\right)-\frac{384 \pi^{2} g_{4} v^{6}}{k^{2} \mu_{B}^{4}} \\
& -\frac{64 \pi^{4} v^{8}}{k^{4} \mu_{B}^{4}}+\frac{256 \pi^{3} v^{6}}{k^{3} \mu_{B}^{3}}-\frac{64 \pi^{2} m^{2} v^{4}}{k^{2} \mu_{B}^{4}}-\frac{192 \pi^{2} v^{4}}{k^{2} \mu_{B}^{2}} \\
& C_{2}=6\left(\frac{\mathbf{p}^{2}}{\mu_{B}^{2}}\right)^{2}+\left(\frac{\mathbf{p}^{2}}{\mu_{B}^{2}}\right)\left(\frac{18 g_{4} v^{2}}{\mu_{B}^{2}}+\frac{51 \pi^{2} v^{4}}{k^{2} \mu_{B}^{2}}-\frac{20 \pi v^{2}}{k \mu_{B}}+\frac{3 m^{2}}{\mu_{B}^{2}}+13\right)+\frac{96 \pi^{2} g_{4} v^{6}}{k^{2} \mu_{B}^{4}} \\
& +\frac{24 g_{4} v^{2}}{\mu_{B}^{2}}+\frac{16 \pi^{4} v^{8}}{k^{4} \mu_{B}^{4}}-\frac{64 \pi^{3} v^{6}}{k^{3} \mu_{B}^{3}}+\frac{16 \pi^{2} m^{2} v^{4}}{k^{2} \mu_{B}^{4}}+\frac{116 \pi^{2} v^{4}}{k^{2} \mu_{B}^{2}}-\frac{16 \pi v^{2}}{k \mu_{B}}+\frac{4 m^{2}}{\mu_{B}^{2}}+12 \\
& C_{3}=-4\left(\frac{\mathbf{p}^{2}}{\mu_{B}^{2}}\right)-\frac{6 g_{B} v^{2}}{\mu_{B}^{2}}-\frac{17 \pi^{2} v^{4}}{k^{2} \mu_{B}^{2}}+\frac{4 \pi v^{2}}{k \mu_{B}}-\frac{m^{2}}{\mu_{B}^{2}}-7
\end{aligned}
$$




\section{References}

[1] H. Georgi and S. Glashow, "Unity of All Elementary Particle Forces", Phys. Rev. Lett. 32 (1974) 438-441.

[2] A. M. Polyakov, "Particle Spectrum in the Quantum Field Theory", JETP Lett. 20 (1974) 194-195. http://www.jetpletters.ac.ru/ps/1789/article_27297.shtml.

[3] G. 't Hooft, "Magnetic Monopoles in Unified Gauge Theories", Nucl. Phys. B 79 (1974) 276-284.

[4] A. M. Polyakov, "Quark Confinement and Topology of Gauge Groups", Nucl. Phys. B 120 (1977) 429-458.

[5] H. A. Kramers and G. H. Wannier, "Statistics of the Two-Dimensional Ferromagnet. Part I", Phys. Rev. 60 (Aug, 1941) 252-262.

[6] L. Onsager, "Crystal Statistics. I. A Two-Dimensional Model with an Order-Disorder Transition”, Phys. Rev. 65 (Feb, 1944) 117-149.

[7] S. R. Coleman, "The Quantum Sine-Gordon Equation as the Massive Thirring Model", Phys. Rev. D 11 (1975) 2088.

[8] A. Scott, F. Chu, and D. McLaughlin, "The soliton: A new concept in applied science”, Proceedings of the IEEE 61 no. 10, (1973) 1443-1483.

[9] W. E. Thirring, "A soluble relativistic field theory", Annals of Physics 3 no. 1, (Jan., 1958) 91-112. 


\section{REFERENCES}

[10] E. Witten, "Non-abelian bosonization in two-dimensions", Commun. Math. Phys. 92 (1984) 455-472.

[11] T. Nakanishi and A. Tsuchiya, "Level rank duality of WZW models in conformal field theory”, Commun. Math. Phys. 144 (1992) 351-372.

[12] S. G. Naculich and H. J. Schnitzer, "Duality Between SU(N)-k and SU(k)-N WZW Models", Nucl. Phys. B 347 (1990) 687-742.

[13] E. J. Mlawer, S. G. Naculich, H. A. Riggs, and H. J. Schnitzer, “Group level duality of WZW fusion coefficients and Chern-Simons link observables", Nucl. Phys. B352 (1991) 863-896.

[14] S. G. Naculich and H. J. Schnitzer, "Level-rank duality of the U(N) WZW model, Chern-Simons theory, and 2-D qYM theory", JHEP 06 (2007) 023, arXiv:hep-th/0703089.

[15] P. A. M. Dirac, "Quantised singularities in the electromagnetic field,", Proc. Roy. Soc. Lond. A A133 no. 821, (1931) 60-72.

[16] C. Montonen and D. I. Olive, "Magnetic Monopoles as Gauge Particles?", Phys. Lett. B 72 (1977) 117-120.

[17] H. Osborn, “Topological Charges for $\mathrm{N}=4$ Supersymmetric Gauge Theories and Monopoles of Spin 1", Phys. Lett. B 83 (1979) 321-326.

[18] E. Witten and D. I. Olive, "Supersymmetry Algebras That Include Topological Charges", Phys. Lett. B 78 (1978) 97-101.

[19] A. Sen, "Dyon - monopole bound states, selfdual harmonic forms on the multi - monopole moduli space, and SL $(2, Z)$ invariance in string theory", Phys. Lett. B 329 (1994) 217-221, arXiv : hep-th/9402032.

[20] C. Vafa and E. Witten, "A Strong coupling test of S duality", Nucl. Phys. B 431 (1994) 3-77, arXiv : hep-th/9408074. 
[21] N. Seiberg, "Exact results on the space of vacua of four-dimensional SUSY gauge theories", Phys. Rev. D 49 (1994) 6857-6863, arXiv:hep-th/9402044.

[22] N. Seiberg, "Electric-magnetic duality in supersymmetric non-Abelian gauge theories", Nucl. Phys. B 435 (1995) 129-146, arXiv: hep-th/9411149.

[23] N. Seiberg and E. Witten, "Electric-magnetic duality, monopole condensation, and confinement in $\mathcal{N}=2$ supersymmetric Yang-Mills theory", Nucl. Phys. B 426 (1994) 19-52, arXiv: hep-th/9407087. [Erratum: Nucl.Phys.B 430, 485-486 (1994)].

[24] O. Aharony, "IR duality in $\mathrm{d}=3 \mathrm{~N}=2$ supersymmetric $\operatorname{USp}(2 \mathrm{~N}(\mathrm{c}))$ and $\mathrm{U}(\mathrm{N}(\mathrm{c}))$ gauge theories", Phys. Lett. B 404 (1997) 71-76, arXiv:hep-th/9703215.

[25] A. Karch, "Seiberg duality in three-dimensions", Phys. Lett. B 405 (1997) 79-84, arXiv: hep-th/9703172.

[26] A. Giveon and D. Kutasov, "Seiberg Duality in Chern-Simons Theory", Nucl. Phys. B812 (2009) 1-11, arXiv:0808.0360.

[27] A. Sen, "Strong - weak coupling duality in four-dimensional string theory", Int. J. Mod. Phys. A 9 (1994) 3707-3750, arXiv:hep-th/9402002.

[28] J. H. Schwarz, "Evidence for nonperturbative string symmetries", Lett. Math. Phys. 34 (1995) 309-317, arXiv: hep-th/9411178.

[29] B. Sathiapalan, "Duality in Statistical Mechanics and String Theory", Phys. Rev. Lett. 58 (1987) 1597.

[30] C. Hull and P. Townsend, "Unity of superstring dualities", Nucl. Phys. B 438 (1995) 109-137, arXiv: hep-th/9410167.

[31] E. Witten, "String theory dynamics in various dimensions", Nucl. Phys. B 443 (1995) 85-126, arXiv: hep-th/9503124. 


\section{REFERENCES}

[32] L. Susskind, "The World as a hologram", J. Math. Phys. 36 (1995) 6377-6396, arXiv:hep-th/9409089.

[33] G. 't Hooft, "Dimensional reduction in quantum gravity", Conf. Proc. C 930308 (1993) 284-296, arXiv:gr-qc/9310026.

[34] O. Aharony, S. S. Gubser, J. M. Maldacena, H. Ooguri, and Y. Oz, "Large N field theories, string theory and gravity", Phys. Rept. 323 (2000) 183-386, arXiv: hep-th/9905111.

[35] J. M. Maldacena, "The Large N limit of superconformal field theories and supergravity", Int. J. Theor. Phys. 38 (1999) 1113-1133, arXiv:hep-th/9711200.

[36] M. A. Vasiliev, "More on equations of motion for interacting massless fields of all spins in (3+1)-dimensions", Phys. Lett. B 285 (1992) 225-234

[37] E. Sezgin and P. Sundell, "Holography in 4D (super) higher spin theories and a test via cubic scalar couplings", JHEP 07 (2005) 044, arXiv:hep-th/0305040.

[38] I. R. Klebanov and A. M. Polyakov, "AdS Dual of the Critical $O(N)$ Vector Model", Phys. Lett. B550 (2002) 213-219, arXiv:hep-th/0210114.

[39] S. Giombi, S. Minwalla, S. Prakash, S. P. Trivedi, S. R. Wadia, and X. Yin, "Chern-Simons Theory with Vector Fermion Matter", Eur. Phys. J. C72 (2012) 2112, arXiv: 1110.4386.

[40] O. Aharony, "Baryons, monopoles and dualities in Chern-Simons-matter theories", JHEP 02 (2016) 093, arXiv: 1512.00161.

[41] G. Gur-Ari and R. Yacoby, "Three Dimensional Bosonization From Supersymmetry", JHEP 11 (2015) 013, arXiv : 1507.04378 
[42] O. Aharony, G. Gur-Ari, and R. Yacoby, "Correlation Functions of Large N Chern-Simons-Matter Theories and Bosonization in Three Dimensions", JHEP 12 (2012) 028, arXiv : 1207.4593.

[43] S. Jain, S. Minwalla, and S. Yokoyama, "Chern Simons duality with a fundamental boson and fermion", JHEP 11 (2013) 037, arXiv: 1305.7235

[44] R. Dijkgraaf and E. Witten, "Topological Gauge Theories and Group Cohomology”, Commun. Math. Phys. 129 (1990) 393.

[45] S. Deser, R. Jackiw, and S. Templeton, "Topologically Massive Gauge Theories", Annals Phys. 140 (1982) 372-411. [Erratum: Annals Phys. 185, 406 (1988)].

[46] G. V. Dunne, "Aspects of Chern-Simons theory”, in Topological Aspects of Low-dimensional Systems: Proceedings, Les Houches Summer School of Theoretical Physics, Session 69: Les Houches, France, July 7-31 1998. arXiv:hep-th/9902115.

[47] E. Witten, "Quantum Field Theory and the Jones Polynomial", Commun. Math. Phys. 121 (1989) 351-399.

[48] D. Tong, "Lectures on the Quantum Hall Effect", 2016. arXiv: 1606.06687.

[49] E. H. Hall, "On a new action of the magnet on electric currents", American Journal of Mathematics 2 no. 3, (Sept., 1879) 287.

[50] K. von Klitzing, G. Dorda, and M. Pepper, "New method for high accuracy determination of the fine structure constant based on quantized Hall resistance", Phys. Rev. Lett. 45 (1980) 494-497.

[51] L. Landau, "Diamagnetismus der Metalle", Zeitschrift für Physik 64 no. 9-10, (Sept., 1930) 629-637.

[52] D. Yoshioka, The Quantum Hall Effect. Springer Berlin Heidelberg, Berlin, Heidelberg, 2002. 


\section{REFERENCES}

[53] S. M. Girvin, "The quantum hall effect: Novel excitations and broken symmetries", in Aspects topologiques de la physique en basse dimension. Topological aspects of low dimensional systems, pp. 53-175. Springer Berlin Heidelberg.

[54] D. C. Tsui, H. L. Stormer, and A. C. Gossard, "Two-dimensional magnetotransport in the extreme quantum limit", Phys. Rev. Lett. 48 (May, 1982) 1559-1562.

[55] R. Laughlin, “Anomalous quantum Hall effect: An Incompressible quantum fluid with fractionallycharged excitations", Phys. Rev. Lett. 50 (1983) 1395

[56] D. Tong, "TASI lectures on solitons: Instantons, monopoles, vortices and kinks", in Theoretical Advanced Study Institute in Elementary Particle Physics: Many Dimensions of String Theory. 6, 2005. arXiv:hep-th/0509216.

[57] N. J. Zabusky and M. D. Kruskal, "Interaction of "solitons" in a collisionless plasma and the recurrence of initial states", Phys. Rev. Lett. 15 (Aug, 1965) 240-243.

[58] M. Shifman, Advanced Topics in Quantum Field Theory: A Lecture Course. Cambridge University Press, Feb, 2012.

[59] A. Abrikosov, "The magnetic properties of superconducting alloys", Journal of Physics and Chemistry of Solids 2 no. 3, (Jan., 1957) 199-208.

[60] H. B. Nielsen and P. Olesen, "Vortex Line Models for Dual Strings", Nucl. Phys. B 61 (1973) 45-61.

[61] S. K. Paul and A. Khare, "Charged Vortices in Abelian Higgs Model with Chern-Simons Term”, Phys. Lett. B174 (1986) 420-422. [Erratum: Phys. Lett.B177,453(1986)].

[62] J. Hong, Y. Kim, and P. Y. Pac, "On the Multivortex Solutions of the Abelian Chern-Simons-higgs Theory", Phys. Rev. Lett. 64 (1990) 2230. 
[63] R. Jackiw and E. J. Weinberg, "Self-dual Chern-Simons Vortices", Phys. Rev. Lett. 64 (1990) 2234.

[64] R. Jackiw, K.-M. Lee, and E. J. Weinberg, "Self-dual Chern-Simons solitons", Phys. Rev. D 42 (1990) 3488-3499.

[65] J. M. Kosterlitz and D. J. Thouless, “Ordering, metastability and phase transitions in two-dimensional systems", Journal of Physics C: Solid State Physics 6 no. 7, (Apr, 1973) 1181-1203.

[66] V. Berezinsky, "Destruction of long range order in one-dimensional and two-dimensional systems having a continuous symmetry group. I. Classical systems", Sov. Phys. JETP 32 (1971) 493-500.

[67] V. Berezinsky, "Destruction of Long-range Order in One-dimensional and Two-dimensional Systems Possessing a Continuous Symmetry Group. II. Quantum Systems.”, Sov. Phys. JETP 34 no. 3, (1972) 610.

[68] E. Bogomolny, "Stability of Classical Solutions", Sov. J. Nucl. Phys. 24 (1976) 449.

[69] M. Prasad and C. M. Sommerfield, "An Exact Classical Solution for the 't Hooft Monopole and the Julia-Zee Dyon", Phys. Rev. Lett. 35 (1975) 760-762.

[70] M. E. Peskin, "Mandelstam 't Hooft Duality in Abelian Lattice Models", Annals Phys. 113 (1978) 122

[71] C. Dasgupta and B. Halperin, "Phase Transition in a Lattice Model of Superconductivity", Phys. Rev. Lett. 47 (1981) 1556-1560.

[72] K. A. Intriligator and N. Seiberg, "Mirror symmetry in three-dimensional gauge theories", Phys. Lett. B 387 (1996) 513-519, arXiv:hep-th/9607207.

[73] J. Murugan and H. Nastase, "A nonabelian particle-vortex duality", Phys. Lett. B 753 (2016) 401-405, arXiv: 1506.04090 [hep-th]. 


\section{REFERENCES}

[74] A. Karch and D. Tong, "Particle-Vortex Duality from 3d Bosonization", Phys. Rev. X6 no. 3, (2016) 031043, arXiv: 1606.01893

[75] A. Kapustin and M. J. Strassler, "On mirror symmetry in three-dimensional Abelian gauge theories", JHEP 04 (1999) 021, arXiv:hep-th/9902033.

[76] C. Burgess and B. P. Dolan, "Particle vortex duality and the modular group: Applications to the quantum Hall effect and other 2-D systems", Phys. Rev. B 63 (2001) 155309, arXiv: hep-th/0010246.

[77] J. Murugan, H. Nastase, N. Rughoonauth, and J. P. Shock, "Particle-vortex and Maxwell duality in the $A d S_{4} \times \mathbb{C P}^{3} / \mathrm{ABJM}$ correspondence", JHEP 10 (2014) 051, arXiv: 1404.5926 [ hep-th].

[78] F. Benini, C. Closset, and S. Cremonesi, "Comments on 3d Seiberg-like dualities", JHEP 10 (2011) 075, arXiv: 1108.5373.

[79] O. Aharony, S. S. Razamat, N. Seiberg, and B. Willett, "3d dualities from 4d dualities", JHEP 07 (2013) 149, arXiv : 1305.3924.

[80] O. Aharony and D. Fleischer, "IR Dualities in General 3d Supersymmetric SU(N) QCD Theories", JHEP 02 (2015) 162, arXiv: 1411.5475 .

[81] O. Aharony, S. Giombi, G. Gur-Ari, J. Maldacena, and R. Yacoby, "The Thermal Free Energy in Large N Chern-Simons-Matter Theories", JHEP 03 (2013) 121, arXiv: 1211.4843.

[82] A. P. Polychronakos, "Non-commutative Fluids", Prog. Math. Phys. 53 (2007) 109-159, arXiv:0706.1095.

[83] R. J. Szabo, "Quantum Field Theory on Noncommutative Spaces", Phys. Rept. 378 (2003) 207-299, arXiv : hep-th/0109162.

[84] M. R. Douglas and N. A. Nekrasov, "Noncommutative field theory", Rev. Mod. Phys. 73 (2001) 977-1029, arXiv : hep-th/0106048 
[85] R. Jackiw, S. Y. Pi, and A. P. Polychronakos, "Noncommuting Gauge Fields as a Lagrange Fluid", Annals Phys. 301 (2002) 157-173, arXiv:hep-th/0206014.

[86] H. S. Snyder, “Quantized Space-Time”, Phys. Rev. 71 (1947) 38-41.

[87] H. S. Snyder, "The Electromagnetic Field in Quantized Space-Time", Phys. Rev. 72 (1947) 68-71.

[88] A. Connes, Noncommutative geometry. Academic Press, 1994.

[89] E. Witten, "Noncommutative Geometry and String Field Theory", Nucl. Phys. B 268 (1986) 253-294.

[90] S. Sen and R. Holman, "A Noncommutative Geometry Model for Closed Bosonic Strings", Phys. Rev. Lett. 58 (1987) 1304.

[91] N. Seiberg and E. Witten, "String theory and noncommutative geometry", JHEP 09 (1999) 032, arXiv : hep-th/9908142.

[92] L. Susskind, "The Quantum Hall fluid and noncommutative Chern-Simons theory", arXiv: hep-th/0101029.

[93] A. P. Polychronakos, "Quantum Hall states as matrix Chern-Simons theory", JHEP 04 (2001) 011, arXiv : hep-th/0103013.

[94] S.-S. Chern and J. Simons, "Characteristic forms and geometric invariants", Annals Math. 99 (1974) 48-69.

[95] G. W. Moore and N. Read, "Nonabelions in the fractional quantum Hall effect", Nucl. Phys. B 360 (1991) 362-396.

[96] C. Nayak, S. H. Simon, A. Stern, M. Freedman, and S. Das Sarma, "Non-abelian anyons and topological quantum computation", Rev. Mod. Phys. 80 (Sep, 2008) 1083-1159.

[97] A. Kitaev, "Fault tolerant quantum computation by anyons", Annals Phys. 303 (2003) 2-30, arXiv:quant-ph/9707021. 


\section{REFERENCES}

[98] A. S. Schwarz, "Topological quantum field theories”, arXiv:hep-th/0011260.

[99] A. Achucarro and P. Townsend, "A Chern-Simons Action for Three-Dimensional anti-De Sitter Supergravity Theories", Phys. Lett. B 180 (1986) 89.

[100] S. Carlip, "Conformal field theory, $(2+1)$-dimensional gravity, and the BTZ black hole", Class. Quant. Grav. 22 (2005) R85-R124, arXiv:gr-qc/0503022.

[101] S. Deser, R. Jackiw, and S. Templeton, "Three-Dimensional Massive Gauge Theories", Phys. Rev. Lett. 48 (1982) 975-978.

[102] E. Witten, “Three-Dimensional Gravity Revisited”, arXiv:0706.3359 [hep-th].

[103] A. Zee, "Quantum hall fluids", in Field Theory, Topology and Condensed Matter Physics, pp. 99-153. Springer Berlin Heidelberg, 1995.

[104] G. W. Moore and N. Seiberg, "Taming the Conformal Zoo”, Phys. Lett. B 220 (1989) 422-430

[105] O. Aharony, G. Gur-Ari, and R. Yacoby, " $\mathrm{d}=3$ Bosonic Vector Models Coupled to Chern-Simons Gauge Theories", JHEP 03 (2012) 037, arXiv: 1110.4382 .

[106] H. Toda, "A topological proof of theorems of bott and borel-hirzeburch for homotopy groups of unitary groups", Memoirs of the College of Science, University of Kyoto. Series A: Mathematics 32 no. 1, (1959) 103-119.

[107] J. Dugundji, Topology. Prentice Hall, 1989.

[108] R. Jackiw, "Introduction to the Yang-Mills Quantum Theory", Rev. Mod. Phys. 52 (1980) 661-673. 
[109] A. P. Polychronakos, "On the Quantization of the Coefficient of the Abelian Chern-Simons Term”, Phys. Lett. B 241 (1990) 37-40.

[110] L. P. Kadanoff and G. Baym, Quantum Statistical Mechanics. CRC Press, Mar., 2018. https: // doi .org/10.1201/9780429493218.

[111] T. T. Wu and C. N. Yang, "Concept of Nonintegrable Phase Factors and Global Formulation of Gauge Fields", Phys. Rev. D 12 (1975) 3845-3857.

[112] T. T. Wu and C. N. Yang, "Dirac Monopole Without Strings: Monopole Harmonics”, Nucl. Phys. B 107 (1976) 365

[113] S. R. Coleman and B. R. Hill, "No More Corrections to the Topological Mass Term in QED in Three-Dimensions", Phys. Lett. B 159 (1985) 184-188.

[114] A. Khare, R. MacKenzie, and M. B. Paranjape, “On the Coleman-Hill theorem", Phys. Lett. B 343 (1995) 239-243, arXiv: hep-th/9408091.

[115] E. Witten, "Quantum field theory and the Jones polynomial", Communications in Mathematical Physics 121 no. 3, (Sept., 1989) 351-399.

[116] R. P. Feynman, A. R. Hibbs, and D. F. Styer, Quantum Mechanics and Path Integrals. 07, 2010.

[117] M. Laine and A. Vuorinen, Basics of Thermal Field Theory, vol. 925. Springer, 2016. arXiv: 1701.01554 [hep-ph].

[118] J. I. Kapusta, "Bose-einstein condensation, spontaneous symmetry breaking, and gauge theories", Phys. Rev. D 24 (Jul, 1981) 426-439.

[119] L. Landau, "The Theory of a Fermi Liquid", Sov. Phys. JETP 3 no. 6, (1957) 920.

[120] P. Drude, "Zur elektronentheorie der metalle”, Annalen der Physik 306 no. 3, (1900) 566-613 


\section{REFERENCES}

[121] P. Drude, "Zur elektronentheorie der metalle II. teil.

galvanomagnetische und thermomagnetische effecte", Annalen der Physik 308 no. 11, (1900) 369-402.

[122] I. I. Rabi, "Das freie Elektron im homogenen Magnetfeld nach der Diracschen Theorie", Zeitschrift für Physik 49 no. 7-8, (July, 1928) 507-511.

[123] V. Fock, "Bemerkung zur Quantelung des harmonischen Oszillators im Magnetfeld", Zeitschrift für Physik 47 no. 5-6, (May, 1928) 446-448.

[124] B. M. P. Frenkel' Ya. I., “Kvantovanie svobodnykh elektronov v magnitnom pole (Quantization of free electrons in a magnetic field)", Zh. Russ. Fiz.-Khim. Obshchestva 62 (1930) 485-494.

[125] B. I. Halperin, "Quantized hall conductance, current-carrying edge states, and the existence of extended states in a two-dimensional disordered potential", Phys. Rev. B 25 (Feb, 1982) 2185-2190.

[126] R. B. Laughlin, "Quantized hall conductivity in two dimensions", Phys. Rev. B 23 (May, 1981) 5632-5633.

[127] R. L. Willett, H. L. Stormer, D. C. Tsui, A. C. Gossard, and J. H. English, "Quantitative experimental test for the theoretical gap energies in the fractional quantum hall effect", Phys. Rev. B 37 (May, 1988) 8476-8479.

[128] P. Debye, "Zur theorie der spezifischen wärmen", Annalen der Physik 344 no. 14, (1912) 789-839.

[129] R. de Picciotto, M. Reznikov, M. Heiblum, V. Umansky, G. Bunin, and

D. Mahalu, "Direct observation of a fractional charge", Nature 389 (1997) 162-164.

[130] L. Saminadayar, D. C. Glattli, Y. Jin, and B. Etienne, "Observation of the e/3 fractionally charged laughlin quasiparticle", Phys. Rev. Lett. 79 (Sep, 1997) 2526-2529. 
[131] B. Halperin, "Theory of the quantized Hall conductance", Helv. Phys. Acta 56 (1983) 75-102.

[132] F. Wilczek, "Magnetic Flux, Angular Momentum, and Statistics", Phys. Rev. Lett. 48 (1982) 1144-1146.

[133] A. M. Polyakov, "Fermi-Bose Transmutations Induced by Gauge Fields", Mod. Phys. Lett. A 3 (1988) 325.

[134] J. Leinaas and J. Myrheim, "On the theory of identical particles", Nuovo Cim. B 37 (1977) 1-23.

[135] F. Wilczek, "Quantum mechanics of fractional-spin particles", Phys. Rev. Lett. 49 (Oct, 1982) 957-959.

[136] Y.-S. Wu, "General theory for quantum statistics in two dimensions", Phys. Rev. Lett. 52 (Jun, 1984) 2103-2106.

[137] E. Artin, "Theory of braids", The Annals of Mathematics 48 no. 1, (Jan., 1947) 101.

[138] E. Y. Andrei, G. Deville, D. C. Glattli, F. I. B. Williams, E. Paris, and B. Etienne, "Observation of a magnetically induced wigner solid", Phys. Rev. Lett. 60 (Jun, 1988) 2765-2768.

[139] E. Wigner, "On the interaction of electrons in metals", Phys. Rev. 46 (Dec, 1934) 1002-1011.

[140] E. Wigner, "Effects of the electron interaction on the energy levels of electrons in metals", Transactions of the Faraday Society 34 (1938) 678.

[141] Wen, Quantum field theory of many-body systems : from the origin of sound to an origin of light and electrons. Oxford University Press, Oxford New York, 2004.

[142] S. M. Girvin and A. H. MacDonald, "Off-diagonal long-range order, oblique confinement, and the fractional quantum hall effect", Phys. Rev. Lett. 58 (Mar, 1987) 1252-1255. 


\section{REFERENCES}

[143] S. C. Zhang, T. H. Hansson, and S. Kivelson, "Effective-Field-Theory Model for the Fractional Quantum Hall Effect”, Phys. Rev. Lett. 62 (Jan, 1989) 82-85.

[144] Z. F. Ezawa and A. Iwazaki, "Chern-simons gauge theories for the fractional-quantum-hall-effect hierarchy and anyon superconductivity", Phys. Rev. B 43 (Feb, 1991) 2637-2641.

[145] N. Read, "Order Parameter and Ginzburg-Landau Theory for the Fractional Quantum Hall Effect", Physical Review Letters 62 no. 1, (Jan., 1989) 86-89

[146] X.-G. Wen, "Topological orders and edge excitations in FQH states", Adv. Phys. 44 no. 5, (1995) 405-473, arXiv : cond-mat/9506066.

[147] X. G.wen, "Topological orders in rigid states”, International Journal of Modern Physics B $04(01,2012)$.

[148] X. G. Wen and A. Zee, "Classification of abelian quantum hall states and matrix formulation of topological fluids", Phys. Rev. B 46 (Jul, 1992) 2290-2301.

[149] Y. Aharonov and D. Bohm, "Significance of electromagnetic potentials in the quantum theory", Phys. Rev. 115 (1959) 485-491.

[150] R. G. Chambers, "Shift of an electron interference pattern by enclosed magnetic flux", Phys. Rev. Lett. 5 (Jul, 1960) 3-5.

[151] R. Willett, J. Eisenstein, H. Stormer, D. Tsui, A. Gossard, and J. English, "Observation of an even-denominator quantum number in the fractional quantum Hall effect”, Phys. Rev. Lett. 59 (1987) 1776-1779.

[152] J. K. Jain, “Composite-fermion approach for the fractional quantum hall effect”, Phys. Rev. Lett. 63 (Jul, 1989) 199-202.

[153] S. Mandelstam, "Vortices and Quark Confinement in Nonabelian Gauge Theories", Phys. Rept. 23 (1976) 245-249. 
[154] Y. Nambu, "Strings, monopoles, and gauge fields", Phys. Rev. D 10 (Dec, 1974) 4262-4268.

[155] A. Polyakov, "Quark confinement and topology of gauge theories", Nuclear Physics B 120 no. 3, (Mar., 1977) 429-458.

[156] N. Seiberg and E. Witten, "Monopoles, duality and chiral symmetry breaking in $\mathrm{n}=2$ supersymmetric QCD", Nuclear Physics B 431 no. 3, (Dec., 1994) 484-550.

[157] C. H. Taubes, "Arbitrary N-vortex Solutions to the First Order Ginzburg-Landau Equations", Communications in Mathematical Physics 72 no. 3, (Oct., 1980) 277-292.

[158] C. H. Taubes, "On the equivalence of the first and second order equations for gauge theories", Communications in Mathematical Physics 75 no. 3, (Oct., 1980) 207-227.

[159] B. Plohr, "The behavior at infinity of isotropic vortices and monopoles", Journal of Mathematical Physics 22 no. 10, (Oct., 1981) 2184-2190.

[160] M. Berger and Y. Chen, "Symmetric vortices for the ginzberg-landau equations of superconductivity and the nonlinear desingularization phenomenon", Journal of Functional Analysis 82 no. 2, (Feb., 1989) 259-295.

[161] J. Kierzenka and L. F. Shampine, "A BVP solver based on residual control and the maltab PSE", ACM Transactions on Mathematical Software (TOMS) 27 no. 3, (Sept., 2001) 299-316.

[162] K. G. Wilson and M. E. Fisher, "Critical exponents in 3.99 dimensions", Phys. Rev. Lett. 28 240-243.

[163] D. G. Barci, C. D. Fosco, and L. E. Oxman, “On bosonization in three-dimensions”, Phys. Lett. B375 (1996) 267-272, arXiv:hep-th/9508075. 


\section{REFERENCES}

[164] C. P. Burgess and F. Quevedo, "Bosonization as duality", Nucl. Phys. B421 (1994) 373-390, arXiv: hep-th/9401105.

[165] E. H. Fradkin and F. A. Schaposnik, "The Fermion - boson mapping in three-dimensional quantum field theory", Phys. Lett. B 338 (1994) 253-258, arXiv: hep-th/9407182.

[166] S. G. Naculich, H. A. Riggs, and H. J. Schnitzer, "Group Level Duality in WZW Models and Chern-Simons Theory", Phys. Lett. B246 (1990) 417-422

[167] M. Camperi, F. Levstein, and G. Zemba, “The Large N Limit of Chern-Simons Gauge Theory", Phys. Lett. B247 (1990) 549-554.

[168] R. Feynman, The Character of Physical Law. MIT Press, 1965.

[169] R. D. Pisarski and S. Rao, "Topologically massive chromodynamics in the perturbative regime", Phys. Rev. D 32 (Oct, 1985) 2081-2096

[170] I. Tyutin, "Gauge Invariance in Field Theory and Statistical Physics in Operator Formalism", arXiv:0812.0580 [hep-th].

[171] C. Becchi, A. Rouet, and R. Stora, "The Abelian Higgs-Kibble Model. Unitarity of the S Operator", Phys. Lett. B 52 (1974) 344-346.

[172] C. Becchi, A. Rouet, and R. Stora, "Renormalization of Gauge Theories", Annals Phys. 98 (1976) 287-321.

[173] V. Nair and A. Polychronakos, "On Level quantization for the noncommutative Chern-Simons theory", Phys. Rev. Lett. 87 (2001) 030403, arXiv: hep-th/0102181.

[174] D. Bak, K.-M. Lee, and J.-H. Park, "Chern-Simons theories on noncommutative plane", Phys. Rev. Lett. 87 (2001) 030402, arXiv: hep-th/0102188. 
[175] J. H. Brodie, L. Susskind, and N. Toumbas, "How Bob Laughlin tamed the giant graviton from Taub - NUT space", JHEP 02 (2001) 003, arXiv:hep-th/0010105.

[176] I. Bena and A. Nudelman, "On the stability of the quantum Hall soliton", JHEP 12 (2000) 017, arXiv: hep-th/0011155.

[177] S. S. Gubser and M. Rangamani, "D-brane dynamics and the quantum Hall effect", JHEP 05 (2001) 041, arXiv : hep-th/0012155.

[178] R. Banerjee, B. Chakraborty, S. Ghosh, P. Mukherjee, and S. Samanta, "Topics in Noncommutative Geometry Inspired Physics", Found. Phys. 39 (2009) 1297-1345, arXiv:0909.1000 [hep-th].

[179] G. Dunne and R. Jackiw, “"peierls substitution” and chern-simons quantum mechanics", Nuclear Physics B - Proceedings Supplements 33 no. 3, (Nov., 1993) 114-118.

[180] G. V. Dunne, R. Jackiw, and C. A. Trugenberger, “"'topological” (chern-simons) quantum mechanics", Phys. Rev. D 41 (Jan, 1990) 661-666.

[181] P. A. Horvathy and P. Zhang, "Vortices in (abelian) Chern-Simons gauge theory", Phys. Rept. 481 (2009) 83-142, arXiv:0811.2094.

[182] S. P. Kumar, D. Roychowdhury, and S. Stratiev, "Roton-phonon excitations in Chern-Simons matter theory at finite density", JHEP 12 (2018) 116, arXiv: 1806.06976.

[183] V. P. Gusynin, V. A. Miransky, and I. A. Shovkovy, "Spontaneous rotational symmetry breaking and roton - like excitations in gauged $\sigma$-model at finite density", Phys. Lett. B581 (2004) 82-92, arXiv: hep-ph/0311025. [Erratum: Phys. Lett.B734,407(2014)].

[184] E. V. Gorbar, J. Jia, and V. A. Miransky, "Vortices in gauge models at finite density with vector condensates", Phys. Rev. D73 (2006) 045001, arXiv:hep-ph/0512203. 


\section{REFERENCES}

[185] N. Seiberg, T. Senthil, C. Wang, and E. Witten, "A Duality Web in 2+1 Dimensions and Condensed Matter Physics", Annals Phys. 374 (2016) 395-433, arXiv: 1606.01989.

[186] J. Murugan and H. Nastase, "Particle-vortex duality in topological insulators and superconductors", JHEP 05 (2017) 159, arXiv:1606.01912.

[187] S. Jain, S. Minwalla, T. Sharma, T. Takimi, S. R. Wadia, and

S. Yokoyama, "Phases of large $N$ vector Chern-Simons theories on $S^{2} \times S^{1}$ ", JHEP 09 (2013) 009, arXiv : 1301.6169.

[188] T. Takimi, "Duality and Higher Temperature Phases of Large N

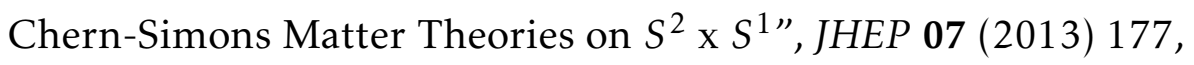
arXiv: 1304.3725 .

[189] M. Geracie, M. Goykhman, and D. T. Son, "Dense Chern-Simons Matter with Fermions at Large N", JHEP 04 (2016) 103, arXiv: 1511.04772.

[190] D. Tong and C. Turner, "Quantum Hall Effect in Supersymmetric Chern-Simons Theories", Phys. Rev. B92 no. 23, (2015) 235125, arXiv: 1508.00580 .

[191] S. Bolognesi, "Large $N, \mathbb{Z}_{N}$ Strings and Bag Models", Nucl. Phys. B730 (2005) 150-163, arXiv: hep-th/0507286.

[192] S. Bolognesi, "Multi-monopoles and Magnetic Bags", Nucl. Phys. B752 (2006) 93-123, arXiv: hep-th/0512133

[193] S. Bolognesi and S. B. Gudnason, "A Note on Chern-Simons solitons: A Type III vortex from the wall vortex", Nucl. Phys. B805 (2008) 104-123, arXiv:0711.3803.

[194] A. Chodos, R. L. Jaffe, K. Johnson, C. B. Thorn, and V. F. Weisskopf, "New extended model of hadrons", Phys. Rev. D 9 (Jun, 1974) 3471-3495. 
[195] J. I. Kapusta, "Bose-Einstein Condensation, Spontaneous Symmetry Breaking, and Gauge Theories", Phys. Rev. D 24 (1981) 426-439.

[196] R. A. Rosen, "Phase Transitions of Charged Scalars at Finite Temperature and Chemical Potential", JHEP 12 (2010) 024, arXiv: 1009.0752.

[197] O. Aharony, O. Bergman, D. L. Jafferis, and J. Maldacena, "N=6 superconformal Chern-Simons-matter theories, M2-branes and their gravity duals", JHEP 10 (2008) 091, arXiv:0806.1218 [hep-th].

[198] R. Auzzi and S. P. Kumar, "Non-Abelian Vortices at Weak and Strong Coupling in Mass Deformed ABJM Theory", JHEP 10 (2009) 071, arXiv:0906.2366.

[199] J. Hong, Y. Kim, and P. Y. Pac, "Multivortex solutions of the Abelian Chern-Simons-Higgs theory", Physical Review Letters 64 no. 19, (May, 1990) 2230-2233.

[200] D. Tong, “A Quantum Hall fluid of vortices”, JHEP 02 (2004) 046, arXiv:hep-th/0306266.

[201] J. Maldacena and A. Zhiboedov, “Constraining Conformal Field Theories with A Higher Spin Symmetry", J. Phys. A46 (2013) 214011, arXiv: 1112.1016.

[202] J. Maldacena and A. Zhiboedov, "Constraining conformal field theories with a slightly broken higher spin symmetry", Class. Quant. Grav. 30 (2013) 104003, arXiv: 1204.3882.

[203] S. Jain, M. Mandlik, S. Minwalla, T. Takimi, S. R. Wadia, and

S. Yokoyama, "Unitarity, Crossing Symmetry and Duality of the S-matrix in large N Chern-Simons theories with fundamental matter", JHEP 04 (2015) 129, arXiv : 1404.6373.

[204] Y. Dandekar, M. Mandlik, and S. Minwalla, "Poles in the S-Matrix of Relativistic Chern-Simons Matter theories from Quantum Mechanics", JHEP 04 (2015) 102, arXiv : 1407. 1322. 


\section{REFERENCES}

[205] S. R. Coleman, "There are no Goldstone bosons in two-dimensions", Commun. Math. Phys. 31 (1973) 259-264.

[206] N. D. Mermin and H. Wagner, "Absence of Ferromagnetism or Antiferromagnetism in One- or Two-Dimensional Isotropic Heisenberg Models", Phys. Rev. Lett. 17 (1966) 1133-1136.

[207] G. Gur-Ari, S. A. Hartnoll, and R. Mahajan, "Transport in Chern-Simons-Matter Theories", JHEP 07 (2016) 090, arXiv: 1605.01122.

[208] L. D. Landau, "The theory of superfuidity of helium II", J. Phys.(USSR) 5 (1941) 71-100.

[209] A. Schmitt, "Introduction to Superfluidity", Lect. Notes Phys. 888 (2015) pp.1-155, arXiv: 1404.1284.

[210] S. Minwalla and S. Yokoyama, "Chern Simons Bosonization along RG Flows”, JHEP 02 (2016) 103, arXiv : 1507.04546.

[211] H. B. Nielsen and S. Chadha, "On How to Count Goldstone Bosons", Nucl. Phys. B105 (1976) 445-453.

[212] H. Watanabe and H. Murayama, "Unified Description of Nambu-Goldstone Bosons without Lorentz Invariance", Phys. Rev. Lett. 108 (2012) 251602, arXiv: 1203.0609.

[213] L. P. Pitaevskii, "Layered structure of "He with supercritical motion", JETP 39(9) (1984) 511-514. http://www.jetpletters.ac.ru/ps/1301/article_19659.shtml.

[214] D. N. Voskresenskii, "Condensate with finite momentum in a moving medium", JETP 77(6) (1993) 917-932. http://www.jetp.ac.ru/cgi-bin/e/index/e/77/6/p917?a=list.

[215] H. J. de Vega and F. A. Schaposnik, "Electrically Charged Vortices in Nonabelian Gauge Theories With Chern-simons Term", Phys. Rev. Lett. 56 (1986) 2564. 
[216] C. N. Kumar and A. Khare, "Charged Vortex of Finite Energy in Nonabelian Gauge Theories With Chern-simons Term", Phys. Lett. B178 (1986) 395.

[217] J. L. Blázquez-Salcedo, L. M. González-Romero, F. Navarro-Lérida, and D. H. Tchrakian, "Non-Abelian Chern-Simons-Higgs vortices with a quartic potential", Phys. Rev. D88 no. 2, (2013) 025026. arXiv: 1306.5146 .

[218] F. Navarro-Lerida and D. H. Tchrakian, "Non-Abelian Yang-Mills-Higgs vortices", Phys. Rev. D81 (2010) 127702, arXiv:0909.4220.

[219] V. Markov, A. Marshakov, and A. Yung, "Non-Abelian Vortices in N = 1 * Gauge Theory", Nucl. Phys. B709 (2005) 267-295, arXiv:hep-th/0408235.

[220] R. Auzzi and S. P. Kumar, "Non-Abelian k-Vortex Dynamics in N=1* theory and its Gravity Dual", JHEP 12 (2008) 077, arXiv :0810.3201.

[221] P. Castorina, G. Riccobene, and D. Zappala, "Non-commutative dynamics and roton-like spectra in bosonic and fermionic condensates", Phys. Lett. A337 (2005) 463-468, arXiv:hep-th/0405093.

[222] S. Hands, "Four Fermion Models at Non-Zero Density", Nucl. Phys. A642 (1998) 228-238, arXiv:hep-lat/9806022.

[223] S. Choudhury, A. Dey, I. Halder, S. Jain, L. Janagal, S. Minwalla, and N. Prabhakar, "Bose-Fermi Chern-Simons Dualities in the Higgsed Phase", JHEP 11 (2018) 177, arXiv : 1804.08635

[224] L. Landau, "Theory of the superfluidity of helium ii", Phys. Rev. 60 (Aug, 1941) 356-358. 